\title{
INTERPLAY BETWEEN ACCOUNTING AND Prudential Regulation*
}

\author{
Jeremy Bertomeu
}

\author{
Lucas Mahieux
}

July 14,2020

\author{
Haresh Sapra
}

\begin{abstract}
We construct a model in which accounting information and prudential regulation interact to affect banks' incentives to originate loans. Prudential regulators impose capital requirements on banks but cannot commit to ex-ante efficient interventions and respond instead to ex-post accounting information. We show that accounting measurement and capital requirements are complementary tools that affect the level and efficiency of credit decisions. Comparative statics link capital requirement, quality of accounting information, and regulatory intervention to credit market conditions. An application is to the current debate on the expected loss provisioning model recently adopted in the financial industry.
\end{abstract}

Keywords: Accounting Standards; Prudential Regulation; Capital Requirements; Loan Loss Provisioning.

JEL codes: G21, G28, M41, M48.

\footnotetext{
* Jeremy Bertomeu is from the Rady School of Management at the University of California San Diego. Lucas Mahieux is from the School of Economics and Management at Tilburg University. Haresh Sapra is from the University of Chicago Booth School of Business. Contact information: l.mahieux@tilburguniversity.edu. We thank Catherine Casamatta, Edwige Cheynel, Yiwei Dou, Henry Friedman (discussant), Kurt Gee, Jonathan Glover, Thomas Hemmer, Xu Jiang, Pierre Liang, Iván Marinovic, Jack Stecher (discussant) and Gaoqing Zhang for various insights that have helped shape our model, as well as seminar and conference participants at the 13th Workshop on Accounting and Economics at Skema (Paris), 2017 Chicago-Minnesota Theory Conference, 2020 Accounting and Economics Society Webinars, 30th Accounting Research Conference at Washington University, University of Houston, University of Minnesota, and University of Zurich. Mahieux gratefully acknowledges funding from the European Research Council (grant agreement No 669217) and Sapra gratefully acknowledges financial support from the University of Chicago Booth School of Business and the Brevan Howard Center at Imperial College London. A previous version of the paper circulated under the title "Accounting versus Prudential Regulation." Any remaining errors are ours.
} 


\section{Introduction}

Prudential regulation relies on inputs from accounting numbers. However, in view of different institutional history and objectives, banking regulators and accounting standard setters do not implement policies based on an explicit articulation of joint mission statements. The mission of the Board of Governors of the Federal Reserve is to "foster the stability, integrity, and efficiency of the nation's monetary, financial, and payment systems so as to promote optimal macroeconomic performance" (Government Performance and Results Act Annual Performance Report, 2011) while standard setters such as the Financial Accounting Standard Board (FASB) aim at "providing financial information about the reporting entity that is useful to existing and potential investors" (FASB Concepts Statement No.8). In this paper, we provide a framework in which prudential regulators learn from banks' accounting systems. Such systems, in turn, depend on the prevailing accounting standards (Barth and Landsman 2010, Bushman 2014) and we highlight the role that accounting standards may play in influencing a bank's prudential regulation.

We augment a standard banking model in which a representative bank is subject to shareholders-depositors conflicts with a measurement friction. The bank's shareholders have incentives to engage in excessive risk-taking and the regulator can mitigate such inefficiency in two ways: (1) by imposing a capital requirement that constrains the ability of the bank's shareholders to originate risky loans and (2) by designing a reporting system informative about default risk and affecting the regulatory intervention policy. In order to provide efficient ex-ante incentives to originate safe loans, the regulator prefers an intervention policy that is excessive from an ex-post perspective - implying regulatory interventions in some otherwise healthy banks. However, this policy is not credible in a pure prudential regulation benchmark with full information on default risk. In response to full transparency, the prudential regulator must impose overly strict capital requirements, thereby constraining lending. 
The accounting reporting system can be designed ex-ante to increase ex-post regulatory interventions, similar to a conservative reporting system which makes bad news more likely. Excessive ex-post interventions, in turn, reduce the bank's risk-taking incentives and allows the regulator to set looser capital requirements. Our main result is that the optimal reporting system and capital requirement are complementary regulatory tools that generate more surplus when used together. Our model further allows us to examine how bank leverage and transparency adjust to shocks to the economic environment, and we discuss their dependence on the proportion of safe loans in the economy, the payoff structure of safe and risky loans, and the proceeds from regulatory interventions.

As an application, our model informs the recent debate surrounding the change in the way banks should recognize losses on their loan portfolios. Under the new standards (CECL and IFRS 9), banks no longer use an incurred loss model which has been criticized as delaying recognition of losses as it only considers current and historical information to determine if a credit loss exists. The new standards require banks to use an expected loss model to measure credit losses based on estimates of cash flows that the lender does not expect to collect. An expected loss incorporates historical information, current conditions, and reasonable forecasts of collection. By recognizing losses earlier, the threshold for recognizing losses would potentially be lower under an expected loss model relative to an incurred loss model. An insight from our model is that such excessive interventions induced by an expected loss model could indeed be socially desirable because it not only reduces the bank's risk-taking behavior but it also increases the bank's capacity to originate loans.

Literature review From a theoretical standpoint, there is an extensive literature showing how agency frictions place bounds on the size of firms, see, e.g., Holmstrom and Tirole (1997) or Liang, Rajan and Ray (2008). We borrow heavily from these ideas because in our model, a capital requirement on the bank bounds the size of its loan portfolio. To our knowledge, this literature does not jointly focus on the size of firms and the design of the 
information system which we view as the novel elements of our model.

The broader question of the optimal information design in response to agency problems has a long history in accounting, with contributions along two paths: first, the design of the information system can address commitment problems in contracting settings (Crémer 1995, Arya, Glover and Sivaramakrishnan 1997, Goex and Wagenhofer 2009); second, prior literature finds many environments in which reporting motives will affect investment (Kanodia and Sapra 2016, Gao and Jiang 2018). Our model borrows from both approaches, in that we examine the interactions between commitment, information design and the optimal size of a loan portfolio.

A strand of the banking and accounting literature has emerged since the 2007-08 financial crisis and highlights the differences between fair value and historical cost accounting. Bleck and Liu (2007), Allen and Carletti (2008), Plantin, Sapra and Shin (2008), Burkhardt and Strausz (2009), and Mahieux (2019) examine the impact of mark-to-market accounting on bank risk and financial stability. Heaton, Lucas and McDonald (2010) analyze the interaction between mark-to-market accounting and capital requirements in affecting the social cost of regulation. Corona, Nan and Zhang (2019a) examine the discretionary use of fair value accounting and its impact on bank lending. Lu, Sapra and Subramanian (2019) study the optimal use of mark-to-market accounting in implementing capital requirements, in the presence of asymmetric information and agency conflicts. Furthermore, Dewatripont and Tirole (1994) show that historical cost accounting may reduce the ability of prudential regulators to discipline banks, whereas Bleck and Gao (2017) study the effects of mark-to-market on banks' loan origination and retention decisions. While these preceding studies take the bank's information system as given or compare two accounting regimes, we endogenize the bank's accounting system as well as the prudential regulation.

Our paper is also related to the burgeoning theoretical literature that examines the role of accounting measurements and disclosure in affecting financial stability and prudential regulation, see Goldstein and Sapra (2014) for a recent survey. Corona, Nan and Zhang 
(2019b) examine the coordination role of stress-test disclosure in affecting bank risk-taking. Gao and Jiang (2018), Liang and Zhang (2019), and Zhang (2020) analyze the role of accounting information in stabilizing bank runs. Corona, Nan and Zhang (2015) examine the impact of accounting information quality on the efficiency of capital requirements and banks' risk-taking incentives, taking into account the competition among banks. In this paper, we solve for the optimal information system and we shed light on how regulatory capital should be tailored to the accounting standards. Our results therefore call for a better coordination between prudential regulators and accounting standard setters.

Within this literature, several studies are closely related to ours. Bleck and Opp (2019) focuses on optimal risk-based capital requirements when banks have private information about their desirable level of risk-taking and bank defaults cause a negative aggregate externality in the banking sector. In their model, the optimal regulatory mechanism with asymmetric information may feature bunching banks into separate risk classes. Li (2017) analyzes risk-taking incentives in banks in presence of capital regulation under different accounting regimes. She shows that the accounting regime that maximizes the social welfare is determined by a tradeoff between the social cost of capital regulation and the efficiency of the bank's project discovery efforts. Mahieux, Sapra and Zhang (2020) study two loan loss provisioning regimes and analyze the impact of early loss recognitions on banks' risk-taking incentives. In comparison to these studies, here, our focus is on undesirable risks and the regulator prefers to induce the bank to originate low-risk loan portfolios; further, we focus on excessive risk-taking due to the combination of banks' limited liability and deposit insurance: these assumptions imply that the losses from risky banks are not fully paid by banks and the depositors, requiring intervention to re-align social and private risk-taking incentives.

Several empirical studies provide evidence that financial reporting affects banks' risktaking incentives. Chircop and Novotny-Farkas (2016) suggest that extending the use of fair values for regulatory purposes reduces ex-ante risk-taking and Ellul, Jotikasthira, 
Lundblad and Wang (2015) find that the use of fair values in statutory accounting reduces ex-ante risk-taking incentives in insurance firms. Beatty and Liao (2011) and Bushman and Williams (2012) find that forward-looking provisions reflecting timely recognition of expected future loan losses is associated with enhanced risk-taking discipline. Consistent with these studies, we show how a well-designed accounting system may interact with a bank's capital requirement to control the bank's risk-taking incentives.

The rest of the paper proceeds as follows. Section 2 describes the baseline model. In section 3, we analyze the equilibrium with regulatory commitment, in the single policy benchmarks, and in the main model with joint policies respectively. We analyze the robustness of our results to alternative mechanisms in section 4 . In section 5 , we discuss the insights of our model with respect to the optimal loan loss provisioning model for banks. Finally, section 6 concludes. All the proofs are relegated to the appendix 1.

\section{The model}

\subsection{Timing of events}

We augment a standard risk-shifting banking model with a measurement friction. The model has four dates, indexed by $t=0, .5,1,2$, and features a regulator, a bank, and passive insured depositors. For simplicity, we assume that the bank's manager acts in the best interest of the bank's shareholders: hence, we use the terms "bank shareholders" and "bank" interchangeably in the rest of the paper. The regulator's task is to provide deposit insurance while maximizing social welfare, i.e., the expected surplus of the bank's loan portfolio. ${ }^{1}$ Figure 1 summarizes the sequence of events.

\footnotetext{
${ }^{1}$ The assumption that the regulator maximizes social welfare is consistent with the prior literature on banking regulation (Campbell, Chan and Marino 1992, Giammarino, Lewis and Sappington 1993, Hellmann, Murdock and Stiglitz 2000, Repullo 2004, Gorton and Winton 2017). Moreover, in line with a vast strand of the banking literature, we assume that deposits are insured for reasons outside the model (Hellmann et al. 2000, Repullo 2004, Boyd and De Nicolo 2005, Martinez-Miera and Repullo 2010). Note that we have left aside questions pertaining to runs, noting that there exists a literature modeling the issue of excessive coordination on accounting outputs, see, e.g., Plantin et al. (2008) or, recently, Gao
} 


\begin{tabular}{|c|c|c|c|}
\hline$t=0$ & $t=.5$ & $t=1$ & $t=2$ \\
\hline $\begin{array}{l}\text { The regulator chooses a } \\
\text { capital requirement } \gamma \\
\text { and a reporting system } \delta \text {. } \\
\text { Banks raise } D= \\
A-E \text { from depositors } \\
\text { s.t. } A / E \leq \gamma\end{array}$ & $\begin{array}{l}\text { The bank makes a risk } \\
\text { choice } r \text {. The bank then } \\
\text { originates a loan portfolio } \\
\text { and gets a safe loan or } \\
\text { a risky loan. }\end{array}$ & $\begin{array}{l}\text { The reporting system yields } \\
s \text { and reports assets } A_{1} \text {. } \\
\text { The bank violates the capital } \\
\text { requirement if } E_{1} / A_{1}<1 / \gamma, \\
\text { in which case the regulator } \\
\text { intervenes and may } \\
\text { restructure the loan. }\end{array}$ & $\begin{array}{l}\text { The loan payoff realizes: } \\
\alpha \text { for a safe loan, } \beta \\
\text { with prob. } p \text { and } 0 \text { with } \\
\text { prob. } 1-p \text { for a risky } \\
\text { loan without restructuring, } \\
\text { and } \mathcal{L} \text { for a risky } \\
\text { loan with restructuring. }\end{array}$ \\
\hline
\end{tabular}

Figure 1: Model timeline

At $t=0$, the bank has an exogenous amount of equity $E>0$. The regulator chooses a capital requirement $\gamma \geq 1$ for the bank, which we model as a maximum size of the bank's loan portfolio $A \geq E .^{2}$ The regulator also designs a reporting system $\delta$ that maps a random variable $p \in[0,1]$, capturing the probability of default of the bank's loan portfolio, to an interim signal $s \in[0,1]$ that will determine whether the regulator intervenes at $t=2$. The (inverse) probability of default $p$ has a distribution $F($.$) and a density f($.$) , mean p_{m}$ and full support on $[0,1]$.

Given the capital requirement $\gamma$ set by the regulator, the bank chooses its size $A$ and obtains $D=A-E$ from depositors. Deposits are fully insured and depositors receive a risk-free interest rate normalized to zero. At $t=0$, after raising deposits, the bank's balance sheet is therefore given by

$$
A=E+D \text { such that } \frac{A}{E} \leq \gamma
$$

At $t=.5$, the bank makes an unobservable risk choice $r \in\{0,1\}$ before originating a loan portfolio. Conditional on originating a low-risk loan portfolio $r=0$, the bank and Jiang (2018). Runs create an additional channel through which bank failures may contaminate other banks.

${ }^{2}$ The regulator only uses a simple leverage ratio to regulate capital. This is consistent with the newly proposed Basel III framework.

Under Basel II and III, bank regulators may also use some risk-weighted measure of assets to regulate capital. Adding an additional risk-weighted capital constraint in our model would not affect any of our results for two reasons. First, at $t=0$, the bank always satisfies the risk-weighted capital requirement because, prior to originating its loans, the bank's assets are consisted of only cash, which receives a risk weight of 0 . Second, as shown in our later analysis, with only the leverage requirement, the regulator already implements the ex-post optimal restructuring policy at $t=1$. 
receives a safe loan with probability $q \in(0,1)$ and a risky loan with probability $1-q$. Conditional on originating a high-risk loan portfolio $r=1$, the bank receives a risky loan with probability 1 . To avoid a trivial solution to the model, we assume that a high-risk portfolio is always socially undesirable while a low-risk portfolio has positive social value (see the formal assumptions at the end of this section). After making the risk choice $r$, the bank originates and invests its cash $A$ in the loan portfolio. ${ }^{3}$

We use a standard payoff structure for the safe loan and the risky loan to capture risktaking in the banking industry (Hellmann et al. 2000, Repullo 2004). A safe loan returns a payoff $\alpha$ regardless of the probability of default $p$, whereas a risky loan returns $\beta>\alpha$ with probability $p$ and zero with probability $1-p$. As a result, an increase in risk implies a larger payoff in case of no default but a higher likelihood of default. As in Hellmann et al. (2000) and Repullo (2004), this payoff structure combined with deposit insurance implies a negative risk externality: the bank and depositors do not bear the full loss from a failing loan because the bank's shareholders cannot lose more than the initial equity $E$ and depositors are fully insured. Hence, in the absence of capital requirements, the bank may increase its expected payoff by originating a value-destroying high-risk loan portfolio and does not internalize the cost on regulators from failing loans. In our model, therefore, capital requirements are useful because the regulator acts as a lender of last resort and bears the externality of failing loans. ${ }^{4}$

At $t=1$, the regulator and the bank observe a signal $s$ sent by the reporting system. The signal $s$ is equal to the true probability of default $p$ with probability $\delta \in[0,1]$ and is

\footnotetext{
${ }^{3}$ Note that, after originating the loan portfolio, whether the bank learns that the loan is safe or risky is irrelevant because the bank does not take any action after $t=.5$ in our model.

${ }^{4}$ The cost of failing loans in terms of making up the losses of depositors is only one of the potential costs but there could be other costs of risk-taking that would further increase the need (in our model) for regulation, see, e.g., Bleck (2018). In our model, prudential regulation is caused by the regulator acting as a lender of last resort: we study in section 4.2 an extension of our model in which there an additional negative externality in case of banking failure. One potential solution to this risk-shifting problem would be to prohibit loans whose interest rate is too high, which we do not allow in our model since we assume that loan characteristics are not contractible. However, in practice, not all loan characteristics are easily observable by regulators and issuing a set of acceptable interest rates conditional on each type of loan would require a degree of regulatory control that is far beyond current institutions.
} 
pure noise with probability $1-\delta$, in which case $s$ is randomly drawn from a distribution with density $f(.) .^{5}$ The parameter $\delta$ captures the precision of the reporting system designed by the regulator at $t=0 .{ }^{6}$

After observing the signal $s$, the bank updates the book value of its loan portfolio. The expected probability of default given the signal $s$ is

$$
\mathbb{E}(p \mid s)=\delta s+(1-\delta) p_{m}
$$

For the reporting system to affect the capital requirement, we assume that the reporting system delivers a measure of the bank's assets equal to the expected repayment of the loan

$$
A_{1} \equiv A(q \alpha+(1-q) \mathbb{E}(p \mid s) \beta)
$$

Given that the liability $D$ to depositors is fixed, the bank's equity is

$$
E_{1} \equiv A_{1}-D=A(q \alpha+(1-q) \mathbb{E}(p \mid s) \beta)-D
$$

As a result, at $t=1$, the bank violates the capital requirement $\gamma$ if and only if

$$
\frac{E_{1}}{A_{1}}<\frac{1}{\gamma}
$$

in which case the regulator intervenes. In practice, a regulator has broad discretion in terms of what actions to take upon intervention ranging from being passive, thereby allowing the bank to continue its operations to a reorganization, a partial asset sale, a reduction in the scope of the bank or even a liquidation. For simplicity, we focus on two possible regulatory

\footnotetext{
${ }^{5}$ Without loss of generality, specifying in these models the distribution of the noise to be equal to the distribution of the probability of default makes the analysis more tractable in that posterior expectations remain linear in the signal (Guttman and Marinovic 2018, Ganuza and Penalva 2019).

${ }^{6}$ The regulator only controls the probability that the interim signal $s$ is noisy but takes the distribution of the noise as a given. In section 4.3, we assume that the regulator controls the entire distribution of the accounting signal, i.e., we solve for the optimal persuasion mechanism, and we obtain similar results.
} 
actions: continuation or restructuring. We assume the regulator learns the type of the loan upon violation of the capital requirement. Otherwise, if the bank does not violate the capital requirement $\gamma$, the regulator does not intervene and there is continuation.

In our model, restructuring is not socially contractible, that is, for various reasons, the regulator cannot implement a policy to restructure the bank's loan if doing so does not increase surplus at $t=1 .^{7}$ Therefore, if the bank violates the capital requirement, the regulator restructures the bank's loan if and only if the expected payoff from restructuring is greater than the expected payoff from continuation.

We assume that a safe loan yields a payoff less than $\alpha$ with restructuring, so that the regulator always continues after observing a safe loan, in which case the bank gets the same payoff as without regulatory intervention. By contrast, if the bank has a risky loan and the regulator restructures the loan, the bank's shareholders do not recover terminal dividends. This assumption is consistent with most bank liquidations observed in practice (Granja, Matvos and Seru 2017). It can be micro-founded if banks cannot efficiently restructure loans and, instead, restructuring requires action by a second-best party such as a regulator or a better-capitalized intermediary. Only the regulator can restructure a risky loan and recover, possibly over time, a payoff $\mathcal{L} \in\left(p_{m} \beta, 1\right)$, so that the residual payoff of the bank conditional on restructuring is zero. ${ }^{8}$ The payoff of the risky loan $\mathcal{L}$ with restructuring is sufficiently large in order to rule out a setting in which restructuring would never be credible. This is consistent with Dewatripont and Tirole (1994) in which regulatory intervention is ex-post desirable if and only if the bank's asset is a low-quality asset.

\footnotetext{
${ }^{7}$ For most of the paper we shall maintain the assumption that the regulator cannot commit to a particular regulatory action before receiving the signal $s$. While the law may prescribe a capital requirement, law-makers might not ex-ante control what interventions a regulator would implement ex-post or may be subject to public pressure not to take corrective actions that reduce surplus ex-post. In section 3, we derive the results of our model when the regulator has full commitment power and we discuss why we believe that the no-commitment assumption is more appropriate.

${ }^{8}$ In appendix 2, we show that similar results hold even if the bank with a risky loan has some residual equity left after restructuring.
} 
At $t=2$, the payoff of the loan is realized. The payoff of the loan is $\pi=\alpha$ if the loan is safe; otherwise, if the loan is risky, the payoff is $\pi=\mathcal{L}$ with restructuring, and $\pi=\beta$ with probability $p$ or $\pi=0$ with probability $1-p$ in the absence of restructuring. The regulator compensates depositors if the bank fails, i.e., if $A \pi<A-E$, with a lump sum payment which we assume is financed via an ex-ante tax.

\subsection{Assumptions}

For our baseline model, we make the following assumptions 1-4 on the parameter values.

Assumption 1. If the bank originates a high-risk loan portfolio $(r=1)$, the expected surplus is negative:

$$
\mathbb{E}(\max (\mathcal{L}, p \beta))<1
$$

This assumption ensures that regulator must provide incentives to the bank to originate a low-risk loan portfolio to create value.

Assumption 2. If the bank size is arbitrarily large, the bank's loan portfolio has a negative NPV. A sufficient condition for this to hold is that, at the minimal precision $\delta_{0}$ such that the NPV of the low-risk loan portfolio is zero, the bank's incentive constraint does not hold for an arbitrarily large bank size:

$$
\int_{\frac{1}{\delta_{0}}\left(\frac{\mathcal{L}}{\beta}-\left(1-\delta_{0}\right) p_{m}\right)}^{1}\left(\delta_{0} p+\left(1-\delta_{0}\right) p_{m}\right) f(p) d p>\frac{\alpha-1}{\beta-1} \cdot 9
$$

The precision $\delta_{0}$ can be explicitly written as

$$
q \alpha+(1-q) \mathbb{E}\left(\max \left(\mathcal{L},\left(\delta_{0} p+\left(1-\delta_{0}\right) p_{m}\right) \beta\right)\right)=1
$$

\footnotetext{
${ }^{9}$ In Lemmas 1-4, we will prove that Equation (2.5) is the bank's incentive constraint for an arbitrarily large $A$ evaluated at the restructuring policy $\frac{1}{\delta_{0}}\left(\frac{\mathcal{L}}{\beta}-\left(1-\delta_{0}\right) p_{m}\right)$ associated to precision $\delta_{0}$.
} 
Assumption 2 also implies that, if the bank size is arbitrarily large and the regulator learns $p$ perfectly, the bank originates a high-risk loan portfolio. ${ }^{10}$

Assumption 3. A low-risk loan portfolio has a positive value if the reporting system is perfectly informative:

$$
q \alpha+(1-q) \mathbb{E}(\max (\mathcal{L}, p \beta))>1
$$

Assumption 3 rules out a corner solution in which both the high-risk loan portfolio and the low-risk loan portfolio are value-destroying. Note that assumption 3 together with assumption 1 imply that $\beta>\alpha>1>\mathcal{L}$.

Assumption 4. The bank is not sufficiently capitalized:

$$
\frac{(1-q)(\alpha-\mathcal{L})}{\beta-q \alpha-(1-q) \mathcal{L}}>\int_{\frac{\mathcal{L}}{\beta}}^{1} p f(p) d p .
$$

This assumption guarantees that the bank violates the capital requirement at $t=1$ when it is optimal for the regulator to restructure a risky loan. ${ }^{11}$

\section{Main analysis}

We solve the model by backward induction. To this end, we start by solving the regulator's optimal restructuring decision at $t=1$ given the signal $s$ sent by the reporting system. Next, we solve for the risk decision $r$ of the bank and the bank's choice of $A$. Lastly, we solve for the optimal reporting system $\delta$ and the optimal capital requirement $\gamma$ set by the regulator at $t=0$.

We will show that the bank optimally chooses $A^{*}$ such that the capital requirement $\gamma$

\footnotetext{
${ }^{10}$ Formally, assumption 2 implies that $\frac{\alpha-1}{\beta-1}<\int_{\frac{\mathcal{L}}{\beta}}^{1} p f(p) d p$.

${ }^{11}$ If this is condition is not satisfied, the regulator would have to set a second interim capital requirement in order to implement the ex-post optimal restructuring policy at $t=1$. The results are unchanged in this extended setting.
} 
is binding at $t=0$. Therefore, at $t=1$, given the signal $s$ sent by the reporting system, the equity of the bank is

$$
E_{1}=A(q \alpha+(1-q) \mathbb{E}(p \mid s) \beta)-D
$$

If $q \alpha+(1-q) \mathbb{E}(p \mid s) \beta \geq 1$, the bank does not violate the capital requirement $\gamma$ :

$$
\frac{E_{1}}{A_{1}}=\frac{A(q \alpha+(1-q) \mathbb{E}(p \mid s) \beta)-D}{A(q \alpha+(1-q) \mathbb{E}(p \mid s) \beta)} \geq \frac{E}{A}=\frac{1}{\gamma}
$$

and the regulator does not restructure the loan. Otherwise, if $q \alpha+(1-q) \mathbb{E}(p \mid s) \beta<1$, the bank violates the capital requirement $\gamma$ :

$$
\frac{E_{1}}{A_{1}}<\frac{1}{\gamma}
$$

The regulator then restructures a risky loan if and only if the associated payoff is greater than the expected continuation value, i.e., $q \alpha+(1-q) \mathbb{E}(p \mid s) \beta<q \alpha+(1-q) \mathcal{L}$, which we can write in terms of the bank equity:

$$
E_{1}<\hat{E} \equiv A(q \alpha+(1-q) \mathcal{L})-D
$$

The following lemma summarizes the policy implemented by the regulator in this model.

Lemma 1 At $t=1$, conditional on realized equity $E_{1}$, (i) if $E_{1} / A_{1}>1 / \gamma$, the bank does not violate the capital requirement and the regulator does not restructure the loan; (ii) if $E_{1} / A_{1} \in\left[\hat{E} / A_{1}, 1 / \gamma\right)$, the bank violates the capital requirement but the regulator does not restructure the loan; and (iii) if $E_{1} / A_{1}<\hat{E} / A_{1}$, the bank violates the capital requirement and the regulator restructures the loan if this is a risky loan.

The regulator first observes the level of equity of the bank $E_{1}$ after the accounting signal $s$ is received, and two scenarios may then occur. If the bank does not violate 
the capital requirement $(i)$ or restructuring would not increase value $(i i)$, the regulator anticipates that restructuring would decrease social value at this point of the game. On the other hand, if the bank's equity $E_{1} \leq \hat{E}$ is sufficiently low, the bank violates the capital requirement and the regulator restructures the loan if the regulator identifies a risky loan.

Given the realized signal $s$ sent by the reporting system at $t=1$, it is convenient to rewrite the restructuring decision in (3.2) in terms of a threshold $\tau(\delta)$ such that the regulator restructures a risky loan if and only if $p<\tau(\delta)$, where $\tau(\delta)$ is defined as follows

$$
\tau(\delta) \equiv \begin{cases}1 & \text { if } 0 \leq \delta<\frac{\frac{\mathcal{L}}{\beta}-p_{m}}{1-p_{m}} \\ \frac{1}{\delta}\left(\frac{\mathcal{L}}{\beta}-(1-\delta) p_{m}\right) & \text { otherwise }\end{cases}
$$

Figure 2, below, plots the bank's equity $E_{1}$ as a function of the (inverse) probability of default against the restructuring threshold: the regulator restructures a risky loan when $E_{1} \leq \hat{E}$, to the left of the intersection between the horizontal line and the upward sloping continuation payoff. ${ }^{12}$ The continuation payoff is steepest in the probability of default when the reporting system is perfect $(\delta=1)$. As the reporting system becomes more imprecise (lower $\delta$ ), the continuation payoff rotates, increasing the set of reports such that the regulator restructures a risky loan. In other words, by creating uncertainty about the probability of default, an imprecise measure increases the likelihood of ex-post restructuring. The next lemma summarizes this result and provides additional comparative statics.

Lemma 2 The restructuring threshold $\tau(\delta)$ increases in the restructuring payoff $\mathcal{L}$, and decreases in the payoff of a risky loan $\beta$ and in the precision $\delta$ of the reporting system.

Next, we derive the bank's expected payoff and examine the bank's choice of $A$ as well as the bank's risk decision $r$. Recall that the bank with a risky loan receives a zero payoff with restructuring but, otherwise, receives a payoff $A \alpha-(A-E)$ when continuing with a

\footnotetext{
${ }^{12}$ This was evaluated at $q=0.7, \alpha=1.1, \mathcal{L}=.45, p_{m}=.2, D=0.3, A=1$ and $\beta=1.2$.
} 


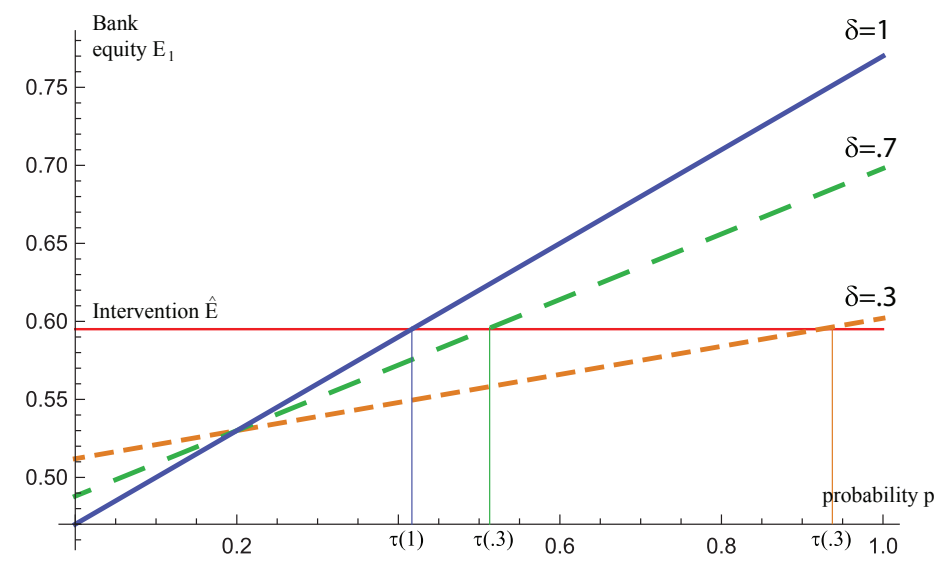

Figure 2: Restructuring policy as a function of the probability of default

safe loan and $\mathbb{E}(p \mid s)(A \beta-(A-E))$ when continuing with a risky loan. In the next lemma, we show that the expected payoff of the bank increases with bank size, which implies that the capital requirement $\gamma$ is binding at $t=0$.

Lemma 3 Conditional on originating a loan portfolio, the bank's expected payoff is increasing in the bank size A for any $\delta$. Hence, for a given capital requirement $\gamma$, the bank chooses $A^{*}=\gamma E$.

With a perfectly informative reporting system $\delta=1$ and no capital requirement, the bank would choose the maximal bank size and would originate a high-risk loan portfolio (assumption 2), which is suboptimal for the regulator (assumption 1). This result highlights the role of capital requirements in our setting. We capture one of the two key market failures in the banking industry: insured depositors do not monitor the management of their banks and, as a result, banks are engaged in excessive risk-taking (Hellmann et al. 2000, Repullo 2004, Freixas and Rochet 2008). Hence, the regulator needs to address the risk-shifting with a capital requirement and an accounting reporting system.

We state next the bank's incentive constraint. At $t=.5$, the bank prefers to originate a low-risk loan portfolio, i.e, $r=0$, over a high-risk loan portfolio, i.e., $r=1$, if and only 
if

$$
\underbrace{q(A \alpha-(A-E))+(1-q) k(\delta)(A \beta-(A-E))}_{\text {bank's expected payoff with } r=0} \geq \underbrace{k(\delta)(A \beta-(A-E))}_{\text {bank's expected payoff with } r=1}
$$

where

$$
k(\delta)=\int_{\tau(\delta)}^{1}\left(\delta p+(1-\delta) p_{m}\right) f(p) d p
$$

captures the expected value of the (inverse) probability of default times the probability that the regulator does not restructure a risky loan. This incentive constraint is equivalent to:

$$
(A \alpha-(A-E)) \geq k(\delta)(A \beta-(A-E))
$$

Given that the bank chooses the maximal bank size such that $A^{*}=\gamma E$, the incentive constraint (3.5) can be conveniently expressed in terms of a constraint on the capital requirement

$$
\gamma \leq \frac{1-k(\delta)}{k(\delta)(\beta-1)-(\alpha-1)}
$$

In summary, at $t=0$, the regulator's problem is to maximize the expected surplus from the bank's loan by inducing the bank to originate a low-risk loan portfolio. The maximization problem of the regulator can be written as

$$
\max _{\gamma, \delta} \Sigma(\gamma, \delta)=(q \alpha+(1-q)(F(\tau(\delta)) \mathcal{L}+k(\delta) \beta)-1) \gamma E
$$

s.t.

$$
\gamma \leq \frac{1-k(\delta)}{k(\delta)(\beta-1)-(\alpha-1)}
$$

Before solving this maximization problem, note that the expected surplus from the bank's loan is increasing in the bank size $A$, or, equivalently, in the capital requirement $\gamma$. As a result, the regulator finds it optimal to set the capital requirement $\gamma$ such that the incentive constraint of the bank always binds in equilibrium. 
Lemma 4 The risk-shifting incentive constraint of the bank (3.7) binds in equilibrium.

Lemma 4 implies that, for the incentive constraint (3.7) can be rewritten as

$$
\gamma=\frac{1-k(\delta)}{k(\delta)(\beta-1)-(\alpha-1)}
$$

Intuitively, the greater the payoff from a risky loan $\beta$ (resp. safe loan $\alpha$ ), the more attractive to the bank such loans are, the stricter (resp. looser) the capital requirement. Further, from (3.4), the function $k(\delta)$ decreases with the restructuring threshold $\tau(\delta)$. As a result, a higher likelihood of restructuring facilitates a looser capital requirement, thus revealing the economic channel through which the reporting system and the capital requirement interact. We formally state and prove those comparative statics in the single policy benchmarks section below. Before that, we explain the importance of the lack of regulatory commitment power in this environment.

Regulatory commitment. Lack of commitment power to efficiently restructure is a critical part of the role of information in our model, similar to Crémer (1995) or Arya, Glover and Sivaramakrishnan (1997). This assumption is consistent with the practice of prudential regulation and has been examined extensively in the literature (Dewatripont and Tirole 1994). ${ }^{13}$ In our setting, the regulator choosing to act at $t=1$ does not internalize the impact of restructuring on the bank's risk decision because this decision is sunk by the time restructuring occurs. Below, we develop this point further by solving program $(P)$ with regulatory commitment, that is, replacing the restructuring threshold $\tau(\delta)$ by a

\footnotetext{
${ }^{13}$ It is also consistent with a wide literature on dynamic inconsistency in policy-making starting with Kydland and Prescott (1977). Many prior studies build on this assumption, noting that this type of inconsistency arises because (banking) regulators cannot ignore political pressures (Dewatripont and Tirole 1994, Rochet 2004). Dewatripont and Tirole (1994) also argue that regulatory capture may be a reason for the lack of commitment power of banking regulators. Relatedly, Boot and Thakor (1993) show that, if regulators are self-interested, they may be captured by the banking industry. Finally, when the time comes to enforce their commitment, prudential regulators often ignore long-term concerns and violate earlier commitment because of the "too-big-to-fail" or "too-many-to-fail" issues (Stern and Feldman 2004, Acharya and Yorulmazer 2007)
} 
restructuring threshold $\tau_{c}$ independent of the reporting system, and optimizing in $\left(\gamma, \delta, \tau_{c}\right)$.

Proposition 1 With commitment power, the regulator always sets $\delta_{c}^{*}=1$ and restructures a risky loan if and only if $p<\tau_{c}^{*}$, where the threshold $\tau_{c}^{*}$ is such that $\tau_{c}^{*}>\tau(1)=\frac{\mathcal{L}}{\beta}$, and the regulator imposes a capital requirement

$$
\gamma_{c}^{*}=\frac{1-\int_{\tau_{c}^{*}}^{1} p f(p) d p}{\int_{\tau_{c}^{*}}^{1} p f(p) d p(\beta-1)-(\alpha-1)} .
$$

With full commitment power, the regulator is better-off using as much information as possible to condition restructuring on high probabilities of default (low $p$ ), implying a perfectly informative reporting system $\delta_{c}^{*}=1$. But we also show that the restructuring threshold $\tau_{c}^{*}$ is stricter than the threshold that would prevail absent commitment: for moderate realizations of the probability of default $p \in\left(\tau(1), \tau_{c}^{*}\right)$, a regulator would be ex-post better-off not restructuring. As a result, excessive restructurings are part of the optimal regulation because the excessive restructurings curb the bank's ex-ante risk-taking incentives, which allows the regulator to set a looser capital requirement. In what follows, we show that, without commitment power, excessive restructurings can be achieved via an imprecise reporting system.

Single policy benchmarks. To analyze the interaction between the two regulatory tools, we first examine below the comparative statics of two benchmarks in which the regulator optimizes only one regulatory tool. First, in the prudential benchmark, the precision of the reporting system $\delta=\delta_{f}$ is given and the regulator sets the optimal capital requirement $\gamma^{*}$. Recall that the precision $\delta_{0}$ is such that the expected surplus from a low-risk loan portfolio is 0 :

$$
q \alpha+(1-q)\left(F\left(\tau\left(\delta_{0}\right)\right) \mathcal{L}+k\left(\delta_{0}\right) \beta\right)=1
$$


As a result, if $\delta_{f}<\delta_{0}$, the regulator sets $\gamma^{*}=1$, and the bank does not originate a loan portfolio because the expected surplus from both loan portfolios is negative. Otherwise, if $\delta_{f} \geq \delta_{0}$, the regulator sets $\gamma^{*}$ such that the bank's incentive constraint is binding.

Second, in the measurement benchmark, the capital requirement $\gamma=\gamma_{f}$ is fixed and the regulator designs the optimal reporting system $\delta^{*}$. If $\gamma_{f} \leq \frac{1-k(1)}{k(1)(\beta-1)-(\alpha-1)}$, then the capital requirement is sufficiently tight so that the bank always chooses to originate a low-risk loan portfolio whatever the precision of the reporting system. The regulator therefore sets $\delta^{*}=1$ to maximize the expected surplus. Otherwise, if $\gamma_{f}>\frac{1-k(1)}{k(1)(\beta-1)-(\alpha-1)}$, the regulator has to control the bank's risk-taking incentives with the reporting system. The regulator designs the reporting system $\delta^{*}$ such that the bank's incentive constraint is binding. Note that, if $\gamma_{f}>\frac{1-k\left(\delta_{0}\right)}{k\left(\delta_{0}\right)(\beta-1)-(\alpha-1)}$, the surplus is negative as the capital requirement is excessively loose, which implies that the bank's risk-shifting problem is too severe. Proposition 2 summarizes the equilibrium in the single policy benchmarks.

Proposition 2 First, in the prudential benchmark, if $\delta_{f}<\delta_{0}$, the regulator sets $\gamma^{*}=1$. Otherwise, if $\delta_{f} \geq \delta_{0}$, the optimal capital requirement is given by

$$
\gamma^{*}=\frac{1-k\left(\delta_{f}\right)}{k\left(\delta_{f}\right)(\beta-1)-(\alpha-1)}
$$

and is decreasing in the precision $\delta_{f}$ of the reporting system.

Second, in the measurement benchmark, if $\gamma_{f} \leq \frac{1-k(1)}{k(1)(\beta-1)-(\alpha-1)}$, then the optimal precision of the reporting system is $\delta^{*}=1$; otherwise, if $\gamma_{f}>\frac{1-k(1)}{k(1)(\beta-1)-(\alpha-1)}$, the optimal precision $\delta^{*}$ is defined by $k\left(\delta^{*}\right)=\frac{1+(\alpha-1) \gamma_{f}}{1+(\beta-1) \gamma_{f}}$ and is decreasing in the capital requirement $\gamma_{f}$.

With a single policy benchmark, the capital requirement and the reporting system are substitutes, as both serve to curb the bank's risk-taking incentives. This result is entirely intuitive and serves to set ideas for the intuitions in the model with two optimal policies. Specifically, proposition 2 implies that a reporting system inducing more restructurings 
mitigates the risk-taking problem and allows the regulator to run a larger bank. Viceversa, if the capital requirement is very strict, a perfectly informative reporting system would provide sufficient restructuring incentives to control the risk-taking problem but, otherwise, the reporting system must induce more restructurings with a larger capital ratio. In corollary 1 below, we derive the comparative statics of each of these policies for changes in the environment.

Corollary 1 In the single policy benchmarks, the optimal capital requirement $\gamma^{*}$ and the optimal precision $\delta^{*}$ increase in the payoff $\alpha$ of a safe loan, and in the restructuring payoff $\mathcal{L}$ of a risky loan, and decrease in the payoff $\beta$ of a risky loan.

In the two benchmarks, reducing bank size via a stricter capital requirement or inducing more ex-post restructurings via a less precise reporting system reduce the expected loan surplus, so how much to use the tools is a function of the attractiveness of the high-risk loan portfolio. As expected, the capital requirement is stricter and the reporting system more imprecise when the payoff $\alpha$ of a safe loan and the restructuring payoff $\mathcal{L}$ is lower, and when the payoff $\beta$ of a risky loan is higher. Importantly, a key take-away of corollary 1 is that both regulatory tools respond to an economic primitive of the model in the same direction.

Joint maximization problem. We next solve the general case in which the regulator jointly chooses the optimal capital requirement $\gamma$ and the optimal precision $\delta$ of the reporting system. To decompose this problem, it is convenient to rewrite the objective function of the regulator as

$$
\Sigma(\gamma, \delta) \equiv N P V(\delta) \phi(\delta) E,
$$

where $N P V(\delta) \equiv q \alpha+(1-q)(F(\tau(\delta)) \mathcal{L}+k(\delta) \beta)-1$ is the net present value of the lowrisk loan portfolio, $\phi(\delta)$ is the required capital requirement. Note that $\phi(\delta) \equiv \gamma$ given the precision $\delta$ to bind the bank's incentive constraint (3.7). Hence, the first-order condition 
of the optimization program $(P)$ with respect to $\delta$ can be written as

$$
\begin{aligned}
\left.\frac{d \Sigma(\phi(\delta), \delta)}{d \delta}\right|_{\delta=\delta^{*}} & =N P V^{\prime}\left(\delta^{*}\right) \phi\left(\delta^{*}\right) E+N P V\left(\delta^{*}\right) \phi^{\prime}\left(\delta^{*}\right) E \\
& =\underbrace{(1-q) \int_{\tau(\delta)}^{1}\left(p-p_{m}\right) f(p) d p \beta \phi\left(\delta^{*}\right) E}_{\text {benefit of increasing precision }}-\underbrace{\frac{N P V\left(\delta^{*}\right) k^{\prime}\left(\delta^{*}\right)(\beta-\alpha) E}{\left(k\left(\delta^{*}\right)(\beta-1)-(\alpha-1)\right)^{2}}}_{\text {cost of increasing precision }} \\
& =0 .
\end{aligned}
$$

The first-order condition is composed of two terms. The first term is the benefit of increasing precision and implies more valuable loans because restructuring more accurately target probabilities of default in which the regulator should restructure risky loans. The second term reflects how an increase in precision requires a stricter capital requirement and, hence, a smaller bank size. Contrasting with the single policy model in which the policy is held fixed by the incentive constraint, there is now a trade-off between increasing loan value via a looser capital requirement or a more informative reporting system. We examine this trade-off in the following proposition and the associated corollary below.

Proposition 3 The regulator sets the capital requirement $\gamma^{*}=\frac{1-k\left(\delta^{*}\right)}{k\left(\delta^{*}\right)(\beta-1)-(\alpha-1)}$ and the reporting system is not fully precise, i.e., $\delta^{*}<1$, if either (a) the payoff of a safe loan $\alpha$ is large enough, (b) the proportion of safe loans $q$ is large enough, or (c) the restructuring payoff $\mathcal{L}$ is large enough, $(\beta-1)+(\alpha-1)-2 k(1)(\beta-1) \geq 0$, and $f^{\prime}\left(\frac{\mathcal{L}}{\beta}\right)$ is positive or sufficiently close to 0.

Proposition 3 demonstrates that the regulator may use a combination of imprecise accounting information to induce the optimal level of restructuring, jointly with a capital requirement that is less strict than with a perfectly informative reporting system. On the one hand, imprecision triggers excessive restructurings for a given capital requirement and, therefore, allows for a looser capital requirement and a larger bank size. On the other 
hand, imprecision matches restructuring less precisely with the probability of default, and, therefore, reduces the $N P V$ of the low-risk loan portfolio.

The regulator optimally designs an imprecise information system when the risk-taking problem is not too severe under full information, i.e., if the payoff of a safe loan is large enough so that $k(1)(\beta-1)-(\alpha-1)$ is sufficiently small. In that case, the regulator controls the risk-taking problem using a less precise reporting system and allows the bank to choose a larger size. Similarly, the regulator finds it optimal to increase the size of the bank via a less precise reporting system when the proportion $q$ of safe loans is large, since this implies that each low-risk loan portfolio is ex-ante more valuable. Lastly, an increase in the restructuring payoff $\mathcal{L}$ may also lead to more imprecision. ${ }^{14}$ In summary, a policy with imprecision in the measurement and a looser capital requirement dominates a policy with full precision and a stricter capital requirement in environments where the risk-taking problem is not too severe and/or loans are more valuable.

Let us now study how the optimal reporting system $\delta^{*}$ and the optimal capital requirement $\gamma^{*}$ vary with the different parameters of our model. In order to derive the comparative statics, we make the (generic) assumption that the regulator's objective function $\Sigma(\gamma, \delta)$ has a unique global maximum, so that optimal policies change continuously in each parameters.

Corollary 2 The optimal precision of the reporting system $\delta^{*}$ (the capital requirement $\gamma^{*}$ ) decreases (increases) in (a) the payoff $\alpha$ of a safe loan, (b) the proportion of safe loans $q$, and $(c)$ the restructuring payoff $\mathcal{L}$ if $(\beta-1)+(\alpha-1)-2 k(1)(\beta-1) \geq 0$ and $f^{\prime}\left(\tau\left(\delta^{*}\right)\right)$ is positive or sufficiently close to $0 .{ }^{15}$ Further, for any parameter values such that $\partial \delta^{*} / \partial \beta>0$ or $\partial \gamma^{*} / \partial \beta>0, \delta^{*}$ and $\gamma^{*}$ vary in the opposite direction as a function of $\beta$.

\footnotetext{
${ }^{14}$ If $p$ is uniformly distributed, then the conditions in proposition 3 are satisfied: $f^{\prime}\left(\frac{\mathcal{L}}{\beta}\right)=0$ and $(\beta-1)+(\alpha-1)-2 k(1)(\beta-1)=\alpha-1+\left(\frac{\mathcal{L}}{\beta}\right)^{2}(\beta-1)>0$.

${ }^{15}$ This occurs when $\tau\left(\delta^{*}\right)$ is not too large in single-peaked distributions, which is the case when $\alpha$ is sufficiently low as $\delta^{*}$ decreases in $\alpha$ and $\tau(\delta)$ decreases in $\delta$.
} 
Corollary 2 shows how the comparative statics in the model using both an optimal capital requirement and the optimal level of precision may not be fully aligned with those with a single optimal policy. Recall that if the regulator uses a single optimal policy, the policy must (intuitively) respond to environments with more demanding credit frictions with stricter policies, e.g., a stricter capital requirement or a less precise reporting system that triggers more restructurings. Using the two optimal policies in tandem, however, reveals a more nuanced answer and implies that the capital requirement and imprecision of the reporting system often vary in opposite directions.

First, an increase in the payoff of a safe loan increases the payoff from larger banks and, while it also makes it easier to solve the risk-shifting problem because safe loans are more attractive to the bank, the adjustment takes the form of decreasing the precision of the reporting system - i.e., reallocate the provision of incentives toward the reporting system. Second, an increase in the proportion of safe loans increases the NPV of a low-risk loan portfolio, which makes bank size more attractive. The regulator induces a larger bank by increasing imprecision and loosening the capital requirement. Third, the effect of the restructuring payoff is more ambiguous but, if the density of probabilities of default does not decrease too steeply (as in the case of the uniform distribution), an increase in this payoff implies a decrease in precision because, similar to the other comparative statics, the regulator re-allocates the provision of risk-taking incentives toward the reporting system and tends to prefer a larger bank.

Lastly, the impact of $\beta$, the payoff of the risky loan, is the result of two opposing effects. First, an increase in $\beta$ makes a high-risk loan portfolio - which reduces expected surplus more attractive to the bank and aggravates the risk-taking problem. Second, an increase in $\beta$ increases the expected surplus from a high-risk loan portfolio. Therefore, even though a high-risk loan portfolio reduces social surplus under our maintained assumption 1, the adverse impact of a risky loan on the bank's surplus becomes more muted as $\beta$ increases. The latter effect increases the expected surplus generated by the bank. Corollary 2 reveals 


\begin{tabular}{l|l||cccc} 
& & $\alpha$ & $\mathcal{L}$ & $q$ & $\beta$ \\
\hline Joint maximization & $\gamma^{*}$ & + & + & + & $+/-$ \\
with $\delta^{*}<1$ & $\delta^{*}$ & - & - & - & $+/-$ \\
\hline $\begin{array}{l}\text { Exogenous precision } \\
\text { with } \delta_{f} \geq \delta_{0}\end{array}$ & $\gamma^{*}$ & + & + & 0 & - \\
\hline $\begin{array}{l}\text { Exogenous capital re- } \\
\text { quirement with } \gamma_{f}> \\
\begin{array}{l}1-k(1) \\
k(1)(\beta-1)-(\alpha-1)\end{array}\end{array}$ & $\delta^{*}$ & + & + & 0 & - \\
& & & & &
\end{tabular}

Table 1: Summary of the comparative statics

that, as for the other comparative statics, the regulatory tools may vary in the opposite direction as a function of $\beta$. In particular, if the regulator sets a looser capital requirement in response to higher $\beta$, restructurings must increase and the regulator chooses a less precise information system. Vice-versa, if the regulator increases the precision of the reporting system, then the more severe agency problem must be solved with a stricter capital requirement.

Table 1 summarizes the comparative statics of our baseline model. ${ }^{16}$ The main insight is that, in the single policy benchmarks, the capital requirement and the reporting system are substitutes and respond to an economic primitive of the model in the same direction. On the contrary, in the joint maximization problem, the capital requirement and the reporting system are complements and respond to an economic primitive of the model in the opposite direction.

\section{Robustness to other mechanisms}

\subsection{Adverse selection}

We now analyze a variant of our model in which the main friction is an adverse selection problem instead of a moral hazard problem. As noted by Chan, Greenbaum and Thakor

\footnotetext{
${ }^{16}$ Under the conditions of corollary 2 for the comparative statics with respect to $\mathcal{L}$.
} 
(1992), adverse selection and moral hazard problems are two key frictions that provide a rationale for banking regulation. In our baseline model, we derive our results assuming that the regulator faces a moral hazard problem. In this extension, we show that the optimal regulatory tools are similar when the regulator faces instead an adverse selection problem.

We first emphasize the differences in the model setup and then discuss the main results. The bank may be one of two types with equal probability: a good bank that always originates a low-risk loan portfolio or a bad bank that always originates a high-risk loan portfolio. The timeline is the same as in the baseline model. The only differences are the following. At $t=0$, the bank observes its type and may decide not to operate, in which case it consumes $E$ and the game ends. Otherwise, the bank chooses size $A$ and obtains $D=A-E$ from depositors. At $t=0.5$, the good bank originates a low-risk loan portfolio and receives a safe loan with probability $q \in(0,1)$ and a risky loan with probability $1-q$. The bad bank originates a high-risk loan portfolio and always gets a risky loan with probability $1 .{ }^{17}$

In order to induce a bad bank not to originate a high-risk loan portfolio, the following constraint must be met:

$$
\underbrace{E}_{\text {bad bank's payoff without origination }} \geq \underbrace{k(\delta)(A \beta-(A-E))}_{\text {bad bank's expected payoff with origination }} .
$$

As in our baseline model, equation (4.3) reveals how restructuring reduces incentives to

\footnotetext{
${ }^{17}$ The equivalent of assumption 2 in this alternative setup that rules out a corner solution with an arbitrarily large bank size is the following assumption. If the leverage of the bank is maximum $(A / E=\infty)$, the surplus is negative. A sufficient condition is

$$
q \alpha+(1-q)\left(F(\tau(\hat{\delta})) \mathcal{L}+\int_{\tau(\hat{\delta})}^{1}\left(\hat{\delta} p+(1-\hat{\delta}) p_{m}\right) f(p) d p \beta\right)<1
$$

where $\hat{\delta}$ is defined such that

$$
\int_{\tau(\hat{\delta})}^{1}\left(\hat{\delta} p+(1-\hat{\delta}) p_{m}\right) f(p) d p=0
$$

and the restructuring threshold $\tau(\delta)$ is the same as in our baseline model.
} 
originate a high-risk loan portfolio, by reducing the payoff receives by the bank over a wider set of probabilities of default. Lastly, the good bank must be better-off originating a loan portfolio over not originating a loan portfolio, i.e.,

$$
\underbrace{q(A \alpha-(A-E))+(1-q) k(\delta)(A \beta-(A-E))}_{\text {good bank's expected payoff with origination }}>\underbrace{E}_{\text {good bank's payoff without origination }} .
$$

Note that the assumption $\alpha>1$ implies that the participation constraint of the good bank (4.4) is always strictly satisfied when the participation constraint of the bad bank (4.3) binds. In summary, the objective of the regulator can be written as:

$$
(P) \quad \max _{\gamma, \delta} \Sigma(\gamma, \delta)=\frac{1}{2}(q \alpha+(1-q)(F(\tau(\delta)) \mathcal{L}+k(\delta) \beta)-1) \gamma E
$$

s.t.

$$
\gamma \leq \frac{1-k(\delta)}{k(\delta)(\beta-1)}
$$

Note that condition (4.5) is more demanding than eliciting effort in the baseline moral hazard model (3.8) because the denominator is $k(\delta)(\beta-1)$ instead of $k(\delta)(\beta-1)-(\alpha-1)$ in the baseline model. Hence, the regulatory actions must be stricter in the adverse selection model and, doing so, would be sufficient to provide incentives for the good bank to choose the low-risk loan portfolio. In other words, in an environment in which the regulator faces both an adverse selection problem and a moral hazard problem, solving the adverse selection problem would solve the moral hazard problem.

Proposition 4 The reporting system is not fully precise, i.e., $\delta^{*}<1$, if either (a) the payoff of a safe loan $\alpha$ is large enough, (b) the proportion of safe loans $q$ is large enough, or (c) the restructuring payoff $\mathcal{L}$ is large enough, $1-2 k(1) \geq 0$, and $f^{\prime}\left(\frac{\mathcal{L}}{\beta}\right)$ is positive or sufficiently close to 0 .

We also prove in the appendix 1 that the comparative statics in the adverse selection 
have the same nature as in the moral hazard environment, with the only primary difference being that the regulator needs to impose stricter regulations.

Lastly, we show that the regulatory policies $\left(\gamma^{*}, \delta^{*}\right)$ derived above are the optimal policies and the regulator cannot increase the expected surplus with two sets of policies targeted to good banks and bad banks. In particular, the regulator could set different capital requirements and different reporting systems $\left(\gamma_{G}, \delta_{G}\right)$ and $\left(\gamma_{B}, \delta_{B}\right)$ such that, in equilibrium, a good bank voluntary chooses the policies $\left(\gamma_{G}, \delta_{G}\right)$ whereas a bad bank chooses the policies $\left(\gamma_{B}, \delta_{B}\right)$. However, the following corollary states that the regulator cannot increase the expected surplus compared to a setting in which the regulator chooses the same policies $(\gamma, \delta)$ for both types of banks.

Corollary 3 The regulator cannot increase the expected surplus by offering different policies to good banks and bad banks.

\subsection{Additional negative externalities}

We study below the impact of negative externalities in case of banking failure at $t=2$ on the optimal policies set by the regulator at $t=0$. As in Bleck and Opp (2019), risktaking entails a social cost: if a bank with a risky loan fails at $t=2$, the regulator incurs a cost $\mathcal{K}>0$. This social cost can be interpreted as an increase in systemic risk or as the social cost from insufficient lending as a result of bank failures. ${ }^{18}$ As a result, at $t=1$, the regulator restructures a risky loan if and only if

$$
q \alpha+(1-q) \mathcal{L}>q \alpha+(1-q)(\mathbb{E}(p \mid s) \beta-(1-\mathbb{E}(p \mid s)) \mathcal{K})
$$

which is equivalent to

$$
\frac{\mathcal{L}+\mathcal{K}}{\beta+\mathcal{K}}>\mathbb{E}(p \mid s)=\delta s+(1-\delta) p_{m}
$$

\footnotetext{
${ }^{18}$ See Greenwood, Landier and Thesmar (2015) for a discussion of the negative externalities that exist in the banking industry.
} 
Given the negative externalities $\mathcal{K}$, the restructuring threshold is now defined as

$$
\tau(\delta) \equiv \begin{cases}1 & \text { if } 0 \leq \delta<\frac{\frac{\mathcal{L}+\mathcal{K}}{\beta+\mathcal{K}}-p_{m}}{1-p_{m}} \\ \frac{1}{\delta}\left(\frac{\mathcal{L}+\mathcal{K}}{\beta+\mathcal{K}}-(1-\delta) p_{m}\right) & \text { otherwise }\end{cases}
$$

The bank's risk choice is not affected by this additional externality and is therefore the same as in our baseline model. The regulator's maximization problem becomes

$$
\max _{\gamma, \delta} \Sigma(\gamma, \delta)=(q \alpha+(1-q)(F(\tau(\delta)) \mathcal{L}+k(\delta) \beta-(1-k(\delta)) \mathcal{K})-1) \gamma E
$$

subject to the bank's incentive constraint (3.7).

As in our main model, we show that the regulator optimally chooses an imperfect reporting system if the payoff of safe loans is sufficiently large or if the proportion of safe loans is sufficiently large.

Lemma 5 The reporting system is not fully precise, i.e., $\delta^{*}<1$, if either the payoff of a safe loan $\alpha$ is large enough or the proportion of safe loans $q$ is large enough.

Lemma 5 states that the main result of our paper is the same in the presence of additional negative externalities. We then analyze the impact of banking failure externalities on the optimal capital requirement and the optimal precision of the reporting system.

Corollary 4 If the proportion $q$ of safe loans is sufficiently large and $f^{\prime}\left(\tau\left(\delta^{*}\right)\right)$ is positive or sufficiently close to 0 , then the precision of the reporting system $\delta^{*}$ decreases in $\mathcal{K}$ whereas the capital requirement $\gamma^{*}$ increases with $\mathcal{K}$.

As the cost $\mathcal{K}$ of banking failures increases, restructuring becomes more attractive for the regulator. Hence, the regulator optimally designs a less precise reporting system

\footnotetext{
${ }^{19}$ The assumption equivalent to $\mathcal{L}>p_{m} \beta$ in the baseline model is $\mathcal{L}+\mathcal{K}>p_{m}(\beta+\mathcal{K})$ in this alternative setup.
} 
in order to increase the likelihood of restructuring. This increase in the likelihood of restructuring curbs the bank's risk-taking incentives, which implies that the regulator sets a looser capital requirement.

\subsection{Optimal persuasion}

In this extension, we study an alternative reporting system and we show that our main result, i.e., that the regulator may rely on imprecise information system still holds. In our baseline model, we assume that the regulator only controls the probability that the interim signal $s$ is noisy but we take the distribution of the noise as a given. Here, we assume that the regulator controls the entire distribution of the accounting signal (Kamenica and Gentzkow 2011) and therefore solve for the optimal persuasion mechanism.

Specifically, we now assume that the information system chosen at $t=0$ is a function $\delta(p)$ which prescribes the probability that the accounting signal $s=p$ is the true probability of default. We slightly generalize the baseline assumption about the noise distribution by assuming that the distribution of the noise is the c.d.f. of $p \mid \delta(p)=0$; this nests as a special case the baseline model since, if $\delta($.$) is a constant, the noise distribution is simply F($.$) .$ This avoids the case in which the noise mistakenly sends accounting reports for which the regulator would like to know the true probability of default $(\delta(p)=1)$. In the appendix 1 , we prove that doing so is optimal among all persuasion mechanisms in our game. ${ }^{20}$ The next lemma is critical to this analysis and reveals that the optimal accounting signal would feature asymmetric imprecision and characterizes the signal structure.

Lemma 6 The optimal information system is unique and such that there exists $\tau \geq \frac{\mathcal{L}}{\beta}$ with $\delta(p)=0$ if $p<\tau$, in which case the accounting signal $s$ is drawn from $p \mid p \leq \tau$, and

\footnotetext{
${ }^{20} \mathrm{As}$ is common in persuasion, the mechanism is not unique if we let the regulator use other functional forms $G($.$) (e.g., which could be other distributions with support on \left[0, \tau^{*}\right]$ ). However, a noise distribution $G($.$) that corresponds to the conditional distribution of p$ is natural for our setting since it assumes the noise is drawn from the distribution of the set of probabilities of default consistent with imprecision. This formulation also implies that there is a well-defined continuous distribution for the accounting signals and, hence, for the income construct in our model.
} 
$\delta(p)=1$ if $p>\tau$, in which case the accounting signal is $s=p$.

Similar to the baseline model, we make two assumptions to rule out cases in which the analysis is straightforward and no imprecision optimally, namely,

$$
\mathbb{E}(p \mid p \leq \tau) \beta \leq \mathcal{L} \leq \tau \beta
$$

and $^{21}$

$\left.\left.\max \left(\frac{\alpha-1}{\beta-1}, \frac{1-q \alpha}{(1-q) \beta}-F(\mathcal{L} / \beta) \mathcal{L} / \beta\right)\right)<\int_{\mathcal{L} / \beta}^{1} p f(p) d p<\min \left(\frac{\alpha}{\beta}, 1 / \beta-F(\mathcal{L} / \beta) \mathcal{L} / \beta\right)\right)$.

At $t=1$, given that $p>\tau$, the signal sent by the reporting system is $s=p$ and the equity of the bank is

$$
E_{1}=A(q \alpha+(1-q) p \beta)-D>A(q \alpha+(1-q) \mathcal{L})-D
$$

so that the bank may violate the capital requirement $\gamma$ but the regulator never restructures. Otherwise, if $p \leq \tau$, the signal sent by the reporting system is pure noise and the equity of the bank is

$$
E_{1}=A(q \alpha+(1-q) \mathbb{E}(p \mid p \leq \tau) \beta)-D<A(q \alpha+(1-q) \mathcal{L})-D<E
$$

so that the bank violates the capital requirement $\gamma$ and the regulator restructures a risky loan. The regulator's maximization problem is therefore equivalent to choosing a restruc-

\footnotetext{
${ }^{21}$ This assumption rules out degenerate cases for which the analysis becomes straightforward. The first part of the left-hand side of the inequality states that the bank originates a high-risk loan portfolio if the bank size is arbitrarily large and the reporting system is fully informative. The second part requires the low-risk loan portfolio to have positive expected value because, otherwise, the regulator would always induce $\gamma=1$. The first part of the right-hand side of the inequality rules out parameter values for which the risk-shifting problem is so severe that the bank would only lend its own equity, $E$. The second part of the right-hand side of the inequality guarantees that the high-risk loan portfolio is value-destroying.
} 
turing threshold $\tau$ and a capital requirement $\gamma$ to maximize the expected surplus:

$$
\max _{\gamma, \tau \in[0,1]}\left(q \alpha+(1-q)\left(\int_{0}^{\tau} f(p) d p \mathcal{L}+\int_{\tau}^{1} p f(p) d p \beta\right)-1\right) \gamma E
$$

s.t.

$$
\begin{aligned}
\int_{0}^{\tau} f(p) d p \frac{\mathcal{L}}{\beta}-\int_{0}^{\tau} p f(p) d p & \geq 0 \\
\tau-\frac{\mathcal{L}}{\beta} & \geq 0 \\
\gamma \alpha-(\gamma-1)-\int_{\tau}^{1} p f(p) d p(\gamma \beta-(\gamma-1)) & \geq 0,
\end{aligned}
$$

where (4.12) and (4.13) are the left-hand and right-hand side of (4.9), respectively, and (4.14) is the bank's incentive constraint.

Proposition 5 The optimal restructuring threshold $\tau^{*}$ is strictly higher than the restructuring threshold when the reporting system is fully informative: $\tau^{*}>\mathcal{L} / \beta$.

Recall that the restructuring decision is also governed by an ex-post constraint which bars policies in which the regulator would restructure a risky loan with greater value if it were continued. The higher the restructuring threshold, the more this constraint becomes difficult to satisfy. Specifically, the ex-post constraint is satisfied if and only if $\tau \leq \bar{\tau}$, where $\bar{\tau}$

$$
\int_{0}^{\bar{\tau}} p f(p) d p=F(\bar{\tau}) \frac{\mathcal{L}}{\beta}
$$

Note that $\bar{\tau}<1$ if and only if $\mathbb{E}(p) \beta>\mathcal{L}$. To characterize the optimal threshold, note that $\Sigma^{\prime}(\bar{\tau})<0$ is a sufficient condition for the existence of an interior solution $\tau^{*}<\bar{\tau}$ since, then, the regulator would increase expected surplus by reducing the restructuring threshold. 
Proposition 6 The restructuring threshold is set at the maximum level $\tau^{*}=\bar{\tau}$ if and only if the proportion of safe loans is high enough, i.e.,

$$
q>\bar{q} \equiv \frac{\nu}{(\alpha-1)(\beta-\alpha) \bar{\tau}+\nu} \in(0,1)
$$

where $\nu$ is not a function of $q$ and is defined in the appendix.

The intuition for proposition 6 is given in two steps, starting with a comparative static in $q$ in this paragraph and followed by the rationale for $\tau^{*}=\bar{\tau}$. When $q$ is large, there is a greater net benefit from increasing leverage. With a single regulation, this leverage is fully determined by the incentive constraint, so that there is little the regulator can do to increase it further. Here, on the other hand, the regulator can increase excessive restructurings in risky loans. Putting both regulatory channels together, we conclude that the regulator (weakly) increases the restructuring threshold $\tau^{*}$ in response to an increase in the proportion of safe loans $q$. This requires to increase imprecision by pooling more positive realizations of $p$ into the restructuring region.

Continuing on this logic, expectations about failing banks increase as a function of $q$, and it becomes increasingly difficult for the regulator to credibly restructure. When $q$ becomes greater than $\bar{q}$, the required restructuring threshold to implement the ideal leverage would be above $\bar{\tau}$. At this point, the ex-post restructuring constraint $\mathbb{E}(p \mid p \leq$ $\left.\tau^{*}\right) \leq \mathcal{L} / \beta$ becomes binding and the regulator implements the maximal credible threshold $\bar{\tau}$. Put differently, when the proportion of safe loans is sufficiently large, the regulator implements the maximal credible level of restructuring. Naturally, when this point $q \geq \bar{q}$ is reached, the credible level of restructuring no longer depends on $q$ since the regulator does not restructure a safe loan. We summarize this comparative static, as well as the other comparative statics (which follow from an analogous intuitions as in the baseline model) in the next corollary. 
Corollary 5 The optimal capital requirement $\gamma^{*}$ and the restructuring threshold $\tau^{*}$ are increasing in the proportion of safe loans $q$, the payoff of a safe loan $\alpha$ and the restructuring value $\mathcal{L}$, strictly if $q \leq \bar{q}$. Further, if $q \geq \bar{q}$, the optimal capital requirement $\gamma^{*}$ and the restructuring threshold $\tau^{*}$ are decreasing in the payoff of a risky loan $\beta$. Otherwise, if $q<\bar{q}$, for any parameter values such that $\partial \tau^{*} / \partial \beta<0$ or $\partial \gamma^{*} / \partial \beta>0, \tau^{*}$ and $\gamma^{*}$ vary in the same direction as a function of $\beta$.

The effect of $\beta$ depends on whether the ex-post restructuring constraint $\bar{\tau}$ is reached. If $\tau^{*}=\bar{\tau}$, the constraining factor is to make the restructuring credible, so that higher $\beta$, because it makes it more tempting for the regulator to continue, leads to a reduction in the threshold and a lower leverage. Credit markets with a larger proportion of risky loans, $q<\bar{q}$, are the only case where the optimal capital requirement and the restructuring may enter in different directions, as a result of the two trade-offs discussed earlier.

Finally, table 2 summarizes the comparative statics of the model with an alternative reporting system, with the main observation being that, for all variables except for the payoff $\beta$ of a risky loan, the reporting system and the capital requirement move in tandem and are very similar to those in the baseline model. In summary, the main differences when the regulator is able to use an optimal persuasion mechanism is that, when the proportion of safe loans becomes large enough, it may no longer be desirable to increase restructuring further in response to a change in the environment.

\section{An application to expected loan loss provisioning}

Our model informs the debate regarding the optimal provisioning model for loan losses that has received much attention since the 2007-08 financial crisis. Under the previous IASB (IAS 39) and FASB (FAS 114) standards, the accounting model for recognizing loan losses was referred to as an incurred loss model. Such a model required the recording of loan losses that have been incurred as of the balance sheet date, rather than of probable 


\begin{tabular}{l|l||cccc} 
& & $\alpha$ & $\mathcal{L}$ & $q$ & $\beta$ \\
\hline Joint maximization & $\gamma^{*}$ & + & + & 0 & - \\
with $q>\bar{q}$ & $\tau^{*}$ & 0 & + & 0 & - \\
Joint maximization & $\gamma^{*}$ & + & + & + & $+/-$ \\
with $q \leq \bar{q}$ & $\tau^{*}$ & + & + & + & $+/-$ \\
\hline Exogenous precision & $\gamma^{*}$ & + & 0 & 0 & - \\
with $\tau \leq \bar{\tau}$ & & & & & + \\
\hline Exogenous capital re- & $\tau^{*}$ & - & 0 & 0 & + \\
quirement with $\gamma_{f}>$ & & & & & \\
$\quad 1-\int_{\frac{\mathcal{L}}{\beta}}^{1} p f(p) d p$ & & & & &
\end{tabular}

Table 2: Comparative statics with optimal persuasion

future losses. The new expected loan loss standards (CECL and IFRS 9) have a more forward-looking approach that emphasizes shifts to the probability of future loan losses, even if no triggering events have yet occurred. In particular, relative to an incurred loss model, an expected loss model requires banks to use early signals about future loan losses and being inherently imprecise, such early signals could, in turn, induce more frequent and potentially excessive restructurings. We view our mechanism design problem as an efficient expected loss, in the sense that banks report information about a probability of default $p$ before the realization of loan payoffs.

We model the incurred loss model as follows. At $t=1$, with probability $\chi \in[0,1]$, the accounting system perfectly reveals the probability of default $p$. Otherwise, with probability $1-\chi$, the accounting system does not provide any signal. In that latter case, if the accounting system does not provide any information at $t=1$, then the bank's equity is $E_{1}=E$, which implies that the bank does not violate the capital requirement and the regulator cannot restructure. Under the incurred loss model, the regulator's objective function becomes

$$
\max _{\gamma, \delta}\left(q \alpha+(1-q)\left(\left(\chi F\left(\frac{\mathcal{L}}{\beta}\right)+(1-\chi) F(\tau(\delta))\right) \mathcal{L}+(\chi k(1)+(1-\chi) k(\delta)) \beta\right)-1\right) \gamma E
$$


s.t.

$$
\gamma=\frac{1-\chi k(1)-(1-\chi) k(\delta)}{(\chi k(1)+(1-\chi) k(\delta))(\beta-1)-(\alpha-1)}
$$

Compared to our baseline model, the presence of this other signal sets an upper bound on the restructuring threshold. In particular, in the extreme case in which the regulator always learns $p$ perfectly, i.e., $\chi=1$, there cannot be any excessive regulatory restructuring.

Corollary 6 If $\int_{\frac{\mathcal{L}}{\beta}}^{1}\left(p-p_{m}\right) f(p) d p$ is sufficiently small and $(2 \chi k(1)-1)(\beta-1)>\alpha-1$, then the optimal precision $\delta^{*}<1$ decreases with the probability $\chi$.

The ex-ante precision of the reporting system, $\chi+(1-\chi) \delta$, increases in $\chi$ for a given $\delta$. As $\chi$ increases, the regulator reduces the precision $\delta^{*}$ in order to offset the impact on the ex-ante precision. As a result, the effect of an increase in $\chi$ on the ex-ante precision and on the capital requirement is ambiguous.

Our model generates several interesting insights about the desirability of an expected loss model. First, the efficient expected loan loss provisioning model should not provide perfect information about the probability of default because it would imply excessively low bank leverage (that is, prudential regulations that are too strict). But, not surprisingly, by relying on less precise information about the probability of default, such a reporting system causes excessive restructurings, especially in environments where the proportion of risky loans is high enough so that such restructurings could be socially costly.

Second, the comparative statics of our model on the restructuring threshold shed light on the extent to which the reporting system should rely on imprecise information about loan losses. Higher permissible leverages go hand in hand with reporting systems that are tilted towards an expected loss model that rely on less precise information. In particular, if the payoff of safe loans or the restructuring value of risky loans are higher, or the proportion of safe loans is higher, the regulator tends to readjust toward an expected loss model with less precise information, increasing bank leverage in the process. We view these settings as environments when the economy as a whole features more favorable conditions so that an 
expected loss model with less precise information is particularly suitable for expansionary credit periods. This result also provides a rationale for the dynamic loan loss provisioning that has been implemented in Spain in the early 2000s (Jiménez, Ongena, Peydró and Saurina 2017).

\section{Concluding remarks}

We develop a simple integrated role of accounting standards and restrictions on bank lending, under the assumption that accounting standards can influence some of the information available to regulators. ${ }^{22}$ This is, of course, a stylized view in that prudential regulators may have access to other sources of information. However, we believe it also reflects public pressures to intervene based on information that is publicly known. Standard setters may struggle to persuade the public that a bank that can provide value on its own should be restructured, especially given that such regulatory interventions come with significant social consequences. This creates a time-inconsistency problem which can be affected by the information released about a bank in its financial statements.

An implication of our analysis is that the current focus on providing decision-useful information to investors can be detrimental to banks. Put differently, in a strategic setting, more decision-useful information can lead to worse interventions and demand stricter prudential regulations. Standard setters have noted that they are not equipped to exert judgment about prudential issues, since these involve policy judgments: our analysis suggests benefits in creating a stronger articulation between the missions and encouraging communication between the two bodies.

Our results also provide a rationale for the new expected loss provisioning model re-

\footnotetext{
${ }^{22} \mathrm{An}$ implication of integration, which we do not examine in this study, is a uniformization of standards across different jurisdictions subject to joint prudential regulations (e.g., Basel agreements). This creates trade-offs as to desirable levels of harmonization in accounting standards (Stecher and Suijs 2012, Friedman and Heinle 2016) that we do not consider in this study but will likely become important parts of the question with more clearly stated joint objectives.
} 
cently adopted by standard setters. More work is needed to analyze further the optimal loan loss provisioning model and to fully understand the tradeoffs at stake. In particular, the procyclical impact of the provisions for loan losses on bank lending has been heavily debated since the 2007-08 financial crisis. Our static model does not speak to this question and we would need a dynamic setting to highlight the features of the optimal provisioning model. We leave this important question for future research. 


\section{Bibliography}

Acharya, Viral V, and Tanju Yorulmazer (2007) 'Too many to fail-an analysis of timeinconsistency in bank closure policies.' Journal of Financial Intermediation 16(1), 131

Allen, Franklin, and Elena Carletti (2008) 'Mark-to-market accounting and liquidity pricing.' Journal of Accounting and Economics 45(2), 358-378

Arya, Anil, Jonathan C Glover, and Konduru Sivaramakrishnan (1997) 'The interaction between decision and control problems and the value of information.' Accounting Review pp. 561-574

Barth, Mary E, and Wayne R Landsman (2010) 'How did financial reporting contribute to the financial crisis?' European Accounting Review 19(3), 399-423

Beatty, Anne, and Scott Liao (2011) 'Do delays in expected loss recognition affect banks' willingness to lend?' Journal of Accounting and Economics 52(1), 1-20

Bleck, Alexander (2018) 'Regulating bank leverage.' Journal of Financial Economic Policy

Bleck, Alexander, and Christian C Opp (2019) 'Optimal risk-sensitivity of bank regulation'

Bleck, Alexander, and Pingyang Gao (2017) 'Mark-to-market, loan retention, and loan origination'

Bleck, Alexander, and Xuewen Liu (2007) 'Market transparency and the accounting regime.' Journal of Accounting Research 45(2), 229-256

Boot, Arnoud WA, and Anjan V Thakor (1993) 'Self-interested bank regulation.' American Economic Review pp. 206-212

Boyd, John H, and Gianni De Nicolo (2005) 'The theory of bank risk taking and competition revisited.' Journal of Finance 60(3), 1329-1343 
Burkhardt, Katrin, and Roland Strausz (2009) 'Accounting transparency and the asset substitution problem.' Accounting Review 84(3), 689-712

Bushman, Robert M (2014) 'Thoughts on financial accounting and the banking industry.' Journal of Accounting and Economics 58(2-3), 384-395

Bushman, Robert M, and Christopher D Williams (2012) 'Accounting discretion, loan loss provisioning, and discipline of banks' risk-taking.' Journal of Accounting and Economics 54(1), 1-18

Campbell, Tim S, Yuk-Shee Chan, and Anthony M Marino (1992) 'An incentive-based theory of bank regulation.' Journal of Financial Intermediation 2(3), 255-276

Chan, Yuk-Shee, Stuart I Greenbaum, and Anjan V Thakor (1992) 'Is fairly priced deposit insurance possible?' Journal of Finance 47(1), 227-245

Chircop, Justin, and Zoltán Novotny-Farkas (2016) 'The economic consequences of extending the use of fair value accounting in regulatory capital calculations.' Journal of Accounting and Economics 62(2), 183-203

Corona, Carlos, Lin Nan, and Gaoqing Zhang (2015) 'Accounting information quality, interbank competition, and bank risk-taking.' Accounting Review 90(3), 967-985

_ (2019a) 'Banks' asset reporting frequency and capital regulation: An analysis of discretionary use of fair-value accounting.' Accounting Review 94(2), 157-178 (2019b) 'The coordination role of stress tests in bank risk-taking.' Journal of Accounting Research 57(5), 1161-1200

Crémer, Jacques (1995) 'Arm's length relationships.' Quarterly Journal of Economics $110(2), 275-295$ 
Dewatripont, Mathias, and Jean Tirole (1994) The prudential regulation of banks (MIT Press)

Ellul, Andrew, Chotibhak Jotikasthira, Christian T Lundblad, and Yihui Wang (2015) 'Is historical cost accounting a panacea? market stress, incentive distortions, and gains trading.' Journal of Finance 70(6), 2489-2538

Freixas, Xavier, and Jean-Charles Rochet (2008) Microeconomics of banking (MIT Press)

Friedman, Henry L, and Mirko S Heinle (2016) 'Taste, information, and asset prices: Implications for the valuation of csr.' Review of Accounting Studies 21(3), 740-767

Ganuza, Juan-José, and Jose Penalva (2019) 'Information disclosure in optimal auctions.' International Journal of Industrial Organization 63, 460-479

Gao, Pingyang, and Xu Jiang (2018) 'Reporting choices in the shadow of bank runs.' Journal of Accounting and Economics 65(1), 85-108

Giammarino, Ronald M, Tracy R Lewis, and David EM Sappington (1993) 'An incentive approach to banking regulation.' Journal of Finance 48(4), 1523-1542

Goex, Robert F., and Alfred Wagenhofer (2009) 'Optimal impairment rules.' Journal of Accounting and Economics 48(1), 2-16

Goldstein, Itay, and Haresh Sapra (2014) 'Should banks' stress test results be disclosed? an analysis of the costs and benefits.' Foundations and Trends in Finance 8(1), 1-54

Gorton, Gary, and Andrew Winton (2017) 'Liquidity provision, bank capital, and the macroeconomy.' Journal of Money, Credit and Banking 49(1), 5-37

Granja, Joao, Gregor Matvos, and Amit Seru (2017) 'Selling failed banks.' Journal of Finance 72(4), 1723-1784 
Greenwood, Robin, Augustin Landier, and David Thesmar (2015) 'Vulnerable banks.' Journal of Financial Economics 115(3), 471-485

Guttman, Ilan, and Iván Marinovic (2018) 'Debt contracts in the presence of performance manipulation.' Review of Accounting Studies 23(3), 1005-1041

Heaton, John C, Deborah Lucas, and Robert L McDonald (2010) 'Is mark-to-market accounting destabilizing? analysis and implications for policy.' Journal of Monetary Economics 57(1), 64-75

Hellmann, Thomas F, Kevin C Murdock, and Joseph E Stiglitz (2000) 'Liberalization, moral hazard in banking, and prudential regulation: Are capital requirements enough?' American Economic Review pp. 147-165

Holmstrom, Bengt, and Jean Tirole (1997) 'Financial intermediation, loanable funds, and the real sector.' Quarterly Journal of Economics 112(3), 663-691

Jiménez, Gabriel, Steven Ongena, José-Luis Peydró, and Jesús Saurina (2017) 'Macroprudential policy, countercyclical bank capital buffers, and credit supply: evidence from the spanish dynamic provisioning experiments.' Journal of Political Economy $125(6), 2126-2177$

Kamenica, Emir, and Matthew Gentzkow (2011) 'Bayesian persuasion.' American Economic Review 101(6), 2590-2615

Kanodia, Chandra, and Haresh Sapra (2016) 'A real effects perspective to accounting measurement and disclosure: Implications and insights for future research.' Journal of Accounting Research 54(2), 623-676

Kydland, Finn E, and Edward C Prescott (1977) 'Rules rather than discretion: The inconsistency of optimal plans.' Journal of Political Economy 85(3), 473-491 
Li, Jing (2017) 'Accounting for banks, capital regulation and risk-taking.' Journal of Banking and Finance 74, 102-121

Liang, Pierre Jinghong, and Gaoqing Zhang (2019) 'On the social value of accounting objectivity in financial stability.' Accounting Review 94(1), 229-248

Liang, Pierre Jinghong, Madhav V Rajan, and Korok Ray (2008) 'Optimal team size and monitoring in organizations.' Accounting Review 83(3), 789-822

Lu, Tong, Haresh Sapra, and Ajay Subramanian (2019) 'Agency conflicts, bank capital regulation, and marking-to-market.' Accounting Review 94(6), 365-384

Mahieux, Lucas (2019) 'Fair value accounting, transparency and financial stability'

Mahieux, Lucas, Haresh Sapra, and Gaoqing Zhang (2020) 'Cecl: Timely loan loss provisioning and banking regulation'

Martinez-Miera, David, and Rafael Repullo (2010) 'Does competition reduce the risk of bank failure?' Review of Financial Studies 23(10), 3638-3664

Plantin, Guillaume, Haresh Sapra, and Hyun Song Shin (2008) 'Marking to market: Panacea or pandora's box?' Journal of Accounting Research 46(2), 435-460

Repullo, Rafael (2004) 'Capital requirements, market power, and risk-taking in banking.' Journal of Financial Intermediation 13(2), 156-182

Rochet, Jean-Charles (2004) 'Macroeconomic shocks and banking supervision.' Journal of Financial Stability 1(1), 93-110

Stecher, Jack, and Jeroen Suijs (2012) 'Hail, procrustes! harmonized accounting standards as a procrustean bed.' Journal of Accounting and Public Policy 31(4), 341-355

Stern, Gary H, and Ron J Feldman (2004) Too big to fail: The hazards of bank bailouts (Brookings Institution Press) 
Zhang, Gaoqing (2020) 'Competition and opacity in the financial system.' Management Science 


\section{Appendix}

\section{Appendix 1: proofs}

\section{Proof of lemmas 1-4:}

At $t=1$, the regulator optimally restructures a risky loan if and only if the bank violates the capital requirement $\gamma$ and $q \alpha+(1-q) \mathbb{E}(p \mid s) \beta<q \alpha+(1-q) \mathcal{L}$. First, note that the condition $q \alpha+(1-q) \mathbb{E}(p \mid s) \beta<$ $q \alpha+(1-q) \mathcal{L}$ is equivalent to $p<\tau(\delta)$, where $\tau(\delta)$ is defined as follows

$$
\tau(\delta) \equiv \begin{cases}1 & \text { if } 0 \leq \delta<\frac{\frac{\mathcal{L}}{\beta}-p_{m}}{1-p_{m}} \\ \frac{1}{\delta}\left(\frac{\mathcal{L}}{\beta}-(1-\delta) p_{m}\right) & \text { otherwise }\end{cases}
$$

Further, assumption 2 implies that $q \alpha+(1-q) \mathcal{L}<1$. As a result, if the bank chooses the maximal bank size at $t=0$, i.e., $A=\gamma E$, the bank violates the capital requirement $\gamma$ if and only if $q \alpha+(1-q) \mathbb{E}(p \mid s) \beta<1$. Otherwise, if the bank chooses $A<\gamma E$, then the bank violates the capital requirement if and only if

$$
\frac{A(q \alpha+(1-q) \mathbb{E}(p \mid s) \beta)-D}{A(q \alpha+(1-q) \mathbb{E}(p \mid s) \beta)}<1 / \gamma,
$$

which is equivalent to

$$
q \alpha+(1-q) \mathbb{E}(p \mid s) \beta<\frac{\gamma(A-E)}{(\gamma-1) A} .
$$

Hence, if the bank chooses $A$ such that

$$
\frac{\gamma(A-E)}{(\gamma-1) A} \geq q \alpha+(1-q) \mathcal{L},
$$

the regulator restructures a risky loan if and only if $p<\tau(\delta)$. Condition (6.2) is equivalent to

$$
A \geq \frac{\gamma E}{\gamma-(\gamma-1)(q \alpha+(1-q) \mathcal{L})} .
$$

As we show at the end of this proof, the bank's incentive constraint then binds in equilibrium. As a result, the equilibrium leverage is

$$
A=E \frac{1-k(\delta)}{k(\delta)(\beta-1)-(\alpha-1)} .
$$

Given this leverage, assumption 4 implies that condition (6.2) is always satisfied in equilibrium.

Next, we show that conditional on originating a loan portfolio, the bank's expected payoff is increasing 
in $A$ for any $\delta$. The bank with a risky loan receives a zero payoff with restructuring but, otherwise, receives a payoff $A \alpha-(A-E)$ when continuing with a safe loan and $\mathbb{E}(p \mid s)(A \beta-(A-E))$ when continuing with a risky loan. We have

$$
\begin{aligned}
\frac{\partial A \alpha-(A-E)}{\partial A} & =\alpha-1>0, \\
\frac{\partial \mathbb{E}(p \mid s)(A \beta-(A-E))}{\partial A} & =\mathbb{E}(p \mid s)(\beta-1)>0 .
\end{aligned}
$$

As a result, for a given capital requirement $\gamma$, the bank chooses $A^{*}$ such that $A^{*}=\gamma E$. Further, the regulator restructures a risky loan if and only $p<\tau(\delta)$.

Lastly, we prove that the risk-shifting incentive constraint of the bank (3.5) binds in equilibrium. For a capital requirement $\gamma$, if the incentive constraint is not satisfied, then the bank originates a high-risk loan portfolio with negative NPV. However, the regulator is then better-off setting $\gamma^{*}=1$. The incentive constraint becomes $\alpha>k(\delta) \beta$. Assumption 1 implies that $\alpha>k(1) \beta \geq k(\delta) \beta$. As a result, the incentive constraint is satisfied in equilibrium. Further, if the incentive constraint does not bind and is strictly satisfied in equilibrium, then the bank always originates a low-risk loan portfolio. However, the regulator is then better-off setting $\gamma=\infty$. The incentive constraint becomes $\alpha-1>k(\delta)(\beta-1)$. Assumption 2 implies that this is not satisfied if $\delta>\delta_{0}$. As a result, the risk-shifting incentive constraint of the bank (3.5) binds in equilibrium.

Proof of proposition 1: We solve program $(P)$ with commitment, that is, replacing $\tau(\delta)$ by $\tau_{c}$ and optimizing in $\left(\gamma, \tau_{c}, \delta\right)$. Substituting $\gamma$ from the binding incentive constraint into the objective function, the regulator's maximization problem becomes

$$
\begin{aligned}
\max _{\tau_{c}, \delta} \Sigma_{b}(\delta) \equiv\left(q \alpha+(1-q)\left(F\left(\tau_{c}\right) \mathcal{L}+\int_{\tau_{c}}^{1}(\delta p\right.\right. & \left.\left.\left.+(1-\delta) p_{m}\right) f(p) d p \beta\right)-1\right) \\
& \times \frac{1-\int_{\tau_{c}}^{1}\left(\delta p+(1-\delta) p_{m}\right) f(p) d p}{\int_{\tau_{c}}^{1}\left(\delta p+(1-\delta) p_{m}\right) f(p) d p(\beta-1)-(\alpha-1)} E
\end{aligned}
$$

Taking the first-order condition with respect to $\delta$ yields

$$
\begin{aligned}
\frac{\partial \Sigma_{b}}{\partial \delta}= & \frac{(1-q) \beta\left(1-\int_{\tau_{c}}^{1}\left(\delta p+(1-\delta) p_{m}\right) f(p) d p\right)}{\left(\int_{\tau_{c}}^{1}\left(\delta p+(1-\delta) p_{m}\right) f(p) d p(\beta-1)-(\alpha-1)\right)} \\
& +\frac{\left(q \alpha+(1-q)\left(F\left(\tau_{c}\right) \mathcal{L}+\int_{\tau_{c}}^{1}\left(\delta p+(1-\delta) p_{m}\right) f(p) d p \beta\right)-1\right)(\beta-\alpha)}{\left(\int_{\tau_{c}}^{1}\left(\delta p+(1-\delta) p_{m}\right) f(p) d p(\beta-1)-(\alpha-1)\right)^{2}}>0 .
\end{aligned}
$$


As a result, $\delta^{*}=1$. Next, taking the first-order condition with respect to $\tau_{c}$ yields

$$
\begin{aligned}
\frac{\partial \Sigma_{b}}{\partial \tau_{c}}=\left((1-q)\left(\mathcal{L}-\left(\delta \tau_{c}+(1-\delta) p_{m}\right) \beta\right)\right) & \underbrace{\left(1-\int_{\tau_{c}}^{1}\left(\delta p+(1-\delta) p_{m}\right) f(p) d p\right)}_{>0} \\
\times & \underbrace{\left(\int_{\tau_{c}}^{1}\left(\delta p+(1-\delta) p_{m}\right) f(p) d p(\beta-1)-(\alpha-1)\right)}_{>0} \\
+ & \underbrace{\left(q \alpha+(1-q)\left(F\left(\tau_{c}\right) \mathcal{L}+\int_{\tau_{c}}^{1}\left(\delta p+(1-\delta) p_{m}\right) f(p) d p \beta\right)-1\right)(\beta-\alpha)\left(\delta \tau_{c}+(1-\delta) p_{m}\right)}_{>0} .
\end{aligned}
$$

First, assumption 2 implies that always restructuring a risky loan, i.e., $\tau_{c}=1$, is suboptimal for the regulator. Second, if the regulator never restructures, i.e, the regulator sets $\tau_{c}=0$, then the surplus is

$$
q \alpha+(1-q) \int_{0}^{1} p f(p) d p \beta-1<q \alpha+(1-q) \mathcal{L}-1
$$

which is negative by assumption 2 . Hence, it must be the case that the solution $\tau_{c}^{*} \in(0,1)$ satisfies $\frac{\partial \Sigma_{b}}{\partial \tau_{c}}=0$. Hence, it should be the case that the left-hand side is negative, which implies that

$$
(1-q)\left(\mathcal{L}-\left(\delta \tau_{c}+(1-\delta) p_{m}\right) \beta\right)<0
$$

As a result, given that $\delta^{*}=1$, we have $\tau_{c}^{*}>\frac{\mathcal{L}}{\beta}$.

Proof of proposition 2 and corollary 1: Suppose the precision of the reporting system $\delta=\delta_{f}$ is fixed and $\delta_{f} \geq \delta_{0}$ (from the definition of $\delta_{0}$, if $\delta<\delta_{0}$, then a low-risk loan portfolio has ex-ante negative value). It follows from lemma 4 that the optimal capital requirement is $\gamma^{*}=\frac{1-k\left(\delta_{f}\right)}{k\left(\delta_{f}\right)(\beta-1)-(\alpha-1)}$. Recalling that $k\left(\delta_{f}\right)=\int_{\tau\left(\delta_{f}\right)}^{1}\left(\delta_{f} p+\left(1-\delta_{f}\right) p_{m}\right) f(p) d p$, is immediate from (6.1) that $k\left(\delta_{f}\right)$ is increasing in $\beta$ and $\delta_{f}$, and decreasing in $\mathcal{L}$. It then follows that

$$
\begin{aligned}
\frac{\partial \gamma^{*}}{\partial \alpha} & =\frac{1-k\left(\delta_{f}\right)}{\left(k\left(\delta_{f}\right)(\beta-1)-(\alpha-1)\right)^{2}}>0, \\
\frac{\partial \gamma^{*}}{\partial \mathcal{L}} & =\frac{-\frac{\partial k\left(\delta_{f}\right)}{\partial \mathcal{L}}\left(k\left(\delta_{f}\right)(\beta-1)-(\alpha-1)\right)-\left(1-k\left(\delta_{f}\right)\right) \frac{\partial k\left(\delta_{f}\right)}{\partial \mathcal{L}}(\beta-1)}{\left(k\left(\delta_{f}\right)(\beta-1)-(\alpha-1)\right)^{2}}>0 \\
\frac{\partial \gamma^{*}}{\partial \beta} & =\frac{-\frac{\partial k\left(\delta_{f}\right)}{\partial \beta}\left(k\left(\delta_{f}\right)(\beta-1)-(\alpha-1)\right)-\left(1-k\left(\delta_{f}\right)\right)\left(\frac{\partial k\left(\delta_{f}\right)}{\partial \beta}(\beta-1)+k\left(\delta_{f}\right)\right)}{\left(k\left(\delta_{f}\right)(\beta-1)-(\alpha-1)\right)^{2}}<0 \\
\frac{\partial \gamma^{*}}{\partial \delta_{f}} & =\frac{-\frac{\partial k\left(\delta_{f}\right)}{\partial \delta_{f}}\left(k\left(\delta_{f}\right)(\beta-1)-(\alpha-1)\right)-\left(1-k\left(\delta_{f}\right)\right) \frac{\partial k\left(\delta_{f}\right)}{\partial \delta_{f}}(\beta-1)}{\left(k\left(\delta_{f}\right)(\beta-1)-(\alpha-1)\right)^{2}}<0 .
\end{aligned}
$$


Suppose next that $\gamma=\gamma_{f}$ is fixed. First, suppose that $\gamma_{f}<\frac{1-k(1)}{k(1)(\beta-1)-(\alpha-1)}$, this implies that the bank's incentive constraint is satisfied for any $\delta$. As a result, the regulator maximizes the low-risk loan portfolio surplus and sets $\delta^{*}=1$. Next, suppose that $\gamma_{f} \geq \frac{1-k(1)}{k(1)(\beta-1)-(\alpha-1)}$. We know from Lemma 4 that the optimal precision $\delta^{*}$ satisfies $k\left(\delta^{*}\right)=\frac{1+(\alpha-1) \gamma_{f}}{1+(\beta-1) \gamma_{f}} \in\left(\frac{\alpha}{\beta}, \frac{\alpha-1}{\beta-1}\right)$. Note that, if $0 \leq \delta<\frac{\frac{\mathcal{L}}{\beta}-p_{m}}{1-p_{m}}$, the function $k(\delta)$ is strictly increasing in $\delta$ with $k\left(\frac{\frac{\mathcal{L}}{\beta}-p_{m}}{1-p_{m}}\right)=0$ and $k(1)=\int_{\frac{\mathcal{L}}{\beta}}^{1} p f(p) d p>\frac{\alpha-1}{\beta-1}$ by assumption 2 . As a result, the function $k($.$) is invertible, i.e., the solution is unique. We next derive the partial derivatives of$ the left-hand side and the right-hand side of equation $k\left(\delta^{*}\right)=\frac{1+(\alpha-1) \gamma_{f}}{1+(\beta-1) \gamma_{f}}$ :

$$
\begin{aligned}
& -\left(\delta^{*} \tau\left(\delta^{*}\right)+\left(1-\delta^{*}\right) p_{m}\right) f\left(\tau\left(\delta^{*}\right)\right) \frac{\partial \tau\left(\delta^{*}\right)}{\partial \alpha}+\int_{\tau\left(\delta^{*}\right)}^{1}\left(p-p_{m}\right) \frac{\partial \delta^{*}}{\partial \alpha} f(p) d p=\frac{\gamma_{f}}{1+(\beta-1) \gamma_{f}}>0 \\
& -\left(\delta^{*} \tau\left(\delta^{*}\right)+\left(1-\delta^{*}\right) p_{m}\right) f\left(\tau\left(\delta^{*}\right)\right) \frac{\partial \tau\left(\delta^{*}\right)}{\partial \mathcal{L}}+\int_{\tau\left(\delta^{*}\right)}^{1}\left(p-p_{m}\right) \frac{\partial \delta^{*}}{\partial \mathcal{L}} f(p) d p=0 \\
& -\left(\delta^{*} \tau\left(\delta^{*}\right)+\left(1-\delta^{*}\right) p_{m}\right) f\left(\tau\left(\delta^{*}\right)\right) \frac{\partial \tau\left(\delta^{*}\right)}{\partial \beta}+\int_{\tau\left(\delta^{*}\right)}^{1}\left(p-p_{m}\right) \frac{\partial \delta^{*}}{\partial \beta} f(p) d p=\frac{-\left(1+(\alpha-1) \gamma_{f}\right) \gamma_{f}}{\left(1+(\beta-1) \gamma_{f}\right)^{2}}<0 \\
& -\left(\delta^{*} \tau\left(\delta^{*}\right)+\left(1-\delta^{*}\right) p_{m}\right) f\left(\tau\left(\delta^{*}\right)\right) \frac{\partial \tau\left(\delta^{*}\right)}{\partial \gamma_{f}}+\int_{\tau\left(\delta^{*}\right)}^{1}\left(p-p_{m}\right) \frac{\partial \delta^{*}}{\partial \gamma_{f}} f(p) d p=\frac{\alpha-\beta}{\left(1+(\beta-1) \gamma_{f}\right)^{2}}<0 .
\end{aligned}
$$

Further, we know that

$$
\begin{aligned}
\frac{\partial \tau\left(\delta^{*}\right)}{\partial \mathcal{L}} & =\frac{1}{\delta^{*} \beta}-\frac{1}{\left(\delta^{*}\right)^{2}}\left(\frac{\mathcal{L}}{\beta}-p_{m}\right) \frac{\partial \delta^{*}}{\partial \mathcal{L}}, \\
\frac{\partial \tau\left(\delta^{*}\right)}{\partial \beta} & =-\frac{\mathcal{L}}{\delta^{*} \beta^{2}}-\frac{1}{\left(\delta^{*}\right)^{2}}\left(\frac{\mathcal{L}}{\beta}-p_{m}\right) \frac{\partial \delta^{*}}{\partial \beta}, \\
\frac{\partial \tau\left(\delta^{*}\right)}{\partial \delta^{*}} & =-\frac{1}{\left(\delta^{*}\right)^{2}}\left(\frac{\mathcal{L}}{\beta}-p_{m}\right)<0 .
\end{aligned}
$$

As a result, $\frac{\partial \delta^{*}}{\partial \alpha}>0, \frac{\partial \delta^{*}}{\partial \mathcal{L}}>0, \frac{\partial \delta^{*}}{\partial \beta}<0$, and $\frac{\partial \delta^{*}}{\partial \gamma_{f}}<0 . \square$

Proof of proposition 3: In equilibrium, the incentive constraint of the bank binds:

$$
\gamma=\frac{1-k(\delta)}{k(\delta)(\beta-1)-(\alpha-1)}
$$

Substituting $\gamma$ into the objective of the regulator, we can write $(P)$ as

$$
\max _{\delta} \Sigma_{b}(\delta) \equiv(q \alpha+(1-q)(F(\tau(\delta)) \mathcal{L}+k(\delta) \beta)-1) \frac{1-k(\delta)}{k(\delta)(\beta-1)-(\alpha-1)} E
$$


Taking the first-order condition with respect to $\delta$, we get

$$
\begin{aligned}
(k(\delta)(\beta-1)-(\alpha-1))( & -k^{\prime}(\delta)(q \alpha+(1-q)(F(\tau(\delta)) \mathcal{L}+k(\delta) \beta)-1) \\
& \left.+(1-k(\delta))\left((1-q)\left(f(\tau(\delta)) \mathcal{L} \tau^{\prime}(\delta)+k^{\prime}(\delta) \beta\right)\right)\right) \\
& \quad-(\beta-1) k^{\prime}(\delta)(q \alpha+(1-q)(F(\tau(\delta)) \mathcal{L}+k(\delta) \beta)-1)(1-k(\delta))=0,
\end{aligned}
$$

which is equivalent to

$$
\begin{aligned}
&-(\beta-\alpha)(q \alpha+(1-q)(F(\tau(\delta)) \mathcal{L}+k(\delta) \beta)-1) k^{\prime}(\delta) \\
&+(k(\delta)(\beta-1)-(\alpha-1))(1-k(\delta))\left((1-q)\left(f(\tau(\delta)) \tau^{\prime}(\delta) \mathcal{L}+k^{\prime}(\delta) \beta\right)\right)=0 .
\end{aligned}
$$

Defining the function $H(\delta)$ as the left-hand side of (6.3), we can rewrite the first-order condition as $H(\delta)=0$.

Let us derive the conditions under which $H(1)<0$, or equivalently, the conditions under which $\delta^{*}<1$. Evaluating the first-order condition at $\delta=1$, we get

$$
\begin{aligned}
H(1)= & -\left(q \alpha+(1-q)\left(F\left(\frac{\mathcal{L}}{\beta}\right) \mathcal{L}+k(1) \beta\right)-1\right)(\beta-\alpha)\left(\int_{\frac{\mathcal{L}}{\beta}}^{1}\left(p-p_{m}\right) f(p) d p-\frac{\mathcal{L}}{\beta} \tau^{\prime}(1) f\left(\frac{\mathcal{L}}{\beta}\right)\right) \\
& +(k(1)(\beta-1)-(\alpha-1))(1-k(1))(1-q) \int_{\frac{\mathcal{L}}{\beta}}^{1}\left(p-p_{m}\right) f(p) d p \beta .
\end{aligned}
$$

Full precision $\delta^{*}=1$ cannot be a solution to $(P)$ if $H(1)<0$. Hence, if $k(1)(\beta-1)-(\alpha-1)$ is sufficiently small, we have $H(1)<0$. Further, it is readily seen that $H(1)$ is decreasing in $q$. Hence, we have $H(1)<0$ if $q$ is sufficiently large. Finally,

$$
\begin{aligned}
\frac{\partial H(1)}{\partial \mathcal{L}}= & -(\beta-1)(q \alpha+(1-q)(F(\tau(1)) \mathcal{L}+k(1) \beta)-1) \\
& \times\left[-\left(\tau(1)-p_{m}\right) f(\tau(1)) \frac{1}{\beta}+\left(\frac{f^{\prime}(\tau(1))}{\beta} \frac{\mathcal{L}}{\beta}\left(\frac{\mathcal{L}}{\beta}-p_{m}\right)+f(\tau(1))\left(2 \frac{\mathcal{L}}{\beta^{2}}-\frac{p_{m}}{\beta}\right)\right)\right] \\
& -(\beta-1)\left(\int_{\tau(1)}^{1}\left(p-p_{m}\right) f(p) d p+f(\tau(1)) \frac{\mathcal{L}}{\beta}\left(\frac{\mathcal{L}}{\beta}-p_{m}\right)\right) \\
& \times\left[(1-q) F(\tau(1))+(1-q) \frac{\mathcal{L} f(\tau(1))}{\beta}-(1-q) \beta \tau(1) \frac{f(\tau(1))}{\beta}\right] \\
& -\tau(1) \frac{f(\tau(1))}{\beta}((\beta-1)+(\alpha-1)-2 k(1)(\beta-1))(1-q) \beta \int_{\tau(1)}^{1}\left(p-p_{m}\right) f(p) d p \\
& -(k(1)(\beta-1)-(\alpha-1))(1-k(1))(1-q) \beta\left(\tau(1)-p_{m}\right) \frac{f(\tau(1))}{\beta},
\end{aligned}
$$


Hence, if $(\beta-1)+(\alpha-1)-2 k(1)(\beta-1)=(\beta-1)+(\alpha-1)-2 \int_{\frac{\mathcal{L}}{\beta}}^{1} p f(p) d p(\beta-1) \geq 0$ and $f^{\prime}\left(\frac{\mathcal{L}}{\beta}\right) \geq 0$ or $f^{\prime}\left(\frac{\mathcal{L}}{\beta}\right)$ sufficiently close to $0, H(1)$ is decreasing in $\mathcal{L}$; as a result, $H(1)<0$ if $\mathcal{L}$ is sufficiently large.

Proof of corollary 2: Given that $\delta^{*}$ maximizes $\Sigma_{b}$, it must $H^{\prime}\left(\delta^{*}\right) \leq 0$. For comparative statics, we also need to assume that the global maximum is unique and regular $H^{\prime}\left(\delta^{*}\right) \neq 0$, which is a generic condition and guaranteed if $(P)$ is a convex program $\left(H^{\prime}<0\right)$. It then follows that the comparative static of $\delta^{*}$ in a variable $x$ has the sign of $\partial H / \partial x$. First, we have

$$
\begin{aligned}
\frac{\partial H\left(\delta^{*}\right)}{\partial \alpha}= & -q(\beta-\alpha) k^{\prime}\left(\delta^{*}\right)+\left(q \alpha+(1-q)\left(F\left(\tau\left(\delta^{*}\right)\right) \mathcal{L}+k\left(\delta^{*}\right) \beta\right)-1\right) k^{\prime}\left(\delta^{*}\right) \\
& -\left(1-k\left(\delta^{*}\right)(1-q) \beta \int_{\tau\left(\delta^{*}\right)}^{1}\left(p-p_{m}\right) f(p) d p\right.
\end{aligned}
$$

From (6.3), we know that

$$
\begin{aligned}
H\left(\delta^{*}\right)=-(\beta-\alpha)(q \alpha & \left.+(1-q)\left(F\left(\tau\left(\delta^{*}\right)\right) \mathcal{L}+k\left(\delta^{*}\right) \beta\right)-1\right) k^{\prime}\left(\delta^{*}\right) \\
& +\left(k\left(\delta^{*}\right)(\beta-1)-(\alpha-1)\right)\left(1-k\left(\delta^{*}\right)\right) \beta(1-q) \int_{\tau\left(\delta^{*}\right)}^{1}\left(p-p_{m}\right) f(p) d p=0 .
\end{aligned}
$$

As a result,

$$
\begin{aligned}
\frac{\partial H\left(\delta^{*}\right)}{\partial \alpha}= & -q(\beta-\alpha) k^{\prime}\left(\delta^{*}\right)+\frac{k\left(\delta^{*}\right)(\beta-1)-(\alpha-1)}{\beta-\alpha}\left(1-k\left(\delta^{*}\right)\right) \beta(1-q) \int_{\tau\left(\delta^{*}\right)}^{1}\left(p-p_{m}\right) f(p) d p \\
& -\left(1-k\left(\delta^{*}\right)(1-q) \beta \int_{\tau\left(\delta^{*}\right)}^{1}\left(p-p_{m}\right) f(p) d p\right. \\
= & -q(\beta-\alpha) k^{\prime}\left(\delta^{*}\right)-\frac{(\beta-1)\left(1-k\left(\delta^{*}\right)\right)}{\beta-\alpha}\left(1-k\left(\delta^{*}\right)\right) \beta(1-q) \int_{\tau\left(\delta^{*}\right)}^{1}\left(p-p_{m}\right) f(p) d p<0 .
\end{aligned}
$$

Next, let us derive the partial derivative of $H$ with respect to $\mathcal{L}$. We have

$$
\begin{aligned}
\frac{\partial H\left(\delta^{*}\right)}{\partial \mathcal{L}}= & -(\beta-\alpha)\left(q \alpha+(1-q)\left(F\left(\tau\left(\delta^{*}\right)\right) \mathcal{L}+k\left(\delta^{*}\right) \beta\right)-1\right) \\
& \times\left[-\left(\tau(\delta)-p_{m}\right) f\left(\tau\left(\delta^{*}\right)\right) \frac{1}{\delta^{*} \beta}+\frac{1}{\left(\delta^{*}\right)^{2}}\left(\frac{f^{\prime}\left(\tau\left(\delta^{*}\right)\right)}{\delta^{*} \beta} \frac{\mathcal{L}}{\beta}\left(\frac{\mathcal{L}}{\beta}-p_{m}\right)+f\left(\tau\left(\delta^{*}\right)\right)\left(2 \frac{\mathcal{L}}{\beta^{2}}-\frac{p_{m}}{\beta}\right)\right)\right] \\
& -(\beta-\alpha)\left(\int_{\tau\left(\delta^{*}\right)}^{1}\left(p-p_{m}\right) f(p) d p+\frac{f\left(\tau\left(\delta^{*}\right)\right)}{\left(\delta^{*}\right)^{2}} \frac{\mathcal{L}}{\beta}\left(\frac{\mathcal{L}}{\beta}-p_{m}\right)\right) \\
& \times\left[(1-q) F\left(\tau\left(\delta^{*}\right)\right)+(1-q) \frac{\mathcal{L} f\left(\tau\left(\delta^{*}\right)\right)}{\delta^{*} \beta}-(1-q) \beta\left(\delta^{*} \tau\left(\delta^{*}\right)+\left(1-\delta^{*}\right) p_{m}\right) \frac{f\left(\tau\left(\delta^{*}\right)\right)}{\delta^{*} \beta}\right] \\
& -\left(\delta^{*} \tau(\delta)+\left(1-\delta^{*}\right) p_{m}\right) \frac{f\left(\tau\left(\delta^{*}\right)\right)}{\delta^{*} \beta}\left((\beta-1)+(\alpha-1)-2 k\left(\delta^{*}\right)(\beta-1)\right)(1-q) \beta \int_{\tau\left(\delta^{*}\right)}^{1}\left(p-p_{m}\right) f(p) d p \\
& -\left(k\left(\delta^{*}\right)(\beta-1)-(\alpha-1)\right)\left(1-k\left(\delta^{*}\right)\right)(1-q) \beta\left(\tau\left(\delta^{*}\right)-p_{m}\right) \frac{f\left(\tau\left(\delta^{*}\right)\right)}{\delta^{*} \beta} .
\end{aligned}
$$


We can rewrite $\frac{\partial H\left(\delta^{*}\right)}{\partial \mathcal{L}}$ as

$$
\begin{aligned}
\frac{\partial H\left(\delta^{*}\right)}{\partial \mathcal{L}}= & -\overbrace{(\beta-\alpha)\left(q \alpha+(1-q)\left(F\left(\tau\left(\delta^{*}\right)\right) \mathcal{L}+k\left(\delta^{*}\right) \beta\right)-1\right)}^{>0} \\
& \times \frac{1}{\left(\delta^{*}\right)^{2}}\left[\frac{f\left(\tau\left(\delta^{*}\right)\right)}{\beta}\left(\frac{\mathcal{L}}{\beta}\right)+\left(\frac{f^{\prime}\left(\tau\left(\delta^{*}\right)\right)}{\delta^{*} \beta} \frac{\mathcal{L}}{\beta}\left(\frac{\mathcal{L}}{\beta}-p_{m}\right)\right]\right. \\
& -\overbrace{(\beta-\alpha)\left(\int_{\tau\left(\delta^{*}\right)}^{1}\left(p-p_{m}\right) f(p) d p+f\left(\tau\left(\delta^{*}\right)\right) \frac{\mathcal{L}}{\left(\delta^{*}\right)^{2} \beta}\left(\frac{\mathcal{L}}{\beta}-p_{m}\right)\right)}^{>0} \\
& \times \overbrace{\left[(1-q) F\left(\tau\left(\delta^{*}\right)\right)+(1-q) \frac{\mathcal{L} f\left(\tau\left(\delta^{*}\right)\right)}{\delta^{*} \beta}-(1-q) \beta\left(\delta^{*} \tau\left(\delta^{*}\right)+\left(1-\delta^{*}\right) p_{m}\right) \frac{f\left(\tau\left(\delta^{*}\right)\right)}{\delta^{*} \beta}\right]}^{\left(\delta^{*} \tau\left(\delta^{*}\right)+\left(1-\delta^{*}\right) p_{m}\right) \frac{f\left(\tau\left(\delta^{*}\right)\right)}{\delta^{*} \beta}\left((\beta-1)+(\alpha-1)-2 k\left(\delta^{*}\right)(\beta-1)\right)(1-q) \beta \int_{\tau\left(\delta^{*}\right)}^{1}}] \\
& -\overbrace{\left(k\left(\delta^{*}\right)(\beta-1)-(\alpha-1)\right)\left(1-k\left(\delta^{*}\right)\right)(1-q) \beta\left(\tau\left(\delta^{*}\right)-p_{m}\right) \frac{f\left(\tau\left(\delta^{*}\right)\right)}{\delta^{*} \beta}}^{>0} f(p) d p
\end{aligned}
$$

Hence, if $(\beta-1)+(\alpha-1)-2 k(1)(\beta-1)=(\beta-1)+(\alpha-1)-2 \int_{\frac{\mathcal{L}}{\beta}}^{1} p f(p) d p(\beta-1) \geq 0$, then

$$
(\beta-1)+(\alpha-1)-2 \int_{\tau\left(\delta^{*}\right)}^{1}\left(\delta^{*} p+\left(1-\delta^{*}\right) p_{m}\right) f(p) d p(\beta-1) \geq(\beta-1)+(\alpha-1)-2 \int_{\frac{\mathcal{L}}{\beta}}^{1} p f(p) d p(\beta-1) \geq 0
$$

which implies that

$$
-\left(\delta^{*} \tau\left(\delta^{*}\right)+\left(1-\delta^{*}\right) p_{m}\right) \frac{f\left(\tau\left(\delta^{*}\right)\right)}{\delta^{*} \beta}\left((\beta-1)+(\alpha-1)-2 k\left(\delta^{*}\right)(\beta-1)\right)(1-q) \beta \int_{\tau\left(\delta^{*}\right)}^{1}\left(p-p_{m}\right) f(p) d p<0
$$

Similarly, if $f^{\prime}\left(\tau\left(\delta^{*}\right)\right) \geq 0$ or $f^{\prime}\left(\tau\left(\delta^{*}\right)\right)$ sufficiently close to 0 , then

$$
\frac{1}{\left(\delta^{*}\right)^{2}}\left[\frac{f\left(\tau\left(\delta^{*}\right)\right)}{\beta}\left(\frac{\mathcal{L}}{\beta}\right)+\left(\frac{f^{\prime}\left(\tau\left(\delta^{*}\right)\right)}{\delta^{*} \beta} \frac{\mathcal{L}}{\beta}\left(\frac{\mathcal{L}}{\beta}-p_{m}\right)\right]>0\right.
$$

As a result, if $(\beta-1)+(\alpha-1)-2 k(1)(\beta-1) \geq 0$ and $f^{\prime}\left(\tau\left(\delta^{*}\right)\right) \geq 0$ or $f^{\prime}\left(\tau\left(\delta^{*}\right)\right)$ sufficiently close to 0 , then $\frac{\partial H\left(\delta^{*}\right)}{\partial \mathcal{L}}<0$.

We now derive the partial derivative of $H$ with respect to $q$. We have

$$
\begin{aligned}
\frac{\partial H\left(\delta^{*}\right)}{\partial q}= & -(\beta-\alpha)\left(q \alpha+(1-q)\left(F\left(\tau\left(\delta^{*}\right)\right) \mathcal{L}+k\left(\delta^{*}\right) \beta\right)-1\right) \\
& -(\beta-\alpha)\left(\alpha-\left(F\left(\tau\left(\delta^{*}\right)\right) \mathcal{L}+k\left(\delta^{*}\right) \beta\right)\right) k^{\prime}\left(\delta^{*}\right) \\
& -\left(k\left(\delta^{*}\right)(\beta-1)-(\alpha-1)\right)\left(1-k\left(\delta^{*}\right)\right) \beta \int_{\tau\left(\delta^{*}\right)}^{1}\left(p-p_{m}\right) f(p) d p<0 .
\end{aligned}
$$


Next, we derive the comparative statics on the optimal capital requirement $\gamma$. Note that

$$
\frac{\partial \tau\left(\delta^{*}\right)}{\partial \alpha}=-\frac{\partial \delta^{*}}{\partial \alpha}\left(\frac{\mathcal{L}}{\beta}-p_{m}\right)
$$

As a result,

$$
\frac{\partial k\left(\delta^{*}\right)}{\partial \alpha}=\frac{\partial \delta^{*}}{\partial \alpha} \int_{\tau\left(\delta^{*}\right)}^{1}\left(p-p_{m}\right) f(p) d p+\left(\delta^{*} \tau\left(\delta^{*}\right)+\left(1-\delta^{*}\right) p_{m}\right) f\left(\tau\left(\delta^{*}\right)\right)\left(\frac{\frac{\partial \delta^{*}}{\partial \alpha}}{\left(\delta^{*}\right)^{2}}\left(\frac{\mathcal{L}}{\beta}-p_{m}\right)\right) .
$$

Taking the derivative of

$$
\gamma^{*}=\frac{1-k\left(\delta^{*}\right)}{k\left(\delta^{*}\right)(\beta-1)-(\alpha-1)}
$$

with respect to $\alpha$ yields

$$
\frac{\partial \gamma^{*}}{\partial \alpha}=\frac{-\frac{\partial k\left(\delta^{*}\right)}{\partial \alpha}\left(k\left(\delta^{*}\right)(\beta-1)-(\alpha-1)\right)-\left(1-k\left(\delta^{*}\right)\right) \frac{\partial k\left(\delta^{*}\right)}{\partial \alpha}(\beta-1)}{\left(k\left(\delta^{*}\right)(\beta-1)-(\alpha-1)\right)^{2}} .
$$

Hence, $\frac{\partial \delta^{*}}{\partial \alpha}<0$ implies $\frac{\partial \gamma^{*}}{\partial \alpha}>0$.

Second, note that

$$
\frac{\partial \tau\left(\delta^{*}\right)}{\partial \mathcal{L}}=\frac{1}{\delta^{*} \beta}-\frac{\frac{\partial \delta_{2}^{*}}{\partial \mathcal{L}}}{\left(\delta^{*}\right)^{2}}\left(\frac{\mathcal{L}}{\beta}-p_{m}\right)
$$

As a result,

$$
\frac{\partial k\left(\delta^{*}\right)}{\partial \mathcal{L}}=\frac{\partial \delta^{*}}{\partial \mathcal{L}} \int_{\tau\left(\delta^{*}\right)}^{1}\left(p-p_{m}\right) f(p) d p+\left(\delta^{*} \tau\left(\delta^{*}\right)+\left(1-\delta^{*}\right) p_{m}\right) f\left(\tau\left(\delta^{*}\right)\right)\left(-\frac{1}{\delta^{*} \beta}+\frac{\frac{\partial \delta^{*}}{\partial \mathcal{L}}}{\left(\delta^{*}\right)^{2}}\left(\frac{\mathcal{L}}{\beta}-p_{m}\right)\right) .
$$

Taking the derivative of

$$
\gamma^{*}=\frac{1-k\left(\delta^{*}\right)}{k\left(\delta^{*}\right)(\beta-1)-(\alpha-1)}
$$

with respect to $\mathcal{L}$ yields

$$
\frac{\partial \gamma^{*}}{\partial \mathcal{L}}=\frac{-\frac{\partial k\left(\delta^{*}\right)}{\partial \mathcal{L}}\left(k\left(\delta^{*}\right)(\beta-1)-(\alpha-1)\right)-\left(1-k\left(\delta^{*}\right)\right) \frac{\partial k\left(\delta^{*}\right)}{\partial \mathcal{L}}(\beta-1)}{\left(k\left(\delta^{*}\right)(\beta-1)-(\alpha-1)\right)^{2}} .
$$

Hence, $\frac{\partial \delta^{*}}{\partial \mathcal{L}}<0$ implies $\frac{\partial \gamma^{*}}{\partial \mathcal{L}}>0$.

Third, note that

$$
\frac{\partial \tau\left(\delta^{*}\right)}{\partial q}=-\frac{\frac{\partial \delta_{2}^{*}}{\partial q}}{\left(\delta^{*}\right)^{2}}\left(\frac{\mathcal{L}}{\beta}-p_{m}\right) .
$$


As a result,

$$
\frac{\partial k\left(\delta^{*}\right)}{\partial q}=\frac{\partial \delta^{*}}{\partial q} \int_{\tau\left(\delta^{*}\right)}^{1}\left(p-p_{m}\right) f(p) d p+\left(\delta^{*} \tau\left(\delta^{*}\right)+\left(1-\delta^{*}\right) p_{m}\right) f\left(\tau\left(\delta^{*}\right)\right) \frac{\frac{\partial \delta^{*}}{\partial q}}{\left(\delta^{*}\right)^{2}}\left(\frac{\mathcal{L}}{\beta}-p_{m}\right)
$$

Taking the derivative of

$$
\gamma^{*}=\frac{1-k\left(\delta^{*}\right)}{k\left(\delta^{*}\right)(\beta-1)-(\alpha-1)}
$$

with respect to $q$ yields

$$
\frac{\partial \gamma^{*}}{\partial q}=\frac{\left.-\frac{\partial k\left(\delta^{*}\right)}{\partial q}\left(k\left(\delta^{*}\right)(\beta-1)-(\alpha-1)\right)-\left(1-k\left(\delta^{*}\right)\right) \frac{\partial k\left(\delta^{*}\right)}{\partial q}(\beta-1)\right)}{\left(k\left(\delta^{*}\right)(\beta-1)-(\alpha-1)\right)^{2}} .
$$

Hence, $\frac{\partial \delta^{*}}{\partial q}<0$ implies $\frac{\partial k\left(\delta^{*}\right)}{\partial q}<0$, which implies $\frac{\partial \gamma^{*}}{\partial q}>0$.

Finally, we derive the comparative statics with respect to $\beta$. Note that

$$
\frac{\partial \tau\left(\delta^{*}\right)}{\partial \beta}=-\frac{\mathcal{L}}{\delta^{*} \beta^{2}}-\frac{\frac{\partial \delta^{*}}{\partial \beta}}{\left(\delta^{*}\right)^{2}}\left(\frac{\mathcal{L}}{\beta}-p_{m}\right)
$$

As a result,

$$
\frac{\partial k\left(\delta^{*}\right)}{\partial \beta}=\frac{\partial \delta^{*}}{\partial \beta} \int_{\tau\left(\delta^{*}\right)}^{1}\left(p-p_{m}\right) f(p) d p+\left(\delta^{*} \tau\left(\delta^{*}\right)+\left(1-\delta^{*}\right) p_{m}\right) f\left(\tau\left(\delta^{*}\right)\right)\left(\frac{\mathcal{L}}{\delta^{*} \beta^{2}}+\frac{\frac{\partial \delta^{*}}{\partial \beta}}{\left(\delta^{*}\right)^{2}}\left(\frac{\mathcal{L}}{\beta}-p_{m}\right)\right)
$$

Taking the derivative of

$$
\gamma^{*}=\frac{1-k\left(\delta^{*}\right)}{k\left(\delta^{*}\right)(\beta-1)-(\alpha-1)}
$$

with respect to $\beta$ yields

$$
\frac{\partial \gamma^{*}}{\partial \beta}=\frac{-\frac{\partial k\left(\delta^{*}\right)}{\partial \beta}\left(k\left(\delta^{*}\right)(\beta-1)-(\alpha-1)\right)-\left(1-k\left(\delta^{*}\right)\right)\left(k\left(\delta^{*}\right)+\frac{\partial k\left(\delta^{*}\right)}{\partial \beta}(\beta-1)\right)}{\left(k\left(\delta^{*}\right)(\beta-1)-(\alpha-1)\right)^{2}}
$$

which is equivalent to

$$
\frac{\partial \gamma^{*}}{\partial \beta}=-\frac{\left(1-k\left(\delta^{*}\right)\right) k\left(\delta^{*}\right)+(\beta-\alpha) \frac{\partial k\left(\delta^{*}\right)}{\partial \beta}}{\left(k\left(\delta^{*}\right)(\beta-1)-(\alpha-1)\right)^{2}}
$$

Note that $\frac{\partial \delta^{*}}{\partial \beta}>0$ and (6.5) imply that $\frac{\partial k\left(\delta^{*}\right)}{\partial \beta}>0$. Further, $\frac{\partial k\left(\delta^{*}\right)}{\partial \beta}>0$ and (6.6) imply that $\frac{\partial \gamma^{*}}{\partial \beta}<0$. Hence, $\frac{\partial \delta^{*}}{\partial \beta}>0$ implies that $\frac{\partial \gamma^{*}}{\partial \beta}<0$. Further, we know that the optimal capital requirement is given by

$$
\frac{\gamma^{*} \alpha-\left(\gamma^{*}-1\right)}{\gamma^{*} \beta-\left(\gamma^{*}-1\right)}=k\left(\delta^{*}\right)
$$


Taking the derivative of the left-hand side with respect to beta yields

$$
\left(\gamma^{*} \beta-\left(\gamma^{*}-1\right)\right) \frac{(\alpha-1) \frac{\partial \gamma^{*}}{\partial \beta}}{\left(\gamma^{*} \beta-\left(\gamma^{*}-1\right)\right)^{2}}-\left(\gamma^{*} \alpha-\left(\gamma^{*}-1\right)\right) \frac{\gamma^{*}+(\beta-1) \frac{\partial \gamma^{*}}{\partial \beta}}{\left(\gamma^{*} \beta-\left(\gamma^{*}-1\right)\right)^{2}}
$$

Suppose that $\frac{\partial \gamma^{*}}{\partial \beta}>0$. This implies that the left-hand side of (6.7) decreases in $\beta$, which in turn implies that $k\left(\delta^{*}\right)$ decreases in $\beta$, which is equivalent to

$$
\frac{\partial k\left(\delta^{*}\right)}{\partial \beta}=\frac{\partial \delta^{*}}{\partial \beta} \int_{\tau\left(\delta^{*}\right)}^{1}\left(p-p_{m}\right) f(p) d p+\left(\delta^{*} \tau\left(\delta^{*}\right)+\left(1-\delta^{*}\right) p_{m}\right) f\left(\tau\left(\delta^{*}\right)\right)\left(\frac{\mathcal{L}}{\delta^{*} \beta^{2}}+\frac{\frac{\partial \delta^{*}}{\partial \beta}}{\left(\delta^{*}\right)^{2}}\left(\frac{\mathcal{L}}{\beta}-p_{m}\right)\right)<0
$$

As a result, $\frac{\partial \delta^{*}}{\partial \beta}<0$. Hence, $\frac{\partial \gamma^{*}}{\partial \beta}>0$ implies that $\frac{\partial \delta^{*}}{\partial \beta}<0 . \square$

\section{Proof of proposition 4:}

In equilibrium, as the participation constraint of the bad bank binds, the optimal capital requirement is

$$
\gamma=\frac{1-k(\delta)}{k(\delta)(\beta-1)}
$$

As a result, the regulator's maximization problem becomes

$$
\max _{\delta} \Sigma_{b}(\delta) \equiv \frac{1}{2}(q \alpha+(1-q)(F(\tau(\delta)) \mathcal{L}+k(\delta) \beta)-1) \frac{1-k(\delta)}{k(\delta)(\beta-1)} E
$$

Taking the first-order condition of the optimization problem, we get

$$
\begin{aligned}
&(\beta-1) k(\delta)\left(-k^{\prime}(\delta) E(\right.q \alpha+(1-q)(F(\tau(\delta)) \mathcal{L}+k(\delta) \beta)-1) \\
&\left.+(E-k(\delta) E)\left((1-q)\left(f(\tau(\delta)) \mathcal{L} \tau^{\prime}(\delta)+k^{\prime}(\delta) \beta\right)\right)\right) \\
& \quad-(\beta-1) k^{\prime}(\delta)(q \alpha+(1-q)(F(\tau(\delta)) \mathcal{L}+k(\delta) \beta)-1)(E-k(\delta) E)=0,
\end{aligned}
$$

which is equivalent to

$$
-(q \alpha+(1-q)(F(\tau(\delta)) \mathcal{L}+k(\delta) \beta)-1) k^{\prime}(\delta)+k(\delta)(1-k(\delta))\left((1-q)\left(f(\tau(\delta)) \mathcal{L} \tau^{\prime}(\delta)+k^{\prime}(\delta) \beta\right)\right)=0
$$

Let us define the left-hand side of $(6.8)$ as $H(\delta)$. The first-order condition with respect to $\delta$ can then be rewritten as $H(\delta)=0$. 
Next, let us derive the conditions under which $H(1)<0$. We know that $\tau(1)=\frac{\mathcal{L}}{\beta}$. Hence, we have

$$
\begin{array}{r}
H(1)=-\left(q \alpha+(1-q)\left(F\left(\frac{\mathcal{L}}{\beta}\right) \mathcal{L}+k(1) \beta\right)-1\right)\left(\int_{\frac{\mathcal{L}}{\beta}}^{1}\left(p-p_{m}\right) f(p) d p-\frac{\mathcal{L}}{\beta} \tau^{\prime}(1) f\left(\frac{\mathcal{L}}{\beta}\right)\right) \\
+(1-k(1)) k(1)(1-q) \beta \int_{\frac{\mathcal{L}}{\beta}}^{1}\left(p-p_{m}\right) f(p) d p .
\end{array}
$$

As a result, $H(1)<0$ if and only if

$$
\begin{aligned}
\left(q \alpha+(1-q)\left(F\left(\frac{\mathcal{L}}{\beta}\right) \mathcal{L}+k(1) \beta\right)-1\right)\left(\int_{\frac{\mathcal{L}}{\beta}}^{1}\left(p-p_{m}\right) f(p) d p-\frac{\mathcal{L}}{\beta} \tau^{\prime}(1) f\left(\frac{\mathcal{L}}{\beta}\right)\right) & \\
& >(1-k(1)) k(1)(1-q) \beta \int_{\frac{\mathcal{L}}{\beta}}^{1}\left(p-p_{m}\right) f(p) d p .
\end{aligned}
$$

Condition (6.9) is satisfied if $\alpha$ is sufficiently large. Further, condition (6.9) can be rewritten as

$$
\begin{array}{r}
\left(q \alpha+(1-q)\left(F\left(\frac{\mathcal{L}}{\beta}\right) \mathcal{L}+\int_{\frac{\mathcal{L}}{\beta}}^{1} p f(p) d p \beta\right)-1\right)\left(\int_{\frac{\mathcal{L}}{\beta}}^{1}\left(p-p_{m}\right) f(p) d p+\frac{\mathcal{L}}{\beta}\left(\frac{\mathcal{L}}{\beta}-p_{m}\right) f\left(\frac{\mathcal{L}}{\beta}\right)\right) \\
>\left(1-\int_{\frac{\mathcal{L}}{\beta}}^{1} p f(p) d p\right) \int_{\frac{\mathcal{L}}{\beta}}^{1} p f(p) d p(1-q) \beta \int_{\frac{\mathcal{L}}{\beta}}^{1}\left(p-p_{m}\right) f(p) d p .
\end{array}
$$

Condition (6.10) is satisfied if $q$ is sufficiently large. Finally, if $1-2 k(1)=1-2 \int_{\frac{\mathcal{L}}{\beta}}^{1} p f(p) d p \geq 0$ and $f^{\prime}\left(\frac{\mathcal{L}}{\beta}\right) \geq 0$ or $f^{\prime}\left(\frac{\mathcal{L}}{\beta}\right)$ sufficiently close to 0 , then the right-hand side of $(6.9)$ decreases in $\mathcal{L}$ whereas the left-hand side of $(6.9)$ increases in $\mathcal{L}$. As a result, $H(1)<0$ if $\mathcal{L}$ is sufficiently large.

Next, let us derive the comparative statics on $\delta^{*}$. For comparative statics, we also need to assume that the global maximum is unique and regular $H^{\prime}\left(\delta^{*}\right) \neq 0$, which is a generic condition and guaranteed if $(P)$ is a convex program $\left(H^{\prime}<0\right)$. It then follows that the comparative static of $\delta^{*}$ in a variable $X$ has the sign of $\partial H / \partial X$, which we conduct next. First, we have

$$
\frac{\partial H\left(\delta^{*}\right)}{\partial \alpha}=-q k^{\prime}\left(\delta^{*}\right)<0
$$


Second, we have

$$
\begin{aligned}
& \frac{\partial H\left(\delta^{*}\right)}{\partial \mathcal{L}}=-\overbrace{\left(q \alpha+(1-q)\left(F\left(\tau\left(\delta^{*}\right)\right) \mathcal{L}+k\left(\delta^{*}\right) \beta\right)-1\right)}^{>0} \frac{1}{\left(\delta^{*}\right)^{2}}\left[\frac{f\left(\tau\left(\delta^{*}\right)\right)}{\beta}\left(\frac{\mathcal{L}}{\beta}\right)+\left(\frac{f^{\prime}\left(\tau\left(\delta^{*}\right)\right)}{\delta^{*} \beta} \frac{\mathcal{L}}{\beta}\left(\frac{\mathcal{L}}{\beta}-p_{m}\right)\right]\right. \\
& -\overbrace{\left(\int_{\tau\left(\delta^{*}\right)}^{1}\left(p-p_{m}\right) f(p) d p+f\left(\tau\left(\delta^{*}\right)\right) \frac{\mathcal{L}}{\left(\delta^{*}\right)^{2} \beta}\left(\frac{\mathcal{L}}{\beta}-p_{m}\right)\right)}^{>0} \\
& \times \overbrace{\left[(1-q) F\left(\tau\left(\delta^{*}\right)\right)+(1-q) \frac{\mathcal{L} f\left(\tau\left(\delta^{*}\right)\right)}{\delta^{*} \beta}-(1-q) \beta\left(\delta^{*} \tau\left(\delta^{*}\right)+\left(1-\delta^{*}\right) p_{m}\right) \frac{f\left(\tau\left(\delta^{*}\right)\right)}{\delta^{*} \beta}\right]}^{>0} \\
& -\left(\delta^{*} \tau\left(\delta^{*}\right)+\left(1-\delta^{*}\right) p_{m}\right) \frac{f\left(\tau\left(\delta^{*}\right)\right)}{\delta^{*} \beta}\left(1-2 k\left(\delta^{*}\right)\right)(1-q) \beta \int_{\tau\left(\delta^{*}\right)}^{1}\left(p-p_{m}\right) f(p) d p \\
& -\overbrace{k\left(\delta^{*}\right)\left(1-k\left(\delta^{*}\right)\right)(1-q) \beta\left(\tau\left(\delta^{*}\right)-p_{m}\right) \frac{f\left(\tau\left(\delta^{*}\right)\right)}{\delta^{*} \beta}}^{>0} .
\end{aligned}
$$

Hence, if $1-2 k\left(\delta^{*}\right) \geq 1-2 k(1) \geq 0$ and $f^{\prime}\left(\tau\left(\delta^{*}\right)\right) \geq 0$ or $f^{\prime}\left(\tau\left(\delta^{*}\right)\right)$ close to 0 , then $\frac{\partial H\left(\delta^{*}\right)}{\partial \mathcal{L}}<0$. Third, we have

$$
\frac{\partial H\left(\delta^{*}\right)}{\partial q}=-\left(\alpha-\left(F\left(\tau\left(\delta^{*}\right)\right) \mathcal{L}+k\left(\delta^{*}\right) \beta\right)\right) k^{\prime}\left(\delta^{*}\right)-k\left(\delta^{*}\right)\left(1-k\left(\delta^{*}\right)\right) \beta \int_{\tau\left(\delta^{*}\right)}^{1}\left(p-p_{m}\right) f(p) d p<0
$$

As a result, $\frac{\partial H\left(\delta^{*}\right)}{\partial q}<0$.

Next, let us now derive the comparative statics on the optimal capital requirement $\gamma^{*}$. First, note that

$$
\frac{\partial \tau\left(\delta^{*}\right)}{\partial \alpha}=-\frac{2 \frac{\partial \delta^{*}}{\partial \alpha}}{\delta^{*}}\left(\frac{\mathcal{L}}{\beta}-p_{m}\right)
$$

As a result,

$$
\frac{\partial k\left(\delta^{*}\right)}{\partial \alpha}=\frac{\partial \delta^{*}}{\partial \alpha} \int_{\tau\left(\delta^{*}\right)}^{1}\left(p-p_{m}\right) f(p) d p+\left(\delta^{*} \tau\left(\delta^{*}\right)+\left(1-\delta^{*}\right) p_{m}\right) f\left(\tau\left(\delta^{*}\right)\right)\left(\frac{2 \frac{\partial \delta^{*}}{\partial \alpha}}{\delta^{*}}\left(\frac{\mathcal{L}}{\beta}-p_{m}\right)\right) .
$$

Taking the derivative of

$$
\gamma^{*}=\frac{1-k\left(\delta^{*}\right)}{k\left(\delta^{*}\right)(\beta-1)}
$$

with respect to $\alpha$ yields

$$
\frac{\partial \gamma^{*}}{\partial \alpha}=\frac{-\frac{\partial k\left(\delta^{*}\right)}{\partial \alpha} k\left(\delta^{*}\right)(\beta-1)-\left(1-k\left(\delta^{*}\right)\right) \frac{\partial k\left(\delta^{*}\right)}{\partial \alpha}(\beta-1)}{\left(k\left(\delta^{*}\right)(\beta-1)\right)^{2}}
$$

Hence, $\frac{\partial \delta^{*}}{\partial \alpha}<0$ implies $\frac{\partial \gamma^{*}}{\partial \alpha}>0$. 
Second, note that

$$
\frac{\partial \tau\left(\delta^{*}\right)}{\partial \mathcal{L}}=\frac{1}{\delta^{*} \beta}-\frac{2 \frac{\partial \delta^{*}}{\partial \mathcal{L}}}{\delta^{*}}\left(\frac{\mathcal{L}}{\beta}-p_{m}\right)
$$

As a result,

$$
\frac{\partial k\left(\delta^{*}\right)}{\partial \mathcal{L}}=\frac{\partial \delta^{*}}{\partial \mathcal{L}} \int_{\tau\left(\delta^{*}\right)}^{1}\left(p-p_{m}\right) f(p) d p+\left(\delta^{*} \tau\left(\delta^{*}\right)+\left(1-\delta^{*}\right) p_{m}\right) f\left(\tau\left(\delta^{*}\right)\right)\left(-\frac{1}{\delta^{*} \beta}+\frac{2 \frac{\partial \delta^{*}}{\partial \mathcal{L}}}{\delta^{*}}\left(\frac{\mathcal{L}}{\beta}-p_{m}\right)\right) .
$$

Taking the derivative of

$$
\gamma^{*}=\frac{1-k\left(\delta^{*}\right)}{k\left(\delta^{*}\right)(\beta-1)}
$$

with respect to $\mathcal{L}$ yields

$$
\frac{\partial \gamma^{*}}{\partial \mathcal{L}}=\frac{-\frac{\partial k\left(\delta^{*}\right)}{\partial \mathcal{L}} k\left(\delta^{*}\right)(\beta-1)-\left(1-k\left(\delta^{*}\right)\right) \frac{\partial k\left(\delta^{*}\right)}{\partial \mathcal{L}}(\beta-1)}{\left(k\left(\delta^{*}\right)(\beta-1)\right)^{2}}
$$

Hence, $\frac{\partial \delta^{*}}{\partial \mathcal{L}}<0$ implies $\frac{\partial \gamma^{*}}{\partial \mathcal{L}}>0$.

Lastly, note that

$$
\frac{\partial \tau\left(\delta^{*}\right)}{\partial q}=-\frac{2 \frac{\partial \delta^{*}}{\partial q}}{\delta^{*}}\left(\frac{\mathcal{L}}{\beta}-p_{m}\right)
$$

As a result,

$$
\frac{\partial k\left(\delta^{*}\right)}{\partial q}=\frac{\partial \delta^{*}}{\partial q} \int_{\tau\left(\delta^{*}\right)}^{1}\left(p-p_{m}\right) f(p) d p+\left(\delta^{*} \tau\left(\delta^{*}\right)+\left(1-\delta^{*}\right) p_{m}\right) f\left(\tau\left(\delta^{*}\right)\right) \frac{2 \frac{\partial \delta^{*}}{\partial q}}{\delta^{*}}\left(\frac{\mathcal{L}}{\beta}-p_{m}\right)
$$

Taking the derivative of

$$
\gamma^{*}=\frac{1-k\left(\delta^{*}\right)}{k\left(\delta^{*}\right)(\beta-1)}
$$

with respect to $q$ yields

$$
\frac{\partial \gamma^{*}}{\partial q}=\frac{\left.-\frac{\partial k\left(\delta^{*}\right)}{\partial q} k\left(\delta^{*}\right)(\beta-1)-\left(1-k\left(\delta^{*}\right)\right) \frac{\partial k\left(\delta^{*}\right)}{\partial q}(\beta-1)\right)}{\left(k\left(\delta^{*}\right)(\beta-1)\right)^{2}}
$$

Hence, for $c$ sufficiently small, $\frac{\partial \delta^{*}}{\partial q}<0$ implies $\frac{\partial \gamma^{*}}{\partial q}>0$.

Finally, let us study the comparative statics with respect to $\beta$. Note that

$$
\frac{\partial \tau\left(\delta^{*}\right)}{\partial \beta}=-\frac{\mathcal{L}}{\delta^{*} \beta^{2}}-\frac{2 \frac{\partial \delta^{*}}{\partial \beta}}{\delta^{*}}\left(\frac{\mathcal{L}}{\beta}-p_{m}\right)
$$


As a result,

$$
\frac{\partial k\left(\delta^{*}\right)}{\partial \beta}=\frac{\partial \delta^{*}}{\partial \beta} \int_{\tau\left(\delta^{*}\right)}^{1}\left(p-p_{m}\right) f(p) d p+\left(\delta^{*} \tau\left(\delta^{*}\right)+\left(1-\delta^{*}\right) p_{m}\right) f\left(\tau\left(\delta^{*}\right)\right)\left(\frac{\mathcal{L}}{\delta^{*} \beta^{2}}+\frac{2 \frac{\partial \delta^{*}}{\partial \beta}}{\delta^{*}}\left(\frac{\mathcal{L}}{\beta}-p_{m}\right)\right) .
$$

Taking the derivative of

$$
\gamma^{*}=\frac{1-k\left(\delta^{*}\right)}{k\left(\delta^{*}\right)(\beta-1)}
$$

with respect to $\beta$ yields

$$
\frac{\partial \gamma^{*}}{\partial \beta}=\frac{-\frac{\partial k\left(\delta^{*}\right)}{\partial \beta} k\left(\delta^{*}\right)(\beta-1)-\left(1-k\left(\delta^{*}\right)\right)\left(k\left(\delta^{*}\right)+\frac{\partial k\left(\delta^{*}\right)}{\partial \beta}(\beta-1)\right)}{\left(k\left(\delta^{*}\right)(\beta-1)\right)^{2}},
$$

which is equivalent to

$$
\frac{\partial \gamma^{*}}{\partial \beta}=-\frac{\left(1-k\left(\delta^{*}\right)\right) k\left(\delta^{*}\right)+(\beta-1) \frac{\partial k\left(\delta^{*}\right)}{\partial \beta}}{(k(\delta)(\beta-1))^{2}} .
$$

Hence, $\frac{\partial \delta^{*}}{\partial \beta}>0$ implies that $\frac{\partial k\left(\delta^{*}\right)}{\partial \beta}>0$, which implies that $\frac{\partial \gamma^{*}}{\partial \beta}<0$. Further, we know that the leverage is given by

$$
\frac{1}{\gamma^{*} \beta-\left(\gamma^{*}-1\right)}=k\left(\delta^{*}\right)
$$

Taking the derivative of the left-hand side with respect to beta yields

$$
E \frac{-\gamma^{*}-(\beta-1) \frac{\partial \gamma^{*}}{\partial \beta}}{\left(\gamma^{*} \beta-\left(\gamma^{*}-E\right)\right)^{2}}
$$

Further, $\frac{\partial \gamma^{*}}{\partial \beta}>0$ implies that the left-hand side of (6.11) decreases in $\beta$, which in turn implies that $k\left(\delta^{*}\right)$ decreases in $\beta$, which is equivalent to

$$
\begin{aligned}
& \frac{\partial k\left(\delta^{*}\right)}{\partial \beta}=\frac{\partial \delta^{*}}{\partial \beta} \int_{\tau\left(\delta^{*}\right)}^{1}\left(p-p_{m}\right) \\
& \quad f(p) d p \\
& \quad+\left(\delta^{*} \tau\left(\delta^{*}\right)+\left(1-\delta^{*}\right) p_{m}\right) f\left(\tau\left(\delta^{*}\right)\right)\left(\frac{\mathcal{L}}{\delta^{*} \beta^{2}}+\frac{2 \frac{\partial \delta^{*}}{\partial \beta}}{\delta^{*}}\left(\frac{\mathcal{L}}{\beta}-p_{m}\right)\right)<0
\end{aligned}
$$

As a result, $\delta^{*}$ decreases in $\beta$.

\section{Proof of corollary 3:}

Assume that the regulator chooses different capital requirements and different reporting systems: $\left(\gamma_{G}, \delta_{G}\right)$ and $\left(\gamma_{B}, \delta_{B}\right)$ In equilibrium, a good bank voluntary chooses the policies $\left(\gamma_{G}, \delta_{G}\right)$ whereas a bad bank chooses the policies $\left(\gamma_{B}, \delta_{B}\right)$. Our objective is to prove that the regulator cannot increase the 
expected surplus compared to a setting in which the regulator chooses the same policies $(\gamma, \delta)$ for both types of banks.

First, the expected surplus can be written as

$$
\begin{aligned}
\max _{\gamma_{G}, \gamma_{B}, \delta_{G}, \delta_{B}} \Sigma\left(\gamma_{G}, \gamma_{B}, \delta_{G}, \delta_{B}\right)= & \frac{1}{2}\left(q \alpha+(1-q)\left(F\left(\tau_{G}\left(\delta_{G}\right)\right) \mathcal{L}+k_{G}\left(\delta_{G}\right) \beta\right)-1\right) \gamma_{G} E \\
& +\frac{1}{2}\left(F\left(\tau_{B}\left(\delta_{B}\right)\right) \mathcal{L}+k_{B}\left(\delta_{B}\right) \beta-1\right) \gamma_{B} E
\end{aligned}
$$

where the restructuring thresholds $\tau_{G}\left(\delta_{G}\right)$ and $\tau_{B}\left(\delta_{B}\right)$ are defined as follows

$$
\tau_{G}\left(\delta_{G}\right) \equiv \begin{cases}1 & \text { if } 0 \leq \delta_{G}<\frac{\frac{\mathcal{L}}{\beta}-p_{m}}{1-p_{m}} \\ \frac{1}{\delta_{G}}\left(\frac{\mathcal{L}}{\beta}-\left(1-\delta_{G}\right) p_{m}\right) & \text { otherwise }\end{cases}
$$

and

$$
\tau_{B}\left(\delta_{B}\right) \equiv \begin{cases}1 & \text { if } 0 \leq \delta_{B}<\frac{\frac{\mathcal{L}}{\beta}-p_{m}}{1-p_{m}} \\ \frac{1}{\delta_{B}}\left(\frac{\mathcal{L}}{\beta}-\left(1-\delta_{B}\right) p_{m}\right) & \text { otherwise }\end{cases}
$$

Second, the regulator faces the following four constraints. A good bank chooses the policy $\left(\gamma_{G}, \delta_{G}\right)$ only if

$$
\begin{aligned}
q\left(\gamma_{G} \alpha-\left(\gamma_{G}-1\right)\right)+(1-q) k_{G}\left(\delta_{G}\right)\left(\gamma_{G} \beta-\left(\gamma_{G}-1\right)\right) & \\
& \geq q\left(\gamma_{B} \alpha-\left(\gamma_{B}-1\right)\right)+(1-q) k_{B}\left(\delta_{B}\right)\left(\gamma_{B} \beta-\left(\gamma_{B}-1\right)\right) .
\end{aligned}
$$

Similarly, a bad bank chooses the policy $\left(\gamma_{B}, \delta_{B}\right)$ only if

$$
k_{B}\left(\delta_{B}\right)\left(\gamma_{B} \beta-\left(\gamma_{B}-1\right)\right) \geq k_{G}\left(\delta_{G}\right)\left(\gamma_{G} \beta-\left(\gamma_{G}-1\right)\right)
$$

Further, $\left(\gamma_{G}, \delta_{G}\right)$ and $\left(\gamma_{B}, \delta_{B}\right)$ should be such that the good bank originates a loan portfolio whereas the bad bank may or may not originate a loan portfolio, i.e., such that

$$
q\left(\gamma_{G} \alpha-\left(\gamma_{G}-1\right)\right)+(1-q) k_{G}\left(\delta_{G}\right)\left(\gamma_{G} \beta-\left(\gamma_{G}-1\right)\right)>1
$$

and

$$
k_{B}\left(\delta_{B}\right)\left(\gamma_{B} \beta-\left(\gamma_{B}-1\right)\right) \geq 1 .
$$


First note that the two constraints (6.16) and (6.17) imply that $q\left(\gamma_{G} \alpha-\left(\gamma_{G}-1\right)\right) \geq q\left(\gamma_{B} \alpha-\left(\gamma_{B}-1\right)\right)$, which implies that $\gamma_{G} \geq \gamma_{B}$. Further, given precisions $\delta_{G}$ and $\delta_{B}$, the regulator's objective function decreases in $\gamma_{B}$ and increases in $\gamma_{G}$. As a result, the two constraints (6.17) and (6.19) are binding. The maximization problem is then the same as in the baseline model with the same policies for both types of banks. $\square$

Proof of lemma 5: In equilibrium, the incentive constraint of the bank binds:

$$
\gamma=\frac{1-k(\delta)}{k(\delta)(\beta-1)-(\alpha-1)}
$$

Further, the restructuring threshold is defined as

$$
\tau(\delta) \equiv \begin{cases}1 & \text { if } 0 \leq \delta<\frac{\frac{\mathcal{L}+\mathcal{K}}{\beta+\mathcal{K}}-p_{m}}{1-p_{m}} \\ \frac{1}{\delta}\left(\frac{\mathcal{L}+\mathcal{K}}{\beta+\mathcal{K}}-(1-\delta) p_{m}\right) & \text { otherwise }\end{cases}
$$

Substituting $\gamma$ into the objective of the regulator, we can write $(P)$ as

$$
\max _{\delta} \Sigma_{b}(\delta) \equiv(q \alpha+(1-q)(F(\tau(\delta)) \mathcal{L}+k(\delta) \beta-(1-k(\delta)) \mathcal{K})-1) \frac{1-k(\delta)}{k(\delta)(\beta-1)-(\alpha-1)} E
$$

Taking the first-order condition with respect to $\delta$, we get

$$
\begin{aligned}
&(k(\delta)(\beta-1)-(\alpha-1))\left(-k^{\prime}(\delta)(q \alpha+(1-q)(F(\tau(\delta)) \mathcal{L}+k(\delta) \beta-(1-k(\delta)) \mathcal{K})-1)\right. \\
&\left.+(1-k(\delta))(1-q)\left(f(\tau(\delta)) \mathcal{L} \tau^{\prime}(\delta)+k^{\prime}(\delta)(\beta+\mathcal{K})\right)\right) \\
&-(\beta-1) k^{\prime}(\delta)(q \alpha+(1-q)(F(\tau(\delta)) \mathcal{L}+k(\delta) \beta-(1-k(\delta)) \mathcal{K})-1)(1-k(\delta))=0,
\end{aligned}
$$

which is equivalent to

$$
\begin{aligned}
-(q \alpha+(1-q) & (F(\tau(\delta)) \mathcal{L}+k(\delta) \beta-(1-k(\delta) \mathcal{K})-1)(\beta-\alpha) k^{\prime}(\delta) \\
+ & (k(\delta)(\beta-1)-(\alpha-1))(1-k(\delta))(1-q)\left(f(\tau(\delta)) \tau^{\prime}(\delta) \mathcal{L}+k^{\prime}(\delta)(\beta+\mathcal{K})\right)=0 .
\end{aligned}
$$

Defining the function $H(\delta)$ as the left-hand side of (6.21), we can rewrite the first-order condition as $H(\delta)=0$.

Let us derive the conditions under which $H(1)<0$, or equivalently, the conditions under which $\delta^{*}<1$. 
Evaluating the first-order condition at $\delta=1$, we get

$$
\begin{aligned}
H(1)= & -\left(q \alpha+(1-q)\left(F\left(\frac{\mathcal{L}+\mathcal{K}}{\beta+\mathcal{K}}\right) \mathcal{L}+k(1) \beta-(1-k(1)) \mathcal{K}\right)-1\right) \\
& \times(\beta-\alpha)\left(\int_{\frac{\mathcal{L}+\mathcal{K}}{\beta+\mathcal{K}}}^{1}\left(p-p_{m}\right) f(p) d p-\frac{\mathcal{L}+\mathcal{K}}{\beta+\mathcal{K}} \tau^{\prime}(1) f\left(\frac{\mathcal{L}+\mathcal{K}}{\beta+\mathcal{K}}\right)\right) \\
& +(k(1)(\beta-1)-(\alpha-1))(1-k(1))(1-q)\left(\int_{\frac{\mathcal{L}+\mathcal{K}}{\beta+\mathcal{K}}}^{1}\left(p-p_{m}\right) f(p) d p(\beta+\mathcal{K})-\tau^{\prime}(1) f\left(\frac{\mathcal{L}+\mathcal{K}}{\beta+\mathcal{K}}\right) \mathcal{K}\right) \\
= & -\left(q \alpha+(1-q)\left(F\left(\frac{\mathcal{L}+\mathcal{K}}{\beta+\mathcal{K}}\right) \mathcal{L}+k(1) \beta-(1-k(1)) \mathcal{K}\right)-1\right) \\
& \times(\beta-\alpha)\left(\int_{\frac{\mathcal{L}+\mathcal{K}}{\beta+\mathcal{K}}}^{1}\left(p-p_{m}\right) f(p) d p-\frac{\mathcal{L}+\mathcal{K}}{\beta+\mathcal{K}}\left(p_{m}-\frac{\mathcal{L}+\mathcal{K}}{\beta+\mathcal{K}}\right) f\left(\frac{\mathcal{L}+\mathcal{K}}{\beta+\mathcal{K}}\right)\right) \\
& +(k(1)(\beta-1)-(\alpha-1))(1-k(1))(1-q)\left(\int_{\frac{\mathcal{L}+\mathcal{K}}{\beta+\mathcal{K}}}^{1}\left(p-p_{m}\right) f(p) d p(\beta+\mathcal{K})\right. \\
& \left.-\left(p_{m}-\frac{\mathcal{L}+\mathcal{K}}{\beta+\mathcal{K}}\right) f\left(\frac{\mathcal{L}+\mathcal{K}}{\beta+\mathcal{K}}\right) \mathcal{K}\right),
\end{aligned}
$$

where the equalities follow from the fact that $k^{\prime}(\delta)=\int_{\tau(\delta)}^{1}\left(p-p_{m}\right) f(p) d p-\delta \tau^{\prime}(\delta) \tau(\delta) f(\tau(\delta))-(1-$ $\delta) \tau^{\prime}(\delta) p_{m} f(\tau(\delta))$ and $\tau(1)=\frac{\mathcal{L}+\mathcal{K}}{\beta+\mathcal{K}}$.

Full precision $\delta^{*}=1$ cannot be a solution to $(P)$ if $H(1)<0$. If $k(1)(\beta-1)-(\alpha-1)$ is sufficiently small, then $H(1)<0$. Further, it is readily seen that $H(1)$ is decreasing in $q$. As a result, if $q$ is sufficiently large, then $H(1)<0$.

\section{Proof of corollary 4:}

As in the baseline model, we assume that the program is convex so that the comparative statics are 
well-defined. We have

$$
\begin{aligned}
& \frac{\partial H\left(\delta^{*}\right)}{\partial \mathcal{K}}=-(\beta-\alpha)\left(q \alpha+(1-q)\left(F\left(\tau\left(\delta^{*}\right)\right) \mathcal{L}+k\left(\delta^{*}\right) \beta-\left(1-k\left(\delta^{*}\right)\right) \mathcal{K}\right)-1\right) \\
& \times\left[-\left(\tau\left(\delta^{*}\right)-p_{m}\right) f\left(\tau\left(\delta^{*}\right)\right) \frac{\beta-\mathcal{L}}{\delta^{*}(\beta+\mathcal{K})^{2}}+\frac{1}{\left(\delta^{*}\right)^{2}}\left(\frac{f^{\prime}\left(\tau\left(\delta^{*}\right)\right)(\beta-\mathcal{L})}{\delta^{*}(\beta+\mathcal{K})^{2}} \frac{\mathcal{L}+\mathcal{K}}{\beta+\mathcal{K}}\left(\frac{\mathcal{L}+\mathcal{K}}{\beta+\mathcal{K}}-p_{m}\right)\right.\right. \\
& \left.\left.+\frac{f\left(\tau\left(\delta^{*}\right)\right)(\beta-\mathcal{L})}{(\beta+\mathcal{K})^{2}}\left(2 \frac{\mathcal{L}+\mathcal{K}}{\beta+\mathcal{K}}-p_{m}\right)\right)\right] \\
& -(\beta-\alpha)\left(\int_{\tau\left(\delta^{*}\right)}^{1}\left(p-p_{m}\right) f(p) d p+\frac{f\left(\tau\left(\delta^{*}\right)\right)}{\left(\delta^{*}\right)^{2}} \frac{\mathcal{L}+\mathcal{K}}{\beta+\mathcal{K}}\left(\frac{\mathcal{L}+\mathcal{K}}{\beta+\mathcal{K}}-p_{m}\right)\right) \\
& \times\left[(1-q) f\left(\tau\left(\delta^{*}\right)\right) \mathcal{L} \frac{\beta-\mathcal{L}}{\delta^{*}(\beta+\mathcal{K})^{2}}-(1-q)(\beta+\mathcal{K}) \tau\left(\delta^{*}\right) \frac{f\left(\tau\left(\delta^{*}\right)\right)(\beta-\mathcal{L})}{\delta^{*}(\beta+\mathcal{K})^{2}}-(1-q)\left(1-k\left(\delta^{*}\right)\right)\right] \\
& -\tau\left(\delta^{*}\right) \frac{f\left(\tau\left(\delta^{*}\right)\right)(\beta-\mathcal{L})}{\delta^{*}(\beta+\mathcal{K})^{2}}\left((\beta-1)+(\alpha-1)-2 k\left(\delta^{*}\right)(\beta-1)\right)(1-q)\left(\int_{\tau\left(\delta^{*}\right)}^{1}\left(p-p_{m}\right) f(p) d p(\beta+\mathcal{K})\right. \\
& \left.-\left(p_{m}-\frac{\mathcal{L}+\mathcal{K}}{\beta+\mathcal{K}}\right) \frac{f\left(\tau\left(\delta^{*}\right)\right)}{\left(\delta^{*}\right)^{2}} \mathcal{K}\right) \\
& -\left(k\left(\delta^{*}\right)(\beta-1)-(\alpha-1)\right)\left(1-k\left(\delta^{*}\right)\right)(1-q)\left(\left(\tau\left(\delta^{*}\right)-p_{m}\right) \frac{f\left(\tau\left(\delta^{*}\right)\right)(\beta-\mathcal{L})}{\delta^{*}(\beta+\mathcal{K})}-\int_{\tau\left(\delta^{*}\right)}^{1}\left(p-p_{m}\right) f(p) d p\right. \\
& \left.+\left(p_{m}-\frac{\mathcal{L}+\mathcal{K}}{\beta+\mathcal{K}}\right) f^{\prime}\left(\tau\left(\delta^{*}\right)\right) \frac{\mathcal{K}(\beta-\mathcal{L})}{\left(\delta^{*}\right)^{3}(\beta+\mathcal{K})^{2}}-\frac{(\beta-\mathcal{L}) f\left(\tau\left(\delta^{*}\right)\right) \mathcal{K}}{\left(\delta^{*}\right)^{2}(\beta+\mathcal{K})^{2}}+\left(p_{m}-\frac{\mathcal{L}+\mathcal{K}}{\beta+\mathcal{K}}\right) \frac{f\left(\tau\left(\delta^{*}\right)\right)}{\left(\delta^{*}\right)^{2}}\right) .
\end{aligned}
$$

As $q$ approaches 1 , the sign of $\frac{\partial H\left(\delta^{*}\right)}{\partial \mathcal{K}}$ is given by the sign of

$$
\begin{aligned}
\frac{\partial H\left(\delta^{*}\right)}{\partial \mathcal{K}}= & -(\beta-\alpha)\left(q \alpha+(1-q)\left(F\left(\tau\left(\delta^{*}\right)\right) \mathcal{L}+k\left(\delta^{*}\right) \beta-\left(1-k\left(\delta^{*}\right)\right) \mathcal{K}\right)-1\right) \\
& \times\left[-\left(\tau\left(\delta^{*}\right)-p_{m}\right) f\left(\tau\left(\delta^{*}\right)\right) \frac{\beta-\mathcal{L}}{\delta^{*}(\beta+\mathcal{K})^{2}}+\frac{1}{\left(\delta^{*}\right)^{2}}\left(\frac{f^{\prime}\left(\tau\left(\delta^{*}\right)\right)(\beta-\mathcal{L})}{\delta^{*}(\beta+\mathcal{K})^{2}} \frac{\mathcal{L}+\mathcal{K}}{\beta+\mathcal{K}}\left(\frac{\mathcal{L}+\mathcal{K}}{\beta+\mathcal{K}}-p_{m}\right)\right.\right. \\
& \left.\left.+\frac{f\left(\tau\left(\delta^{*}\right)\right)(\beta-\mathcal{L})}{(\beta+\mathcal{K})^{2}}\left(2 \frac{\mathcal{L}+\mathcal{K}}{\beta+\mathcal{K}}-p_{m}\right)\right)\right]
\end{aligned}
$$

As a result, if $q$ is sufficiently large and $f^{\prime}\left(\tau\left(\delta^{*}\right)\right) \geq 0$ or $f^{\prime}\left(\tau\left(\delta^{*}\right)\right)$ sufficiently close to 0 , then $\frac{\partial H\left(\delta^{*}\right)}{\partial \mathcal{K}}<0$.

Further, note that

$$
\frac{\partial \tau\left(\delta^{*}\right)}{\partial \mathcal{K}}=\frac{\beta-\mathcal{L}}{\delta^{*}(\beta+\mathcal{K})^{2}}-\frac{\frac{\partial \delta^{*}}{\partial \mathcal{K}}}{\left(\delta^{*}\right)^{2}}\left(\frac{\mathcal{L}+\mathcal{K}}{\beta+\mathcal{K}}-p_{m}\right)
$$

As a result,

$$
\frac{\partial k\left(\delta^{*}\right)}{\partial \mathcal{K}}=\frac{\partial \delta^{*}}{\partial \mathcal{K}} \int_{\tau\left(\delta^{*}\right)}^{1}\left(p-p_{m}\right) f(p) d p+\left(\delta^{*} \tau\left(\delta^{*}\right)+\left(1-\delta^{*}\right) p_{m}\right) f\left(\tau\left(\delta^{*}\right)\right)\left(-\frac{\beta-\mathcal{L}}{\delta^{*}(\beta+\mathcal{K})^{2}}+\frac{\frac{\partial \delta^{*}}{\partial \mathcal{K}}}{\left(\delta^{*}\right)^{2}}\left(\frac{\mathcal{L}+\mathcal{K}}{\beta+\mathcal{K}}-p_{m}\right)\right)
$$

Moreover, taking the derivative of

$$
\gamma^{*}=\frac{1-k\left(\delta^{*}\right)}{k\left(\delta^{*}\right)(\beta-1)-(\alpha-1)}
$$


with respect to $\mathcal{K}$ yields

$$
\frac{\partial \gamma^{*}}{\partial \mathcal{K}}=\frac{\left.-\frac{\partial k\left(\delta^{*}\right)}{\partial \mathcal{K}}\left(k\left(\delta^{*}\right)(\beta-1)-(\alpha-1)\right)-\left(1-k\left(\delta^{*}\right)\right) \frac{\partial k\left(\delta^{*}\right)}{\partial \mathcal{K}}(\beta-1)\right)}{\left(k\left(\delta^{*}\right)(\beta-1)-(\alpha-1)\right)^{2}}
$$

As a result, if $\frac{\partial \delta^{*}}{\partial \mathcal{K}}<0$, then $\frac{\partial k\left(\delta^{*}\right)}{\partial \mathcal{K}}<0$, which implies that $\frac{\partial \gamma^{*}}{\partial \mathcal{K}}>$

\section{Proof of lemma 5:}

The regulator's maximization problem is equivalent to choosing a probability of restructuring $\kappa($.$) as$ a function of $p$ and a capital requirement $\gamma$ to maximize the expected surplus:

$$
\max _{\gamma, \kappa(.) \in[0,1]} \int_{0}^{1}((q \alpha+(1-q)(\kappa(p) \mathcal{L}+(1-\kappa(p)) p \beta)-1) \gamma E) f(p) d p
$$

s.t.

$$
\begin{aligned}
\frac{\mathcal{L}}{\beta} \int_{0}^{1} \kappa(p) f(p) d p-\int_{0}^{1} p \kappa(p) f(p) d p & \geq 0 \\
\int_{0}^{1} p(1-\kappa(p)) f(p) d p-\frac{\mathcal{L}}{\beta} \int_{0}^{1}(1-\kappa(p)) f(p) d p & \geq 0 \\
\gamma \alpha-(\gamma-1)-\int_{0}^{1} p(1-\kappa(p)) f(p) d p(\gamma \beta-(\gamma-1)) & \geq 0,
\end{aligned}
$$

where the constraints (6.22) and (6.23) ensure that the regulatory action is ex-post efficient given the signal $s$, and (6.24) is the incentive constraint. We show that the restructuring policy must take the form of a threshold above which the bank is continued.

We define the Lagrangian similarly with the lagrange multipliers $\mu_{0}, \mu_{a}$ and $\mu_{b}$, associated to constraints $(6.24),(6.22)$ and $(6.23)$ respectively. Differentiating with respect to $\kappa(p)$,

$$
\frac{\partial L}{\partial \kappa(p)}=p\left(\mu_{0}(\gamma \beta-(\gamma-1))-(1-q) \beta \gamma E-\mu_{a}-\mu_{b}\right)+\left((1-q) \gamma E+\frac{\mu_{a}+\mu_{b}}{\beta}\right) \mathcal{L}
$$

Noting that this function is linear in $p$ and positive at $p=0$, we know that $\kappa(p)=1$ if and only if $p \leq \tau$, where $\tau$ is a threshold in $[0,1]$.

Case 1. Suppose that $\mu_{0}=0$. Then, the solution $\mu_{a}=\mu_{b}=0$ and $\tau=\mathcal{L} / \beta$ maximizes the Lagrangian and satisfies the constraints associated to multipliers $\mu_{a}$ and $\mu_{b}$. But, then, it is desirable to set $\gamma=\infty$, which contradicts A0.

Case 2. Suppose that $\mu_{0}>0$. Writing the incentive constraint explicitly after reinjecting $\kappa(p)=1$ if and only if $p<\tau$,

$$
\gamma \alpha-(\gamma-1)-\int_{\tau}^{1} p f(p) d p(\gamma \beta-(\gamma-1)) \geq 0
$$


The left-hand side is increasing in $\tau$ and the objective function is increasing in $\tau$ when $\tau \in(0, \mathcal{L} / \beta)$, hence, it must be that, if the (IC) is binding, $\tau \geq \mathcal{L} / \beta$.

\section{Proof of proposition 5:}

Suppose that the efficient restructuring threshold is such that the incentive constraint does not bind . Then, it is optimal for the regulator to set the threshold $\tau=\mathcal{L} / \beta$. This threshold satisfies the constraints (6.22) and (6.23). The expected utility of the regulator is

$$
\left(q \alpha+(1-q) F\left(\frac{\mathcal{L}}{\beta}\right) \mathcal{L}+(1-q) \int_{\frac{\mathcal{L}}{\beta}}^{1} p \beta f(p) d p-1\right) A
$$

This last expression is positive from A0. But then, it is desirable to set $\gamma=\infty$. This is a contradiction with A0. Hence, the incentive constraint binds in equilibrium.

Let us denote

$$
H(\tau)=\Sigma^{\prime}(\tau)\left(1-\int_{\tau}^{1} p f(p) d p-\left(\alpha-\beta \int_{\tau}^{1} p f(p) d p\right)\right)^{2} / E
$$

Taking the first-order condition of the optimization problem,

$$
\begin{aligned}
H(\tau)=(q \alpha+ & \left.(1-q)\left(\mathcal{L} F(\tau)+\beta \int_{\tau}^{1} p f(p) d p\right)-1\right)(\beta-\alpha) \tau \\
& +(1-q)(\mathcal{L}-\tau \beta)\left(1-\int_{\tau}^{1} p f(p) d p\right)\left(1-\int_{\tau}^{1} p f(p) d p-\left(\alpha-\beta \int_{\tau}^{1} p f(p) d p\right)\right)=0
\end{aligned}
$$

Evaluating this expression at $\tau=\mathcal{L} / \beta$,

$$
H\left(\frac{\mathcal{L}}{\beta}\right)=\left(q \alpha+(1-q)\left(\mathcal{L} F\left(\frac{\mathcal{L}}{\beta}\right)+\beta \int_{\frac{\mathcal{L}}{\beta}}^{1} p f(p) d p\right)-1\right)(\beta-\alpha) \frac{\mathcal{L}}{\beta}>0
$$

so that the threshold $\mathcal{L} / \beta$ is never the ex-ante optimal choice for the regulator. Further, we know that $\tau^{*} \geq \mathcal{L} / \beta$ which implies that the restructuring threshold must be in the set $(\mathcal{L} / \beta, \bar{\tau}] . \square$

Proof of proposition 6: Since the 'if' part is immediate, we prove here the 'only if' part. Differentiating $H$ in $\tau$,

$$
\begin{aligned}
& H^{\prime}(\tau)=\left(q \alpha+(1-q)\left(\mathcal{L} F(\tau)+\beta \int_{\tau}^{1} p f(p) d p\right)-1\right)(\beta-\alpha) \\
& +\tau(\beta-\alpha)(1-q) f(\tau)(\mathcal{L}-\tau \beta)-(1-q) \beta\left(1-\int_{\tau}^{1} p f(p) d p\right)\left(1-\int_{\tau}^{1} p f(p) d p-\left(\alpha-\beta \int_{\tau}^{1} p f(p) d p\right)\right) \\
& +(1-q)(\mathcal{L}-\tau \beta)\left(\tau f(\tau)\left(1-\int_{\tau}^{1} p f(p) d p-\left(\alpha-\beta \int_{\tau}^{1} p f(p) d p\right)\right)+\tau f(\tau)(1-\beta)\left(1-\int_{\tau}^{1} p f(p) d p\right)\right) .
\end{aligned}
$$


To simplify the above equation, let us rewrite equation (6.26) at the optimal threshold $\tau^{*}$ as

$$
\begin{aligned}
\left(q \alpha+(1-q)\left(\mathcal{L} F\left(\tau^{*}\right)\right.\right. & \left.\left.+\beta \int_{\tau^{*}}^{1} p f(p) d p\right)-1\right)(\beta-\alpha) \tau^{*} \\
& =-(1-q)\left(\mathcal{L}-\tau^{*} \beta\right)\left(1-\int_{\tau^{*}}^{1} p f(p) d p\right)\left(1-\int_{\tau^{*}}^{1} p f(p) d p-\left(\alpha-\beta \int_{\tau^{*}}^{1} p f(p) d p\right)\right)
\end{aligned}
$$

Reinjecting this last equality into (6.27) evaluated at $\tau^{*}$ yields

$$
\begin{aligned}
& H^{\prime}\left(\tau^{*}\right)=\tau^{*}(\beta-\alpha)(1-q) f\left(\tau^{*}\right)\left(\mathcal{L}-\tau^{*} \beta\right) \\
& \quad-(1-q) \frac{\mathcal{L}}{\tau^{*}}\left(1-\int_{\tau^{*}}^{1} p f(p) d p\right)\left(1-\int_{\tau^{*}}^{1} p f(p) d p-\left(\alpha-\beta \int_{\tau^{*}}^{1} p f(p) d p\right)\right) \\
& +(1-q)\left(\mathcal{L}-\tau^{*} \beta\right)\left(\tau^{*} f\left(\tau^{*}\right)\left(1-\int_{\tau^{*}}^{1} p f(p) d p-\left(\alpha-\beta \int_{\tau^{*}}^{1} p f(p) d p\right)\right)+\tau^{*} f\left(\tau^{*}\right)(1-\beta)\left(1-\int_{\tau^{*}}^{1} p f(p) d p\right)\right) . \\
& =\underbrace{-\left(2 \tau^{*}(1-q) f\left(\tau^{*}\right)\left(\tau^{*} \beta-\mathcal{L}\right)+(1-q) \frac{\mathcal{L}}{\tau^{*}}\left(1-\int_{\tau^{*}}^{1} p f(p) d p\right)\right)}_{H_{1}} \times \underbrace{\left(\beta \int_{\tau^{*}}^{1} p f(p) d p+1-\int_{\tau^{*}}^{1} p f(p) d p-\alpha\right)}_{H_{2}} .
\end{aligned}
$$

It is immediate to verify that $H_{1}<0$. Next, $H_{2}>0$ can be written as

$$
\frac{\beta-1}{\alpha-1}>\frac{1}{\int_{\tau^{*}}^{1} p f(p) d p}
$$

This last inequality is an implication from the incentive constraint, since we know from the incentive constraint that

$$
\frac{1}{\int_{\tau^{*}}^{1} p f(p) d p}=\frac{\gamma \beta-\gamma+1}{\gamma \alpha-\gamma+1}<\frac{\beta-1}{\alpha-1}
$$

Therefore, we have shown that $\Sigma^{\prime}\left(\tau^{*}\right)<0$. Note that this holds at $\tau^{*}$ as well as at any root of $\Sigma^{\prime}($.$) ,$ implying that $\Sigma^{\prime}($.) can cross zero at most once and from below so that $\Sigma$ has a single peak. Evaluating $\Sigma^{\prime}($.$) at \bar{\tau}$, we know that the peak is located below $\bar{\tau}$ if $\Sigma^{\prime}(\bar{\tau}) \leq 0$ and above $\bar{\tau}$ if $\Sigma^{\prime}(\bar{\tau})>0$. 
We can further rewrite

$$
\begin{aligned}
H(\bar{\tau})= & \left(q \alpha+(1-q)\left(\mathcal{L} F(\bar{\tau})+\beta \int_{\bar{\tau}}^{1} p f(p) d p-1\right)(\beta-\alpha) \bar{\tau}\right. \\
& +(1-q)(\mathcal{L}-\bar{\tau} \beta)\left(1-\int_{\bar{\tau}}^{1} p f(p) d p\right)\left(1-\int_{\bar{\tau}}^{1} p f(p) d p-\left(\alpha-\beta \int_{\bar{\tau}}^{1} p f(p) d p\right)\right) \\
= & (q \alpha+(1-q) \beta \mathbb{E}(s)-1)(\beta-\alpha) \bar{\tau} \\
& +(1-q)(\mathcal{L}-\bar{\tau} \beta)\left(1+\frac{\mathcal{L} F(\bar{\tau})}{\beta}-\mathbb{E}(s)\right)\left(1-\alpha+(\beta-1)\left(\mathbb{E}(s)-\frac{\mathcal{L}}{\beta} F(\bar{\tau})\right)\right),
\end{aligned}
$$

where the last equality is obtained from the definition of $\bar{\tau}$. Thus, $\Sigma^{\prime}(\bar{\tau})>0$ is equivalent to

$$
q>\frac{(1-\beta \mathbb{E}(s))(\beta-\alpha) \bar{\tau}-(\mathcal{L}-\bar{\tau} \beta)\left(1+\frac{\mathcal{L} F(\bar{\tau})}{\beta}-\mathbb{E}(s)\right)\left(1-\alpha+(\beta-1)\left(\mathbb{E}(s)-\frac{\mathcal{L}}{\beta} F(\bar{\tau})\right)\right)}{(\alpha-\beta \mathbb{E}(s))(\beta-\alpha) \bar{\tau}-(\mathcal{L}-\bar{\tau} \beta)\left(1+\frac{\mathcal{L} F(\bar{\tau})}{\beta}-\mathbb{E}(s)\right)\left(1-\alpha+(\beta-1)\left(\mathbb{E}(s)-\frac{\mathcal{L}}{\beta} F(\bar{\tau})\right)\right)}
$$

Therefore, we get condition (4.16) in text. $\square$

Proof of corollary 5: Let us start by deriving the comparative statics in the case $q>\bar{q}$. The threshold $\bar{\tau}$ is defined by (4.15), which is equivalent to $\mathbb{E}(s \mid s \leq \bar{\tau})=\mathcal{L} / \beta$. Obviously, $\bar{\tau}$ does not depend on $\alpha$. Further, as $\mathcal{L}$ increases, $\mathbb{E}(s \mid s \leq \bar{\tau})$ increases, which implies that $\bar{\tau}$ increases. Similarly, as $\beta$ increases, $\mathbb{E}(s \mid s \leq \bar{\tau})$ decreases, which implies that $\bar{\tau}$ decreases.

The optimal capital requirement is given by

$$
\gamma^{*}=\frac{1-\int_{\bar{\tau}}^{1} p f(p) d p}{1-\int_{\bar{\tau}}^{1} p f(p) d p-\left(\alpha-\beta \int_{\bar{\tau}}^{1} p f(p) d p\right)}
$$

Therefore,

$$
\frac{\partial \gamma^{*}}{\partial \bar{\tau}}=\frac{\bar{\tau} f(\bar{\tau})\left(1-\int_{\bar{\tau}}^{1} p f(p) d p-\left(\alpha-\beta \int_{\bar{\tau}}^{1} p f(p) d p\right)\right)+\left(1-\int_{\bar{\tau}}^{1} p f(p) d p\right)(\beta-1) \bar{\tau} f(\bar{\tau})}{\left(1-\int_{\bar{\tau}}^{1} p f(p) d p-\left(\alpha-\beta \int_{\bar{\tau}}^{1} p f(p) d p\right)\right)^{2}}>0 .
$$

As a result,

$$
\frac{\partial \gamma^{*}}{\partial \mathcal{L}}=\frac{\partial \bar{\tau}}{\partial \mathcal{L}} \frac{\bar{\tau} f(\bar{\tau})\left(1-\int_{\bar{\tau}}^{1} p f(p) d p-\left(\alpha-\beta \int_{\bar{\tau}}^{1} p f(p) d p\right)\right)+\left(1-\int_{\bar{\tau}}^{1} p f(p) d p\right)(\beta-1) \bar{\tau} f(\bar{\tau})}{\left(1-\int_{\bar{\tau}}^{1} p f(p) d p-\left(\alpha-\beta \int_{\bar{\tau}}^{1} p f(p) d p\right)\right)^{2}}>0
$$

and

$$
\frac{\partial \gamma^{*}}{\partial \alpha}=\frac{1-\int_{\bar{\tau}}^{1} p f(p) d p}{\left(1-\int_{\bar{\tau}}^{1} p f(p) d p-\left(\alpha-\beta \int_{\bar{\tau}}^{1} p f(p) d p\right)\right)^{2}}>0
$$


Finally, $\bar{\tau}$ decreases in $\beta$. Hence,

$$
\begin{aligned}
& \frac{\partial \gamma^{*}}{\partial \beta}=\frac{\frac{\partial \bar{\tau}}{\partial \beta} f(\bar{\tau})\left(1-\int_{\bar{\tau}}^{1} p f(p) d p-\left(\alpha-\beta \int_{\bar{\tau}}^{1} p f(p) d p\right)\right)}{\left(1-\int_{\bar{\tau}}^{1} p f(p) d p-\left(\alpha-\beta \int_{\bar{\tau}}^{1} p f(p) d p\right)\right)^{2}} \\
&+\frac{\left.\left(1-\int_{\bar{\tau}}^{1} p f(p) d p\right)\left((\beta-1) \bar{\tau} f(\bar{\tau}) \frac{\partial \bar{\tau}}{\partial \beta}-\int_{\bar{\tau}}^{1} p f(p) d p\right)\right)}{\left(1-\int_{\bar{\tau}}^{1} p f(p) d p-\left(\alpha-\beta \int_{\bar{\tau}}^{1} p f(p) d p\right)\right)^{2}}<0,
\end{aligned}
$$

which implies that $\gamma^{*}$ decreases in $\beta$.

Then, we can derive the comparative statics in the case $q \leq \bar{q}$. Let $H(\tau)=\Sigma^{\prime}(\tau)$ be defined as the derivative of the social surplus in $\tau$. We know from Proposition 6 that $\tau^{*}<\bar{\tau}$ and, given that it is a local maximum of $\Sigma, H^{\prime}\left(\tau^{*}\right) \leq 0$. We will assume here that it is regular maximum, $H^{\prime}\left(\tau^{*}\right)<0$ so that the comparative statics are always well-defined. It then follows that the comparative static of $\tau^{*}$ in a variable $X$ has the sign of $\partial H / \partial X$, which we conduct next:

$$
\begin{aligned}
\frac{\partial H\left(\tau^{*}\right)}{\partial \alpha}= & q(\beta-\alpha) \tau^{*}-\tau^{*}\left(q \alpha+(1-q)\left(\mathcal{L} F\left(\tau^{*}\right)+\beta \int_{\tau^{*}}^{1} p f(p) d p\right)-1\right) \\
& -(1-q)\left(\mathcal{L}-\tau^{*} \beta\right)\left(1-\int_{\tau^{*}}^{1} p f(p) d p\right) ; \\
\frac{\partial H\left(\tau^{*}\right)}{\partial \beta}= & (1-q) \int_{\tau^{*}}^{1} p f(p) d p(\beta-\alpha) \tau^{*}+\tau^{*}\left(q \alpha+(1-q)\left(\mathcal{L} F\left(\tau^{*}\right)+\beta \int_{\tau^{*}}^{1} p f(p) d p\right)-1\right) \\
& +(1-q)\left(\mathcal{L}-\tau^{*} \beta\right)\left(1-\int_{\tau^{*}}^{1} p f(p) d p\right) \int_{\tau^{*}}^{1} p f(p) d p \\
& -(1-q) \tau^{*}\left(1-\int_{\tau^{*}}^{1} p f(p) d p\right)\left(1-\alpha+(\beta-1) \int_{\tau^{*}}^{1} p f(p) d p\right) ; \\
\frac{\partial H\left(\tau^{*}\right)}{\partial \mathcal{L}}= & (1-q) F\left(\tau^{*}\right)(\beta-\alpha) \tau^{*} \\
& +(1-q)\left(1-\int_{\tau^{*}}^{1} p f(p) d p\right)\left(1-\alpha+(\beta-1) \int_{\tau^{*}}^{1} p f(p) d p\right)>0 ; \\
\frac{\partial H\left(\tau^{*}\right)}{\partial q}= & \left(\alpha-\mathcal{L} F\left(\tau^{*}\right)-\beta \int_{\tau^{*}}^{1} p f(p) d p\right)(\beta-\alpha) \tau^{*} \\
& -\left(\mathcal{L}-\tau^{*} \beta\right)\left(1-\int_{\tau^{*}}^{1} p f(p) d p\right)\left(1-\alpha+(\beta-1) \int_{\tau^{*}}^{1} p f(p) d p\right) .
\end{aligned}
$$

We know that $H\left(\tau^{*}\right)=0$. Therefore

$$
\begin{aligned}
-\tau^{*}\left(q \alpha+(1-q)\left(\mathcal{L} F\left(\tau^{*}\right)+\beta \int_{\tau^{*}}^{1}\right.\right. & p f(p) d p)-1) \\
& =\frac{1-q}{\beta-\alpha}\left(\mathcal{L}-\tau^{*} \beta\right)\left(1-\int_{\tau^{*}}^{1} p f(p) d p\right)\left(1-\alpha+(\beta-1) \int_{\tau^{*}}^{1} p f(p) d p\right),
\end{aligned}
$$


which implies that $\frac{\partial H}{\partial q}_{\tau=\tau^{*}}=\left(\alpha+\frac{1}{1-q}(q \alpha-1)\right)(\beta-\alpha) \tau^{*}>0$. Similarly, $H\left(\tau^{*}\right)=0$, is equivalent to

$$
\begin{aligned}
-\tau^{*}\left(q \alpha+(1-q)\left(\mathcal{L} F\left(\tau^{*}\right)+\beta \int_{\tau^{*}}^{1}\right.\right. & p f(p) d p)-1) \\
& =\frac{1-q}{\beta-\alpha}\left(\mathcal{L}-\tau^{*} \beta\right)\left(1-\int_{\tau^{*}}^{1} p f(p) d p\right)\left(1-\alpha+(\beta-1) \int_{\tau^{*}}^{1} p f(p) d p\right),
\end{aligned}
$$

which implies that

$$
\begin{aligned}
\frac{\partial H}{\partial \alpha}\left(\tau^{*}\right)=q(\beta-\alpha) \tau^{*}+(1-q)\left(\tau^{*} \beta-\mathcal{L}\right)\left(1-\int_{\tau^{*}}^{1} p f(p) d p\right)\left(1-\frac{1-\alpha+(\beta-1) \int_{\tau^{*}}^{1} p f(p) d p}{\beta-\alpha}\right) \\
\left.=q(\beta-\alpha) \tau^{*}+\frac{1-q}{\beta-\alpha}\left(\tau^{*} \beta-\mathcal{L}\right)\left(1-\int_{\tau^{*}}^{1} p f(p) d p\right)\left(\beta-1-(\beta-1) \int_{\tau^{*}}^{1} p f(p) d p\right)\right)>0 .
\end{aligned}
$$

We can conclude that the optimal threshold $\tau^{*}$ increases in $\alpha$, in $q$ and in $\mathcal{L}$. From the binding incentive constraint, we can also conclude that the optimal capital requirement moves in the same direction than the optimal threshold with respect to $q, \alpha$ and $\mathcal{L}$. More precisely, we know that

$$
\begin{aligned}
\frac{\partial \gamma^{*}}{\partial \mathcal{L}} & =\frac{\partial \tau^{*}}{\partial \mathcal{L}} \frac{\tau^{*} f\left(\tau^{*}\right)\left(1-\int_{\tau^{*}}^{1} p f(p) d p-\left(\alpha-\beta \int_{\tau^{*}}^{1} p f(p) d p\right)\right)+\left(1-\int_{\tau^{*}}^{1} p f(p) d p\right)(\beta-1) \tau^{*} f\left(\tau^{*}\right)}{\left(1-\int_{\tau^{*}}^{1} p f(p) d p-\left(\alpha-\beta \int_{\tau^{*}}^{1} p f(p) d p\right)\right)^{2}}>0, \\
\frac{\partial \gamma^{*}}{\partial q} & =\frac{\partial \tau^{*}}{\partial q} \frac{\tau^{*} f\left(\tau^{*}\right)\left(1-\int_{\tau^{*}}^{1} p f(p) d p-\left(\alpha-\beta \int_{\tau^{*}}^{1} p f(p) d p\right)\right)+\left(1-\int_{\tau^{*}}^{1} p f(p) d p\right)(\beta-1) \tau^{*} f\left(\tau^{*}\right)}{\left(1-\int_{\tau^{*}}^{1} p f(p) d p-\left(\alpha-\beta \int_{\tau^{*}}^{1} p f(p) d p\right)\right)^{2}}>0,
\end{aligned}
$$

and

$$
\begin{aligned}
& \frac{\partial \gamma^{*}}{\partial \alpha}= \\
& \frac{\frac{\partial \tau^{*}}{\partial \alpha} \tau^{*} f\left(\tau^{*}\right)\left(1-\int_{\tau^{*}}^{1} p f(p) d p-\left(\alpha-\beta \int_{\tau^{*}}^{1} p f(p) d p\right)\right)+\left(1-\int_{\tau^{*}}^{1} p f(p) d p\right)\left((\beta-1) \tau^{*} f\left(\tau^{*}\right) \frac{\partial \tau^{*}}{\partial \alpha}+1\right)}{\left(1-\int_{\tau^{*}}^{1} p f(p) d p-\left(\alpha-\beta \int_{\tau^{*}}^{1} p f(p) d p\right)\right)^{2}}>0 .
\end{aligned}
$$

Therefore, the optimal capital requirement $\gamma^{*}$ also increases in $\alpha, q$ and $\mathcal{L}$.

Finally, the comparative statics with respect to $\beta$ is slightly more complicated. Indeed, we know that

$$
\begin{aligned}
& \frac{d H}{d \beta}=(1-q) \int_{\tau}^{1} p f(p) d p(\beta-\alpha) \tau+\tau(q \alpha\left.+(1-q)\left(\mathcal{L} F(\tau)+\beta \int_{\tau}^{1} p f(p) d p\right)-1\right) \\
&-(1-q)(\tau \beta-\mathcal{L})\left(1-\int_{\tau}^{1} p f(p) d p\right) \int_{\tau}^{1} p f(p) d p \\
&-(1-q) \tau\left(1-\int_{\tau}^{1} p f(p) d p\right)\left(1-\alpha+(\beta-1) \int_{\tau}^{1} p f(p) d p\right) .
\end{aligned}
$$

The first two terms are positive whereas the last two terms are negative. There is an ambiguity for the 
following reason. As $\beta$ increases, the risk-shifting problem becomes more severe (first two terms) and the regulator should provide more incentives to the bank to choose the low-risk loan portfolio by increasing the restructuring threshold. On the other hand, as $\beta$ increases, the payoff in case of continuation is increasing (last two terms) and hence, the regulator is willing to reduce the restructuring threshold. Taking the derivative of the incentive constraint with respect to $\beta$, we get

$$
\begin{aligned}
\frac{\partial \gamma^{*}}{\partial \beta}=\frac{\frac{\partial \tau^{*}}{\partial \beta} \tau^{*}}{f\left(\tau^{*}\right)\left(1-\int_{\tau^{*}}^{1} p f(p) d p-\left(\alpha-\beta \int_{\tau^{*}}^{1} p f(p) d p\right)\right)} \\
\left(1-\int_{\tau^{*}}^{1} p f(p) d p-\left(\alpha-\beta \int_{\tau^{*}}^{1} p f(p) d p\right)\right)^{2} \\
-\frac{\left(1-\int_{\tau^{*}}^{1} p f(p) d p\right)\left(-(\beta-1) \frac{\partial \tau^{*}}{\partial \beta} \tau^{*} f\left(\tau^{*}\right)+\int_{\tau^{*}}^{1} p f(p) d p\right)}{\left(1-\int_{\tau^{*}}^{1} p f(p) d p-\left(\alpha-\beta \int_{\tau^{*}}^{1} p f(p) d p\right)\right)^{2}}
\end{aligned}
$$

Hence, $\frac{\partial \tau^{*}}{\partial \beta}<0$ implies that $\frac{\partial \gamma^{*}}{\partial \beta}<0$. Further, we know that the capital requirement is given by

$$
\int_{\tau^{*}}^{1} p f(p) d p=\frac{\gamma^{*} \alpha-\gamma^{*}+1}{\gamma^{*} \beta-\gamma^{*}+1}
$$

Taking the derivative of the right hand side with respect to beta yields

$$
\frac{(\alpha-1) \frac{\partial \gamma^{*}}{\partial \beta}\left(\beta \gamma^{*}-\gamma^{*}+1\right)-\left(\gamma^{*} \alpha-\gamma^{*}+1\right)\left((\beta-1) \frac{\partial \gamma^{*}}{\partial \beta}+\gamma^{*}\right)}{\left(\gamma^{*} \beta-\gamma^{*}+1\right)^{2}}=\frac{(\alpha-\beta) \frac{\partial \gamma^{*}}{\partial \beta}-\left(\gamma^{*} \alpha-\gamma^{*}+1\right) \gamma^{*}}{\left(\gamma^{*} \beta-\gamma^{*}+1\right)^{2}}
$$

Hence, $\frac{\partial \gamma^{*}}{\partial \beta}>0$ implies that the right-hand side of $(6.28)$ decreases in $\beta$, which in turn implies that $\int_{\tau^{*}}^{1} p f(p) d p$ decreases in $\beta$, i.e. $\tau^{*}$ increases in $\beta$.

Proof of corollary 6: In equilibrium, the incentive constraint of the bank binds:

$$
\gamma=\frac{1-\chi k(1)-(1-\chi) k(\delta)}{(\chi k(1)+(1-\chi) k(\delta))(\beta-1)-(\alpha-1)}
$$

Substituting $\gamma$ into the objective of the regulator, we can write $(P)$ as

$$
\begin{aligned}
\max _{\delta}\left(q \alpha+(1-q)\left(\left(\chi F\left(\frac{\mathcal{L}}{\beta}\right)+(1-\chi) F(\tau(\delta))\right) \mathcal{L}+\right.\right. & (\chi k(1)+(1-\chi) k(\delta)) \beta)-1) \\
& \times \frac{1-\chi k(1)-(1-\chi) k(\delta)}{(\chi k(1)+(1-\chi) k(\delta))(\beta-1)-(\alpha-1)} E .
\end{aligned}
$$


Taking the first-order condition with respect to $\delta$, we get

$$
\begin{array}{r}
((\chi k(1)+(1-\chi) k(\delta))(\beta-1)-(\alpha-1))\left(-(1-\chi) k^{\prime}(\delta)\left(q \alpha+(1-q)\left(\left(\chi F\left(\frac{\mathcal{L}}{\beta}\right)+(1-\chi) F(\tau(\delta))\right) \mathcal{L}\right.\right.\right. \\
+(\chi k(1)+(1-\chi) k(\delta)) \beta)-1) \\
\left.+(1-\chi k(1)-(1-\chi) k(\delta))(1-\chi)\left((1-q)\left(f(\tau(\delta)) \mathcal{L} \tau^{\prime}(\delta)+k^{\prime}(\delta) \beta\right)-f(\tau(\delta)) \tau^{\prime}(\delta) c\right)\right) \\
-(1-\chi)(\beta-1) k^{\prime}(\delta)\left(q \alpha+(1-q)\left(\left(\chi F\left(\frac{\mathcal{L}}{\beta}\right)+(1-\chi) F(\tau(\delta))\right) \mathcal{L}+(\chi k(1)+(1-\chi) k(\delta)) \beta\right)-1\right) \\
\times(1-\chi k(1)-(1-\chi) k(\delta))=0
\end{array}
$$

which is equivalent to

$$
\begin{aligned}
-(\beta-\alpha) k^{\prime}(\delta)\left(q \alpha+(1-q)\left(\left(\chi F\left(\frac{\mathcal{L}}{\beta}\right)+(1-\chi) F(\tau(\delta))\right) \mathcal{L}+(\chi k(1)+(1-\chi) k(\delta)) \beta\right)-1\right) \\
+((\chi k(1)+(1-\chi) k(\delta))(\beta-1)-(\alpha-1))(1-\chi k(1)-(1-\chi) k(\delta)) \\
\times\left((1-q)\left(f(\tau(\delta)) \mathcal{L} \tau^{\prime}(\delta)+k^{\prime}(\delta) \beta\right)\right)=0 .
\end{aligned}
$$

Defining the function $H(\delta)$ as the left-hand side of (6.29), we can rewrite the first-order condition as $H(\delta)=0$. Let us derive the conditions under which $H(1)<0$, or equivalently, the conditions under which $\delta^{*}<1$. Evaluating the first-order condition at $\delta=1$, we get

$$
\begin{aligned}
H(1)= & -\left(q \alpha+(1-q)\left(F\left(\frac{\mathcal{L}}{\beta}\right) \mathcal{L}+k(1) \beta\right)-1\right)(\beta-\alpha)\left(\int_{\frac{\mathcal{L}}{\beta}}^{1}\left(p-p_{m}\right) f(p) d p-\frac{\mathcal{L}}{\beta} \tau^{\prime}(1) f\left(\frac{\mathcal{L}}{\beta}\right)\right) \\
& +(k(1)(\beta-1)-(\alpha-1))(1-k(1))(1-q) \int_{\frac{\mathcal{L}}{\beta}}^{1}\left(p-p_{m}\right) f(p) d p \beta
\end{aligned}
$$

Hence, if $k(1)(\beta-1)-(\alpha-1)$ or $\int_{\frac{\mathcal{L}}{\beta}}^{1}\left(p-p_{m}\right) f(p) d p$ is sufficiently small, then $H(1)<1$. Further, it is readily seen that $H(1)$ is decreasing in $q$ if $c$ is small. Hence, we have $H(1)<0$ if $c$ is sufficiently small and $q$ sufficiently large. 
Further, we have

$$
\begin{aligned}
\frac{\partial H\left(\delta^{*}\right)}{\partial \chi}= & -(\beta-\alpha) k^{\prime}\left(\delta^{*}\right)\left((1-q)\left(\left(F\left(\frac{\mathcal{L}}{\beta}\right)-F\left(\tau\left(\delta^{*}\right)\right)\right) \mathcal{L}+\left(k(1)-k\left(\delta^{*}\right)\right) \beta\right)\right) \\
& -\left(\left(\chi k(1)+(1-\chi) k\left(\delta^{*}\right)\right)(\beta-1)-(\alpha-1)\right)\left(k(1)-k\left(\delta^{*}\right)\right)\left((1-q)\left(f\left(\tau\left(\delta^{*}\right)\right) \mathcal{L} \tau^{\prime}\left(\delta^{*}\right)+k^{\prime}\left(\delta^{*}\right) \beta\right)\right) \\
& +\left(k(1)-k\left(\delta^{*}\right)\right)(\beta-1)\left(1-\chi k(1)-(1-\chi) k\left(\delta^{*}\right)\right)\left((1-q)\left(f\left(\tau\left(\delta^{*}\right)\right) \mathcal{L} \tau^{\prime}\left(\delta^{*}\right)+k^{\prime}\left(\delta^{*}\right) \beta\right)\right) \\
= & -(\beta-\alpha) k^{\prime}\left(\delta^{*}\right)(1-q)\left(\left(F\left(\frac{\mathcal{L}}{\beta}\right)-F\left(\tau\left(\delta^{*}\right)\right)\right) \mathcal{L}+\left(k(1)-k\left(\delta^{*}\right)\right) \beta\right) \\
& +\left(\left(1-2\left(\chi k(1)+(1-\chi) k\left(\delta^{*}\right)\right)\right)(\beta-1)+(\alpha-1)\right)\left(k(1)-k\left(\delta^{*}\right)\right)(1-q) \int_{\tau\left(\delta^{*}\right)}^{1}\left(p-p_{m}\right) f(p) d p \beta .
\end{aligned}
$$

Hence, if $(2 \chi k(1)-1)(\beta-1)>\alpha-1$, then $\frac{\partial H\left(\delta^{*}\right)}{\partial \chi}<0$. 


\section{Appendix 2: residual bank equity}

In the main text, we assume that, if the bank gets a risky loan, the bank's shareholders get a zero payoff in case of regulatory intervention. In this appendix, we show that, even if the bank can intervene in a risky loan without transferring it to the regulator, the bank still has zero equity left as long as assumption 4 is satisfied. We briefly discuss the alternative case in which the bank gets a strictly positive payoff in case of intervention in a risky loan. For simplicity, we assume that regulator directly chooses the bank size $A$.

Suppose that the bank can intervene in a risky loan without transferring it to the regulator. In this environment, if the bank has a risky loan and the bank intervenes, the bank obtains $\mathcal{L}$ instead of having to transfer all of its equity to the regulator. For high enough bank size $A$ (to be derived later on), $A \mathcal{L}-(A-E)<0$, i.e., the bank with a risky loan still has zero equity left in case of intervention in a risky loan, so this case is only relevant for environments where banks start with high levels of equity or the agency friction is not very high. For simplicity, we assume that the bank learns perfectly the realization of the probability of default $p$ at $t=1$, but the bank cannot credibly reveal $p$ to the regulator.

At $t=1$, if the bank has originated a low-risk loan portfolio, the bank voluntarily restructures a risky loan if and only if

$$
q(A \alpha-(A-E))+(1-q)(A \mathcal{L}-(A-E))-A c>q(A \alpha-(A-E))+(1-q) p(A \beta-(A-E)),
$$

which is equivalent to

$$
p<\frac{A \mathcal{L}-(A-E)}{A \beta-(A-E)} \leq \frac{\mathcal{L}}{\beta} .
$$

As a result, the bank's restructuring threshold is lower than the regulator's restructuring threshold. The bank therefore never incurs the inspection cost and the optimal intervention is the same as in our baseline model: the regulator restructures a risky loan if and only if $p<\tau(\delta)$, where the threshold $\tau(\delta) \geq \frac{\mathcal{L}}{\beta}$ is the same as in our baseline model.

Given this optimal intervention policy, the bank originates a low-risk loan portfolio at $t=.5$ if and only if

$$
A \alpha-(A-E)>F(\tau(\delta))(A \mathcal{L}-(A-E))+k(\delta)(A \beta-(A-E)) .
$$

As in the baseline model, we rule out the solution with $\delta^{*}=1$ and $A$ arbitrarily large, which implies that the incentive constraint of the bank is binding in equilibrium. The optimal capital requirement $\gamma^{*}$ is then 
given by the binding incentive constraint, i.e,

$$
\frac{A^{*}}{E}=\frac{1-F(\tau(\delta))-k(\delta)}{k(\delta)(\beta-1)-F(\tau(\delta))(1-\mathcal{L})-(\alpha-1)} .
$$

After deriving the optimal capital requirement, let us derive the conditions under which the bank with a risky loan does not get a positive payoff in case of intervention. If $A^{*} \mathcal{L}-\left(A^{*}-E\right) \leq 0$ with $A^{*}$ given by the incentive constraint of the baseline model, i.e.,

$$
\frac{A^{*}}{E}=\frac{1-k\left(\delta^{*}\right)}{k\left(\delta^{*}\right)(\beta-1)-(\alpha-1)},
$$

then the bank with a risky loan always defaults in case of regulatory intervention and we are back to the case analyzed in our baseline model. Note that assumption 4 implies that

$$
\frac{A^{*}}{E} \geq \frac{1-k(1)}{k(1)(\beta-1)-(\alpha-1)} \geq \frac{1}{1-(q \alpha+(1-q) \mathcal{L})}
$$

As a result, under assumption 4, we have

$$
\frac{A^{*}}{E} \geq \frac{1}{1-\mathcal{L}}
$$

which implies that the bank always defaults with regulatory intervention in a risky loan as in our baseline model.

Otherwise, if assumption 4 is not satisfied, then the condition that makes sure that the bank with a risky loan does not default in case of intervention is given by $A^{*} \mathcal{L}-\left(A^{*}-E\right)>0$ with $A^{*}$ given by equation (6.30). This condition is satisfied if we assume that the parameters $(\alpha, \mathcal{L}, q, \beta, c)$ are such that

$$
\frac{1-F\left(\tau\left(\delta^{*}\right)\right)-k\left(\delta^{*}\right)}{k\left(\delta^{*}\right)(\beta-1)-F\left(\tau\left(\delta^{*}\right)\right)(1-\mathcal{L})-(\alpha-1)}<\frac{1}{1-\mathcal{L}} .
$$

